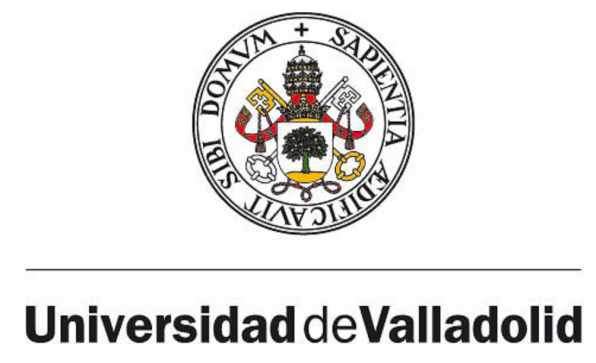

ESCUELA DE INGENIERÍAS INDUSTRIALES

DEPARTAMENTO DE CONSTRUCCIONES ARQUITECTÓNICAS, INGENIERÍA DEL TERRENO Y MECÁNICA DE LOS MEDIOS CONTINUOS Y TEORÍA DE ESTRUCTURAS

TESIS DOCTORAL:

TÉCNICAS NUMÉRICAS DE ANÁLISIS LÍMITE DE PÓRTICOS PLANOS, INCLUYENDO INESTABILIDAD Y COMPORTAMIENTO VIBRATORIO

Presentada por MARIANO CACHO PÉREZ para optar al grado de doctor por la Universidad de Valladolid

Dirigida por:

ANTOLÍN LORENZANA IBÁN

Dr. Ingeniero Industrial 


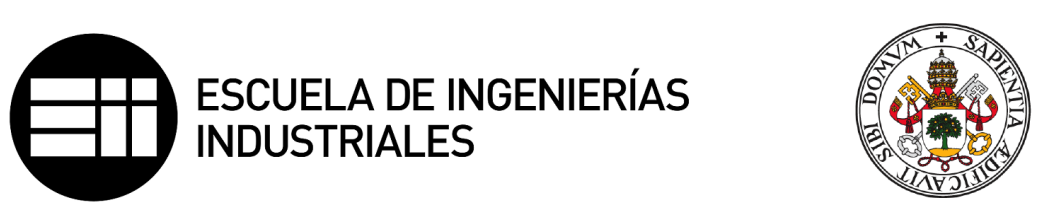

UNIVERSIDAD DE VALLADOLID

DEPARTAMENTO DE CONSTRUCCIONES ARQUITECTÓNICAS, INGENIERÍA DEL TERRENO Y MECÁNICA DE LOS MEDIOS CONTINUOS Y TEORÍA DE ESTRUCTURAS

\title{
TESIS DOCTORAL: \\ TÉCNICAS NUMÉRICAS DE ANÁLISIS LÍMITE DE PÓRTICOS PLANOS, INCLUYENDO INESTABILIDAD Y COMPORTAMIENTO VIBRATORIO
}

\author{
Presentada por D. MARIANO CACHO PÉREZ \\ Ingeniero Industrial de la E.T.S. de Ingenieros Industriales de Valladolid
}

Dirigida por Dr. D. ANTOLÍN LORENZANA IBÁN

Profesor Titular en el Departamento de Construcciones Arquitectónicas, Ingeniería del Terreno y Mecánica de los Medios Continuos y Teoría de Estructuras. Universidad de Valladolid.

Esta Tesis Doctoral fue leída en la Escuela de Ingenierías Industriales de la Universidad de

Presidente

Vocal primero
Secretario

Vocal segundo

Vocal tercero

Obteniendo la calificación de: 

Existe algo más importante que la lógica: la imaginación.

Sir Alfred Joseph Hitchcock

Director de cine

(1899-1980) 

A Noelia y Sergio 



\section{Agradecimientos}

Quiero expresar mi más sincero agradecimiento a todas las personas e instituciones que, en mayor o menor medida, han contribuido a la realización de esta Tesis Doctoral, en especial:

A mi director de Tesis, el Dr. D. Antolín Lorenzana Ibán por su orientación y apoyo en todo momento, por sus aportaciones y consejos en el conjunto de este trabajo, gracias a los cuales se ha conseguido su desarrollo, sólo superado por su calidad humana y la amistad con la que me honra.

A mi mujer, Noelia, a quien dedico la Tesis, por su inagotable cariño y motivación, por su confianza en mí y por haber estado a mi lado en todo momento.

A mis padres, hermano, suegros y a toda mi familia, por su esfuerzo y sacrificio, siempre con el único objetivo de facilitar que haga realidad mis mejores sueños.

A los profesores José Antonio Garrido García y Juan Carlos del Caño Sánchez, por brindarme la oportunidad de dedicarme a la docencia e iniciado en los caminos de la investigación cientifica.

A todos mis compañeros de Departamento, en especial a Antonio Foces, José Pereda, Pedro Olmos, Pilar Alonso, Natividad Cabreros y Belén Martínez por su disposición, compañerismo y ayuda.

Al personal del Area de Diseño Estructural de la Fundación CARTIF, en especial a Pablo López, Ali Vasallo, Carlos Lavín y Jesús de Sebastián, por el apoyo que siempre me ofrecen.

A todos ellos, muchas gracias

Mariano
Valladolid, España

Septiembre, 2010 



\section{Organización de la Tesis}

De acuerdo con la normativa vigente (Ejecución de Acuerdos de la Comisión de Doctorado de la Universidad de Valladolid de fecha 10 de mayo de 2010), esta Tesis Doctoral se presenta como compendio de publicaciones. No obstante, además de incluir los artículos publicados (Capítulo 5), también se enumeran otros trabajos realizados en el marco de esta línea de investigación, y que están en proceso de elaboración (Anexo I), para su posterior evaluación y, en su caso, publicación.

Los trabajos incluidos en este documento son:

Artículos publicados:

a) Cálculo directo de la carga crítica de pandeo de pórticos. Parte I A. Lorenzana y M. Cacho

Rev. Int. Mét. Num. Cálc. Dise. Ing., 25(3): 247-258, 2009.

b) Cálculo directo de la CARga Crítica de pandeo de pórticos. Parte II M. Cacho y A. Lorenzana

Rev. Int. Mét. Num. Cálc. Dise. Ing., 26: 31-38, 2010.

c) Optimización global de pórticos 2D CON BARRAs DE SECCión VARIABle M. Cacho Pérez y A. Lorenzana Ibán DYNA Ingeniería e Industria.

Aceptado: junio 2010.

Publicación, fecha prevista: noviembre 2010. 
Artículos en proceso de elaboración:

a) ANÁLisis LÍMITE DE PÓRTICOS PLANOS INCLUYENDO ESTABILIDAD Y COMPORTAMIENTO VIBRATORIO. PARTE I

M. Cacho y A. Lorenzana

Rev. Int. Mét. Num. Cálc. Dise. Ing.

b) AnÁlisis LÍMite DE PÓRTicos Planos INCLUYENDO ESTABILIDAD

Y COMPORTAMIENTO VIBRATORIO. PARTE II

M. Cacho y A. Lorenzana

Rev. Int. Mét. Num. Cálc. Dise. Ing.

c) Influencia de la plastificación EN la CARGa De PANDEO DE PÓRTicos 2D M. Cacho Pérez y A. Lorenzana Ibán DYNA Ingeniería e Industria.

Por último, se hace constar que el presente documento adopta el formato de Tesis Doctoral como compendio de publicaciones, y por lo tanto, consiste en una síntesis de los conceptos teóricos que sustentan los trabajos de investigación publicados o en desarrollo, en lugar del formato tradicional de documento extenso autocontenido. 


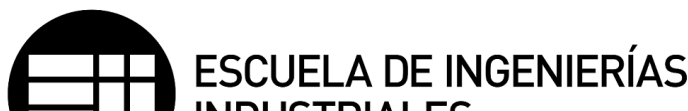 INDUSTRIALES}

El Dr. D. Antolín Lorenzana Ibán, Profesor Titular de Universidad en el Departamento de Construcciones Arquitectónicas, Ingeniería del Terreno y Mecánica de los Medios Continuos y Teoría de Estructuras de la Universidad de Valladolid:

\section{CERTIFICA}

Que D. Mariano Cacho Pérez, Ingeniero Industrial, ha realizado bajo su dirección la Tesis Doctoral titulada: "TÉCNICAS NUMÉRICAS DE ANÁLISIS LÍMITE DE PÓRTICOS PLANOS, INCLUYENDO INESTABILIDAD Y COMPORTAMIENTO VIBRATORIO" que se recoge en esta memoria para optar al grado de doctor por la Universidad de Valladolid. Así mismo, como coautor del trabajo de investigación da por escrito su conformidad a la presentación de dichos trabajos como parte de esta Tesis y por lo tanto no podrán formar parte de ninguna otra Tesis Doctoral.

Programa: Doctorado en Ingeniería Industrial (I07)

Y para que así conste, y tenga los efectos oportunos, firmo este certificado en Valladolid, a 21 de septiembre de 2010.

Dr. D. ANTOLÍN LORENZANA IBÁN 



\section{Índice de tablas}

3.1. Ejemplos. Datos comunes _. . . . . . . . . . . . . . . 38

3.2. Viga - 1.a.- Resultados - modelo 1 - caso a. . . . . . . . . . . 44

3.3. Viga - 2.a.- Resultados - modelo 2 - caso a. . . . . . . . . . . 44

3.4. Viga - 1.b.- Resultados - modelo 1 - caso b. . . . . . . . . . . 46

3.5. Viga - 2.b.- Resultados - modelo 2 - caso b. . . . . . . . . . . . 47

3.6. Viga - 1.c.- Resultados - modelo 1 - caso c. . . . . . . . . . . . . 49

3.7. Viga - 2.c.- Resultados - modelo 2 - caso c. . . . . . . . . . . . 50

3.8. Viga - 1.d.- Resultados - modelo 1 - caso d. . . . . . . . . . . . . 52

3.9. Viga - 2.d.- Resultados - modelo 2 - caso d. . . . . . . . . . . . 53

3.10. Pórtico de Lee - 1.a.- Resultados - modelo 1 - caso a. . . . . . . . . . 55

3.11. Pórtico de Lee - 2.a.- Resultados - modelo 2 - caso a. . . . . . . . . . 55

3.12. Pórtico de Lee - 1.b.- Resultados - modelo 1 - caso b. . . . . . . . . . . 55

3.13. Pórtico de Lee - 2.b.- Resultados - modelo 2 - caso b. . . . . . . . . . 55

3.14. Pórtico simple a dos aguas.- Resultados - modelo 1. . . . . . . . . 56

3.15. Pórtico simple a dos aguas.- Resultados - modelo 2 . . . . . . . . . . . 56 



\section{Índice de figuras}

2.1. Espacio de tensiones principales . . . . . . . . . . . . . . . . 15

2.2. Superficie de plastificación y superficie de rotura . . . . . . . . . . . . 16

2.3. Modelo barra 2D . . . . . . . . . . . . . . . . . . . . . . . . . . . . . . 19

2.4. Equilibrio. Configuración actual $(\mathrm{CA}) \ldots \ldots \ldots \ldots 22$

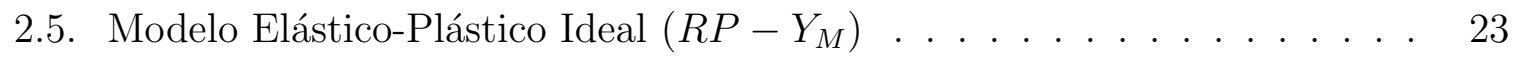

2.6. Evolución de las tensiones. . . . . . . . . . . . . . . . . . . . . . . . . 24

2.7. Función de plastificación $\left(Y_{M N}\right) \ldots \ldots \ldots \ldots$. . . . . . . . 27

2.8. Equilibrio y compatibilidad. Pórtico tipo . . . . . . . . . . . . . . . 29

3.1. Viga apoyada-empotrada. . . . . . . . . . . . . . . . . . . 38

3.2. Viga. Problema virtual/auxiliar en esfuerzos $(\mathrm{PFV})$. . . . . . . . . . . 39

3.3. Viga. Problema virtual/auxiliar en desplazamientos (PDV) . . . . . . . . 42

3.4. Viga - 1.a.- Deformada (amplificada x3600). . . . . . . . . . . . . 45

3.5. Viga - 1.a.- Diagrama de momento flector. . . . . . . . . . . . . . . . . 45

3.6. Viga - 1.a.- Modos de pandeo y vibración. . . . . . . . . . . . . . . . . 46

3.7. Viga - 1.b.- Evolución de la plastificación. . . . . . . . . . . . . . . . 47

3.8. Viga - 2.b.- Evolución de la plastificación . . . . . . . . . . . . . . . . 48

3.9. Viga - 1.b.- Evolución de la primera frecuencia propia . . . . . . . . . . . 49

3.10. Viga - 2.b.- Evolución de la primera frecuencia propia . . . . . . . . . . . 49

3.11. Viga - 1.c.- Evolución de la plastificación. . . . . . . . . . . . . . . . . . . 50

3.12. Viga - 2.c.- Evolución de la plastificación . . . . . . . . . . . . . . . 51

3.13. Viga - 1.c.- Evolución de la primera frecuencia propia . . . . . . . . . . 51

3.14. Viga - 2.c.- Evolución de la primera frecuencia propia . . . . . . . . . . . 52

3.15. Pórtico de Lee . . . . . . . . . . . . . . . . . . . . . 54

3.16. Pórtico simple a dos aguas . . . . . . . . . . . . . . 57 



\section{Resumen}

En este trabajo se presentan técnicas matemáticas y numéricas de análisis no lineal de pórticos atendiendo a distintos criterios que condicionan su utilización desde el punto de vista práctico, a medida que aumenta la carga hasta el colapso. En principio, dichas técnicas se aplican a pórticos metálicos planos constituidos por barras rectas esbeltas. Además de los métodos de análisis estático, para determinar esfuerzos y desplazamientos, se incluye, de forma tentativa, la capacidad de evaluar las cargas de pandeo y las frecuencias de vibración, así como los modos asociados. Con todo, se pretende hacer un seguimiento de una gran cantidad de magnitudes para cada estado de carga, hasta el colapso, y así tener caracterizada la estructura tanto estática como dinámicamente. Algunas de estas magnitudes son fácilmente observables o medibles mediante técnicas experimentales (OMA, EMA). De esta manera se abre la posibilidad de aplicar el trabajo a la evaluación de la vulnerabilidad y a técnicas de monitorizado y salud estructural incluyendo localización y evaluación de daño.

Por estos motivos, se ha considerado importante perseguir este objetivo tentativo y no se ha encontrado en la literatura trabajos similares con los que comparar, ni a nivel numérico ni experimental. Sólo mediante estudios, desarrollos o experimentos posteriores podremos conocer lo acertado de las teorías, métodos y procedimientos numéricos empleados en este trabajo. 



\begin{abstract}
This research presents numerical and mathematical techniques for nonlinear analysis of frames according to different criteria restricting their applicability, from the practical point of view, as the load increases until the collapse. These techniques are applied to 2D steel frames of straight slender beams. Besides the static analysis techniques to determine stress and deflections the ability to evaluate the buckling loads, natural frequencies and corresponding vibration modes is tentatively included. The objective is to know the response (static and dynamic one) of the structure beyond linear elastic behavior . Some of analyzed factors are easily observable or measurable by experimental methods, mainly the related ones with dynamical properties.

With these work we try to make a tentative proposal to monitoring techniques, structural health monitoring and damage assessment and damage location. No similar approaches have been found in the literature. Hence, only through further research we will able to know the accuracy of the proposed techniques.
\end{abstract}





\section{Índice general}

1. Introducción 1

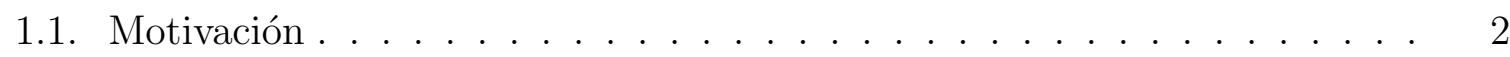

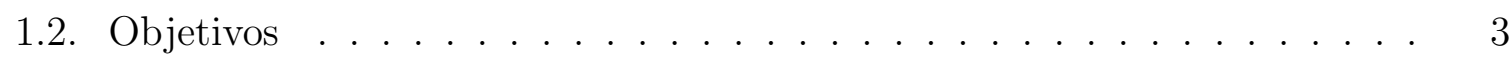

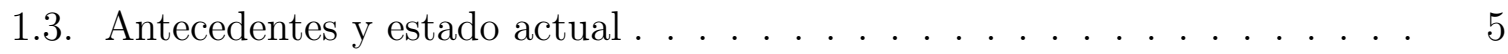

2. Metodología 13

2.1. Plasticidad . . . . . . . . . . . . . . . . . . . . . 14

2.1.1. Criterio de plastificación . . . . . . . . . . . . . . . 14

2.1.2. Ley de flujo . . . . . . . . . . . . . . . . . 17

2.2. Modelo barra 2D . . . . . . . . . . . . . . . . . . . 18

2.2.1. Equilibrio. Configuración Inicial $(\mathrm{CI}) \ldots \ldots \ldots \ldots$

2.2.2. Equilibrio. Configuración Actual $(\mathrm{CA}) \ldots \ldots \ldots \ldots$

2.2.3. Comportamiento Elástico-Lineal . . . . . . . . . . . . . . . . 21

2.2.4. Comportamiento Elástico-Plástico Ideal $\left(R P-Y_{M}\right) \ldots \ldots \ldots .22$

2.2.5. Comportamiento Elastoplástico $\left(S A-Y_{M N}\right) \ldots \ldots \ldots \ldots$

2.3. Análisis de pórticos . . . . . . . . . . . . . . . . . . . . . . 29

2.3.1. Equilibrio y compatibilidad . . . . . . . . . . . . . . 29

2.3.2. Condiciones de contorno . . . . . . . . . . . . . . 30

2.3.3. Análisis límite . . . . . . . . . . . . . . . . . . . . . . . 31

2.3.4. Estabilidad . . . . . . . . . . . . . . . . . . 32

2.3.5. Comportamiento vibratorio $\ldots \ldots \ldots \ldots$

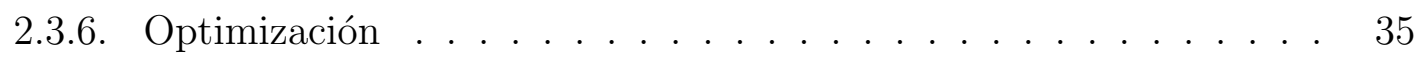

3. Resultados y Discusión $\quad 37$

3.1. Ejemplo 1: Viga apoyada-empotrada . . . . . . . . . . . 38 
3.1.1. Métodos clásicos . . . . . . . . . . . . . . . . 39

3.1.2. Método propuesto . . . . . . . . . . . . . . 43

3.2. Ejemplo 2: Pórtico de Lee . . . . . . . . . . . . . . . . . 54

3.3. Ejemplo 3: Pórtico simple a dos aguas . . . . . . . . . . . . 56

4. Conclusiones $\quad 59$

$\begin{array}{ll}\text { 5. Artículos publicados } & 65\end{array}$

$\begin{array}{lr}\text { Bibliografía } & 107\end{array}$

$\begin{array}{ll}\text { Anexo } & 110\end{array}$

$\begin{array}{ll}\text { I. Trabajos en desarrollo } & 111\end{array}$ 


1

\section{Introducción}

1.1. Motivación ........................ 2

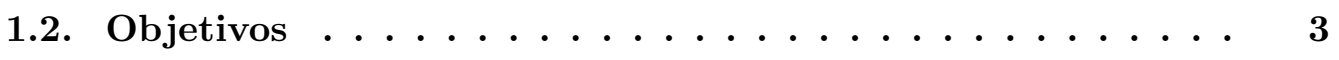

1.3. Antecedentes y estado actual $\ldots \ldots \ldots \ldots$ 
En este primer capítulo se indican las distintas motivaciones dentro de la amplia línea de investigación del comportamiento de las estructuras que justifican el presente trabajo, los objetivos concretos, y por último, los antecedentes más relevantes y el estado actual.

\subsection{Motivación}

A continuación se indican los alcances técnicos de los bloques de conocimiento más representativos del trabajo:

1. Inestabilidad/Pandeo con deformaciones de flexión en el plano de la estructura. Para ello se plantea el equilibrio del conjunto en su configuración deformada/actual en hipótesis de pequeños desplazamientos (teoría de segundo orden) y se determina la menor carga crítica y el modo de pandeo asociado. Se ha planteado el problema con generalidad suficiente para poder considerar, entre otros, los siguientes casos: cargas puntuales y momentos concentrados, cualquier tipo de carga distribuida, peso propio y carga térmica e incluso barras no prismáticas, es decir, de propiedades estáticas (canto, área, inercia, etc.) variables a lo largo de la longitud de la barra. Así mismo, dado un pórtico plano, definido por geometría, material, propiedades estáticas, cargas, apoyos y libertades, se ha estudiado el problema de optimización que consiste en buscar qué variación de la geometría de los perfiles maximiza la carga crítica en base a un número discreto de parámetros.

2. Comportamiento vibratorio. Es muy importante para el ingeniero de estructuras disponer de la información teórica de las primeras frecuencias de vibración y sus correspondientes modos para poder determinar la respuesta vibratoria del conjunto. Ya que la mayoría de las técnicas experimentales que se usan para caracterizar el estado en cada momento de las estructuras existentes se basan en el uso de acelerómetros para registrar vibraciones ambientales (técnicas OMA) o vibraciones inducidas por excitadores (técnicas EMA). De la correlación entre la información teórica y la experimental se podrá determinar la situación de la estructura aplicando técnicas de identificación modal, por ejemplo. Conocida dicha situación, se podrá determinar la capacidad remanente, o la conveniencia de ejecutar refuerzos o intervenciones en estructuras dañadas. 
3. Análisis límite/Cálculo plástico/No linealidad material. Todo lo anterior puede ser aplicado a estructuras donde para un determinado estado de cargas pueda haberse superado el régimen elástico lineal. Se considera, en base a criterios recogidos en las normativas (Código Técnico de Edificación, Eurocódigos, etc.) el análisis plástico en base al modelo tensión-deformación elástico-perfectamente plástico. Además se acepta que la plastificación es localizada/concentrada en base al momento plástico reducido, por el efecto del esfuerzo axil, dada por la función de plastificación $Y_{M N}$. Atendiendo a consideraciones de compatibilidad y equilibrio, esto da lugar al acoplamiento de los grados de libertad plásticos longitudinal y rotacional en cada sección plastificada. Por todo ello se emplea el término "sección agotada por plastificación" en lugar del término clásico de "rótula plástica" como fue puesto de manifiesto en los trabajos previos del equipo de investigación ${ }^{8,50}$.

\subsection{Objetivos}

Hoy en día para el conocimiento y cuantificación del comportamiento límite de estructuras de barras es imprescindible disponer de modelos numéricos que permitan una adecuada modelización de los complejos fenómenos no lineales que tienen lugar incluso en régimen estático. El rápido desarrollo informático de los últimos años ha hecho posible que se pueda simular el comportamiento estructural límite con bastante precisión. Estado límite es la situación en la que la estructura o parte de ella deja de comportarse de forma adecuada bajo condiciones normales (estados límite de servicio) o colapsa bajo cargas accidentales severas (estados límite últimos).

Es bien conocido que la forma más eficiente de realizar una modelización numérica del comportamiento lineal de las estructuras de barras es mediante elementos monodimensionales. Y el uso de este tipo de elementos para problemas en los que existe no linealidad del material está muy limitado, fundamentalmente porque las formulaciones utilizadas asumen hipótesis muy simplificadas. Ante esta situación, es deseable desarrollar un elemento simple pero riguroso, que permita abordar eficientemente el estudio del comportamiento elastoplástico en estructuras de barras hasta los estados límite de colapso plástico e inestabilidad.

El trabajo plantea modelos basados en el comportamiento plástico a nivel de punto, pero expresado en función de las variables tradicionales del modelo 1D de barra de 
Navier-Bernoulli. Se llega, tras ciertas hipótesis, al concepto de sección agotada, en lugar del de rótula plástica, cumpliendo el modelo creado con todas las propiedades teóricas del comportamiento plástico. A este modelo se ha incorporando la no linealidad geométrica que permita tener en cuenta el análisis de la estabilidad. De esta manera se podría conocer o evaluar la vulnerabilidad y la seguridad de las estructuras frente a acciones accidentales, siendo éste un tema de alto interés en ingeniería estructural. En este marco, los objetivos concretos se pueden resumir en los siguientes puntos:

1. Se persigue el desarrollo de métodos numéricos que permitan alcanzar objetivos de gran interés práctico como evaluar la seguridad desde el punto de vista resistente de un determinado diseño estructural ante cualquier carga (incluyendo acciones accidentales).

2. Desarrollo de nuevas formulaciones para tener en cuenta los estados límite de inestabilidad y extender el modelo monodimensional de sección agotada a casos prácticos en ingeniería de estructuras.

3. Aplicación de los resultados al diseño, dimensionamiento y optimización de estructuras de barras. Conocer cómo evoluciona la estructura a medida que la carga progresa. Así pues, es importante conocer en qué partes de la estructura se puede permitir la plastificación y cuáles han de ser reforzadas.

En la determinación de estos objetivos hemos tratado de ser ambiciosos y de plantear el método de resolución con la mayor rigurosidad posible. Somos conscientes de la complejidad de la formulación del problema de inestabilidad junto con el comportamiento elastoplástico, tanto a nivel teórico como en todo lo relativo a su implementación numérica. Más cuando no hemos encontrado en la literatura trabajos con objetivos similares. En este documento presentamos un primer acercamiento al establecimiento de un método incremental para este problema no lineal. 


\subsection{Antecedentes y estado actual}

Es conocida la importancia que tienen los sistemas estructurales de barras en muchos campos de la ingeniería y su capacidad de soportar más carga de aquella para la que fueron diseñados. Esto en parte se debe a los coeficientes de seguridad exigidos por las normativas (NBE, CTE, ECs, LRFD, etc.) y también a que su diseño suele estar basado en el comportamiento elástico y para satisfacer estados límites de servicio ${ }^{40,54}$, en vez de estar basado en régimen plástico y para estados límites últimos. La determinación de esta reserva de resistencia permitiría, por ejemplo, realizar un diseño más optimizado de la estructura, conocer el factor de seguridad real ante ciertas sobrecargas, realizar una evaluación de la vulnerabilidad de estructuras existentes ante determinados estados límites últimos, o bien proponer sistemas eficientes de control de estructuras.

Es imprescindible disponer de modelos numéricos que permitan una adecuada simulación de los complejos fenómenos no lineales que tienen lugar incluso en régimen estático, a partir de los cuales se pueda conocer y cuantificar el comportamiento límite de estructuras de barras. Desde el punto de vista computacional la forma más eficiente de realizar un modelado numérico del comportamiento lineal de las estructuras de barras es mediante elementos finitos monodimensionales tipo barra (BEAM) ${ }^{13,31,39}$. Este elemento finito reproduce fielmente el comportamiento de las barras descrito en textos clásicos de Resistencia de Materiales ${ }^{36,61}$, bien sea el modelo de barra de Navier-Bernoulli o el de Timoshenko.

Lógicamente, mediante el Método de los Elementos Finitos ${ }^{22,25,31}$ también se puede abordar el estudio del comportamiento plástico de las estructuras de barras. Desde el punto de vista computacional existen múltiples modelos matemáticos que buscan la descripción numérica del fenómeno de adaptación plástica de las estructuras hasta su colapso. Existen actualmente tres formas de realizar dicho estudio:

a) Mediante el uso de elementos finitos no monodimensionales. En este caso, la barra se modela mediante elementos tipo placa (SHELL), o tipo sólido (SOLID). Incluso pueden encontrarse estudios en los que la barra se modela con elementos de elasticidad plana (PLANE2D) en tensión o deformación plana ${ }^{61}$.

b) Mediante el uso de elementos tipo barra, aunque no estrictamente monodimensionales. Existen formulaciones ${ }^{11,13}$ de barras elastoplásticas en las que para describir su 
comportamiento no es suficiente con el conocimiento de los desplazamientos y esfuerzos de la línea media de la sección de la barra $(\mathrm{s}, 0,0)$, sino que también es necesario conocer magnitudes en los puntos $(\mathrm{s}, \mathrm{y}, \mathrm{z})$ no pertenecientes a la directriz de la misma.

c) Mediante elementos monodimensionales tipo barra, pero con simplificaciones ${ }^{34}$ que como se sabe no pueden reproducir fielmente el comportamiento plástico real. Las bases clásicas de este planteamiento se recogen en distintos textos ${ }^{23}$ y existen multitud de publicaciones ${ }^{6,33,58}$ desde los años 80 que utilizan este modelo, incluido o sugerido también en varias normativas de edificación.

Mediante los planteamientos a) y b) se puede llegar, al menos en teoría, a describir el comportamiento límite de una estructura de barras hasta el colapso. Sin embargo, el esfuerzo computacional es muy alto y usualmente aparecen problemas de convergencia a partir de la plastificación completa de la primera sección.

Los modelos monodimensionales no lineales hasta ahora utilizados son extremadamente sencillos y no satisfacen ciertas condiciones básicas del comportamiento plástico de los materiales, pudiéndose llegar a soluciones que no están en equilibrio, y a secciones en las que se supone que existe rótula plástica en las que el momento flector no tiene el valor del momento plástico reducido ${ }^{11,17,26}$. Es deseable pues contar con una formulación simple pero rigurosa para abordar eficientemente este tipo de comportamiento no lineal.

De forma resumida y desde el punto de vista teórico, el comportamiento elastoplástico de un determinado elemento finito viene usualmente gobernado por teorías de primer orden a través de la matriz de rigidez elastoplástica tangente ${ }^{18,22,39,42,57}$, e intervienen la matriz de rigidez elástica y las derivadas de la función de plastificación con respecto a las tensiones. La extensión de esta formulación al caso de barras lleva a una expresión similar en la que juega un papel fundamental la función de plastificación y sus derivadas con respecto a los esfuerzos ${ }^{20,34,38}$. Esta función expresa la combinación de esfuerzos que llevan a la plastificación completa de la sección. Para casos simples en los que se considere que esta función depende sólo del momento flector, la formulación lleva estrictamente al modelo tradicional de rótula plástica. Sin embargo, si de manera adicional se considera la influencia de los esfuerzos axil y/o cortante, los desarrollos son más complejos y aparecen fenómenos de acoplamiento entre esfuerzos y desplazamientos ${ }^{50}$. Es de destacar que, a pesar de que la formulación de matriz elastoplástica para barras es conocida desde hace tiempo, el estudio e interpretación de los resultados con ella obtenidos no han sido 
realizados por ningún grupo de investigación, pues no se han encontrado en la literatura referencias al acoplamiento entre esfuerzos y entre desplazamientos a que da lugar.

Para estructuras de edificación, las normativas en general (nacionales o extranjeras, vigentes o futuras) incluyen varias restricciones y exigen ciertas comprobaciones. El diseño bajo estas restricciones es sencillo, ya que generalmente es suficiente realizar un análisis elástico. Pero si las cargas son más grandes o las resistencias más pequeñas, las estructuras pueden empezar a perder sus propiedades resistentes, aparecer ciertos daños y quedar eventualmente inservibles. Hay muchas razones por las que pueden aparecer cargas superiores a las que se utilizaron para el diseño elástico (ciertas sobrecargas de uso, cargas accidentales, cambio de finalidad de la estructura, sismos, etc.) y razones por las que la resistencia puede disminuir (envejecimiento, fatiga, fuego ${ }^{59}$, etc.). La evaluación bajo estas condiciones no es tan fácil como en el caso de análisis elástico. Los métodos de análisis límite de estructuras ${ }^{60}$ aun no están lo suficientemente desarrollados y concretamente, como ya se ha dicho, interesa el desarrollo de un método avanzado y riguroso con este fin, aplicable por el momento a estructuras planas de barras en régimen estático.

Las estructuras construidas con un material de unas características adecuadas de ductilidad pueden seguir soportando cargas crecientes pese a que en algún lugar el material haya abandonado el régimen elástico. Si el proceso de carga continúa, las secciones son sometidas a tensiones cada vez mayores hasta que finalmente se llega al colapso o agotamiento resistente, siendo ésta otra causa de fallo estructural y la que nos ocupa en este trabajo.

El comportamiento plástico del material está definido por una deformación que se caracteriza por ser en parte irreversible, independiente del tiempo y dependiente de la historia de la carga. De esta forma, si se aumenta de manera progresiva el estado de cargas, después de un comportamiento elástico inicial en el que las tensiones y deformaciones son proporcionales, aparece un comportamiento plástico del material en el que se rompe esa proporción. Este comportamiento sólo se manifiesta cuando se ha conseguido un cierto nivel de tensión, nivel que varía con el estado de deformación inicial del material, la temperatura y el proceso de carga, de acuerdo con los resultados experimentales. La fluencia plástica del material de la estructura produce pérdidas de rigidez que afectan de manera considerable a las tensiones y deformaciones. Todo ello ocasiona una menor capacidad resistente del material y la menor capacidad portante de la sección y de la 
estructura en su conjunto.

El proceso de determinación de la carga de colapso o carga última es conocido actualmente como análisis límite o cálculo plástico, por contraposición al análisis elástico, basado en la consideración de que ningún punto material de la estructura abandone el régimen elástico. En su favor decir que permite obtener diseños más racionales, una notable economía de los materiales, etc. La evaluación de la carga última de la estructura es esencial para asegurar el cumplimiento de las condiciones que garanticen la funcionalidad, estabilidad y durabilidad de la misma.

En definitiva, dado un medio continuo con unas determinadas condiciones de sustentación y sometido a un determinado conjunto de cargas, el objetivo del análisis límite consiste en encontrar el menor valor de la carga que provocaría el colapso, o lo que es lo mismo, la mínima carga para la que los desplazamientos no serían admisibles.

Pues bien, la Teoría de la Elasticidad fue desarrollada por autores como Bernoulli (1667-1748), Hooke (1635-1703), Euler (1707-1783), Coulomb (1736-1806), Navier (17851836) y Cauchy (1789-1857) entre otros, que obtuvieron las ecuaciones fundamentales y desarrollaron el concepto de tensor de tensiones y deformaciones. Sin embargo, la teoría del comportamiento plástico del material no comenzó a desarrollarse hasta 1864 cuando Tresca (1814-1885) publicó una serie de experimentos sobre extrusión en los que planteaba la fluencia plástica del metal, si bien para el caso del estudio de suelos ya había sido indicada por autores como Coulomb, Poncelet (1788-1867) y Rankine (1820-1872).

No hubo desarrollos significativos hasta finales del siglo XIX en que Von Mises (18831953) y Hencky (1855-1952) desarrollaron nuevos criterios para la plastificación de materiales basados en que la fluencia se produce cuando la energía de deformación, asociada a la componente desviadora del tensor de tensiones, alcanza un valor crítico. En los años 30, Bridgman experimentó sobre flujos a altas presiones, obteniendo relaciones entre el cambio de volumen porcentual y la presión aplicada.

Cuando cada punto de un sólido está sometido a incrementos proporcionales de tensiones las leyes de comportamiento pueden ser integradas, obteniéndose relaciones entre las tensiones totales y deformaciones plásticas totales. Mientras que las leyes de comportamiento integrales dan lugar al método paso a paso de análisis, las leyes totales permiten en ciertos casos prescindir de la historia de carga y dan lugar a métodos directos de análisis, en 1924, Hencky introdujo tales leyes. Kacinczy, en 1914, fue el primero en investigar la reserva de resistencia plástica existente en una estructura hiperestática, 
introduciendo los conceptos de rótula plástica y mecanismo de colapso.

Los primeros autores que trataron el análisis límite fueron Van den Brock, Barker, Horne, Heyman, Neal, Beedle, Heyman y Hodge; entre 1955 y 1960. Este tema fue impulsado posteriormente por Greenberg, Prager y Hill, que establecieron de forma rigurosa los teoremas básicos.

A partir de esta época, y debido a la aparición del ordenador, hubo un relanzamiento de los estudios en dos direcciones: la aplicación de la teoría a la resolución de problemas utilizando métodos numéricos y el desarrollo de nuevas teorías para problemas y materiales complejos. El primer trabajo que aplicó programación lineal al análisis límite de estructuras fue debido a Charnes y Greenberg en 1951; a partir del cual dicha herramienta matemática se aplicó sistemáticamente a los distintos problemas de cálculo plástico, análisis límite directo, cargas variables, análisis límite incremental, grandes desplazamientos y diseño. Como autores contemporáneos dentro de este campo se destacan los textos de Alarcón ${ }^{1}$, Doblaré ${ }^{13}$, Cañas ${ }^{6}$ y Neal ${ }^{34}$, que desarrollan estudios estructurales teniendo en cuenta las condiciones últimas o de colapso de las estructuras.

En base a los postulados iniciales del comportamiento plástico del material y utilizando los métodos numéricos asociados a los procesos informáticos (siendo el que más popularidad ha alcanzado el Método de los Elementos Finitos, MEF), distintos autores desarrollan procesos de cálculo para aplicarlos a las estructuras de barras. En este campo, en la época actual, destacan las aportaciones de autores como Tin-Loi ${ }^{2,29,54,55}$, Wong ${ }^{60}$ y Möller ${ }^{31}$, entre otros.

Convencionalmente, para los casos en los que se considere plastificación en función sólo del momento flector, esta formulación lleva estrictamente al modelo tradicional de rótula plástica (Neal y Symonds ${ }^{46}$; Takeda et al. ${ }^{48}$; Maier et al. ${ }^{28}$; Cohn y Franchi ${ }^{10}$; Roufaiel y Meyer $\left.{ }^{41}\right)$.

Por otro lado, el comportamiento mecánico de elementos comprimidos ha sido una fuente de aportaciones teóricas y de resultados prácticos de gran interés para la ingeniería del que han surgido importantes áreas como la estabilidad estructural. El primer científico que se ocupó de estudiar el comportamiento resistente de un elemento prismático fue Galileo en su publicación de 1638, donde trata de obtener algunos resultados sobre la resistencia de una viga sometida a cargas transversales, este problema, y, en particular, el de determinación de la elástica o deformada de la viga, es conocido como el problema de Galileo. Respecto al problema de pandeo, J. Bernoulli (1705) obtiene de manera precisa 
la ecuación de la elástica basándose en la hipótesis de Mariotte sobre la fibra neutra. Euler, en 1744, mediante su método de cálculo de variaciones obtiene, a partir de la sugerencia de D. Bernoulli, la ecuación diferencial de la elástica y obtiene también el valor de la carga de pandeo. En 1757, obtiene de nuevo el valor de dicha carga a partir de una simplificación (ecuación linealizada) de la ecuación diferencial de la elástica. En publicaciones posteriores, considera el caso de piezas de sección variable y otros con carga axial distribuida a lo largo de la longitud de la pieza. Al mismo tiempo se profundiza

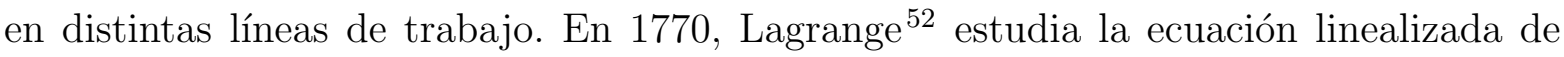
Euler e investiga el valor de las cargas de pandeo superiores a la primera para la pieza biarticulada. Así mismo, se propuso hallar la forma que debería tener una columna de altura y volumen dados para que aguantase la máxima compresión posible sin pandear, y establece erróneamente que la mejor forma es un cilindro circular, en otras palabras, que el éntasis no aumenta la resistencia. Posteriormente, Clausen, en 1851, determina la pieza óptima frente al pandeo, la forma de columna más estable es aquélla donde la variación de la sección circular a lo largo del fuste lo constituye una curva parecida a una cicloide $^{56}$. En relación con este problema, hay multitud de publicaciones de gran interés. Keller $^{21}$ y Tadjbakhsh ${ }^{47}$, derivaron que las formas geométricas óptimas de la pieza más resistente frente al fenómeno de pandeo es una cuya sección varía de la misma forma que la determinada por Clausen, pero, en lugar de secciones circulares, éstas son triángulos equiláteros que mantienen el paralelismo de los lados y con el baricentro en el eje de la pieza.

Taylor ${ }^{49}$ estudió el mismo problema usando un enfoque energético, y presentó un límite inferior para el máximo autovalor. Spillers y Levy ${ }^{45}$ extendieron el problema del pandeo de una columna al del diseño óptimo de una placa frente a inestabilidades por flexión y más tarde al estudio del fenómeno de pérdida de estabilidad de una cubierta cilíndrica simétrica a lo largo de un eje ${ }^{44}$. Sin embargo, un inconveniente con todos estos trabajos es que sus respectivos autores limitaron sus diseños óptimos a sólo una restricción: un volumen constante. En la práctica, sin embargo, las restricciones impuestas por la resistencia del material empleado o por los desplazamientos juegan un papel igualmente importante.

Fu y Ren ${ }^{15}$ retomaron los trabajos antes mencionados, aunque agregando las restricciones de tensiones necesarias, planteando así el problema de minimizar el volumen de una columna sujeta a una cierta carga mediante el ajuste de su forma geométrica. El 
método que utilizaron para resolver este problema fue el gradiente reducido generalizado, obteniendo resultados muy favorables. Existen también referencias muy recientes, que emplean la teoría matemática del análisis funcional para obtener la forma óptima frente a pandeo por torsión y/o flexión a partir de las condiciones que establece el principio de Pontriyagin, Atanackovic ${ }^{3}$.

Como se ha podido comprobar, el campo de la optimización estructural ha atraído la atención de un gran número de investigadores desde hace tiempo. Sin embargo, en relación al estudio de fenómenos de inestabilidad, los trabajos en general se limitan al análisis de barras aisladas y consideran muy pocas restricciones de diseño, la de volumen y en el mejor de los casos se añade la comprobación a resistencia.

Los estudios realizados al estudiar el problema de inestabilidad, abordado desde un planteamiento matemáticamente preciso, basado inicialmente en la integración directa de las ecuaciones diferenciales que describen el problema que deriva del planteamiento del equilibrio en configuración deformada lleva a distintas técnicas numéricas ${ }^{5,24}$.

Por último, relativo al comportamiento vibratorio del conjunto del sistema estructural, recordar como es bien sabido, que queda caracterizado por sus frecuencias naturales o propias y los correspondientes modos de vibración asociados, tal y como se puede consultar en bibliografía clásica de vibraciones mecánicas ${ }^{4,14,19,30,51}$. 

2

\section{Metodología}

2.1. Plasticidad ...................... 14

2.1.1. Criterio de plastificación . . . . . . . . . . . . . . . . 14

2.1.2. Ley de flujo . . . . . . . . . . . . . . . 17

2.2. Modelo barra 2D . . . . . . . . . . . . . . 18

2.2.1. Equilibrio. Configuración Inicial (CI) . . . . . . . . . . . 18

2.2.2. Equilibrio. Configuración Actual (CA) . . . . . . . . . . . . 21

2.2.3. Comportamiento Elástico-Lineal . . . . . . . . . . . 21

2.2.4. Comportamiento Elástico-Plástico Ideal $\left(R P-Y_{M}\right) \ldots \ldots 22$

2.2.5. Comportamiento Elastoplástico $\left(S A-Y_{M N}\right) \ldots \ldots$

2.3. Análisis de pórticos . . . . . . . . . . . . . 29

2.3.1. Equilibrio y compatibilidad . . . . . . . . . . . . . 29

2.3.2. Condiciones de contorno . . . . . . . . . . . . . . 30

2.3.3. Análisis límite . . . . . . . . . . . . . . . . . . . 31

2.3.4. Estabilidad . . . . . . . . . . . . . . . . . 32

2.3.5. Comportamiento vibratorio . . . . . . . . . . . 33

2.3.6. Optimización . . . . . . . . . . . . . . . 35 


\subsection{Plasticidad}

\subsubsection{Criterio de plastificación}

La información experimental, ensayo de tracción y los resultados de los ensayos de Lode entre otros, evidencia que la hipótesis de comportamiento lineal elástico del material sólo es razonable para un determinado rango de valores de las cargas. En este apartado se resume el criterio que predice el comienzo de la plastificación en un estado general de tensión tridimensional. La tensión es una magnitud que se introduce en el modelo matemático de la Teoría de la Elasticidad para explicar cómo se transmiten a través de los sólidos las fuerzas aplicadas.

Cada porción de sólido estará en equilibrio, admitido el postulado de distribución continua de fuerzas, de unidades fuerza por unidad de superficie, constituye el concepto de tensión, y es la hipótesis fundamental de la Mecánica de los Medios Continuos. El vector tensión se puede descomponer en las direcciones normal y tangente al plano, obteniéndose las componentes intrínsecas normal $(\sigma)$ y tangencial $(\tau)$ de la tensión, respectivamente. Cada una de sus componentes se pueden descomponer respecto de un sistema de referencia ortonormal de ejes $(s, y, z)$. La definición de tensión en un punto requiere conocer el plano donde actúa, por lo que para su determinación se utiliza una magnitud tensorial de segundo orden denominada tensor de tensiones de Cauchy o tensiones verdaderas $\left(\sigma_{i j}\right)$.

Para cada una de las medidas de la deformación se obtiene una definición distinta del concepto de tensión. En la Teoría Lineal de la Elasticidad, asociado al modelo de deformación ingenieril, se define la tensión sin distinguir la configuración de estudio ya que siempre se toma como tal la configuración indeformada, sin embargo en el análisis no lineal debe precisarse en qué configuración se define la tensión. Si se desea obtener las fuerzas interiores en la configuración deformada en términos del área indeformada o inicial, se requiere una nueva magnitud denominada tensor de primeras tensiones de Piola-Kirchhoff $\left(T_{i j}\right)$. Así mismo, la magnitud que permite obtener las componentes de las fuerzas interiores en configuración indeformada por unidad de superficie de dicha configuración se denomina tensor de segundas tensiones de Piola-Kirchhoff $\left(S_{i j}\right)$.

Como el tensor de tensiones $\left(\sigma_{i j}\right)$ es simétrico, los autovalores son números reales y los autovectores ortogonales entre sí. El conjunto de autovalores se denomina tensio- 
nes principales $\left(\sigma_{1}, \sigma_{2}, \sigma_{3}\right)$, y los autovectores direcciones principales $\left(e_{i}\right)$. La ecuación característica se puede desarrollar en función de los invariantes del sistema, lineal $\left(I_{1}\right)$, asociado a la variación de volumen del entorno de un punto, cuadrático $\left(I_{2}\right)$ y cúbico $\left(I_{3}\right)^{12}$.

Dadas las componentes $\left(\sigma_{i j}\right)$ del tensor de tensiones en un punto, es conveniente considerar su descomposición en suma de dos nuevos tensores, a los que llamaremos medio (o esférico) y desviador (véase la figura 2.1). La división del estado de tensiones principales en las componentes hidrostática y desviadora viene justificada en que el cambio de volumen en materiales metálicos está determinado exclusivamente por el tensor esférico, asociado a la tensión normal octaédrica media, que no influye en el proceso de plastificación, mientras que la magnitud que gobierna la plastificación viene dada sólo por el tensor desviador, asociado a la tensión tangencial octaédrica media.

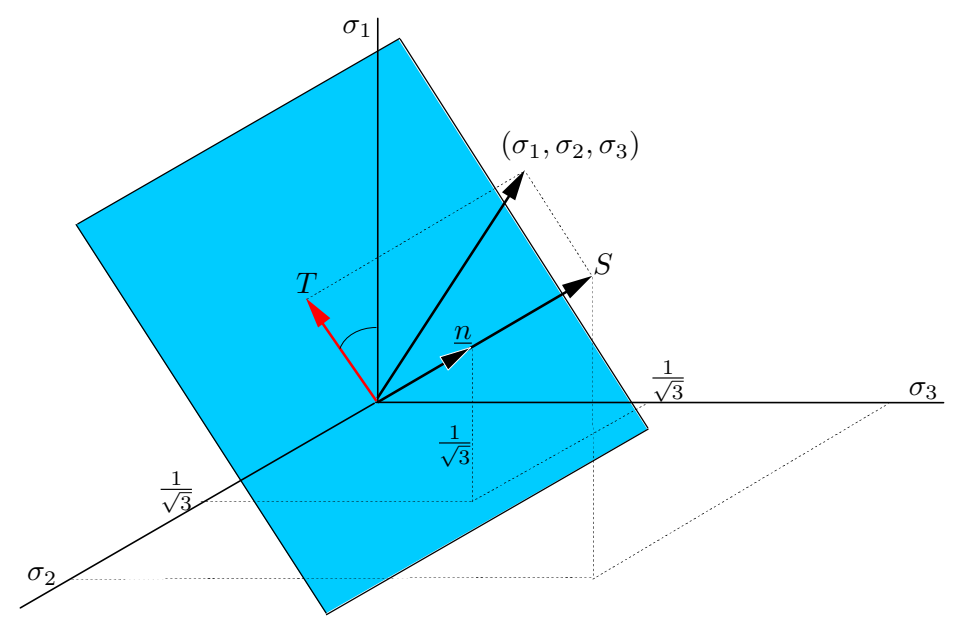

Figura 2.1: Espacio de tensiones principales

En resumen, se espera que la plastificación se produzca cuando cierta función del estado de tensión y de la deformación plástica acumulada alcance cierto valor, que dependerá de las propiedades del material y de la temperatura. Por tanto, la forma general de un criterio de plastificación será:

$$
F\left(\sigma_{i j}, \varepsilon_{i j}^{p}\right) \leq C\left(\sigma_{F}(\kappa), T\right)
$$


siendo $(\kappa)$ el grado de rigidización del material.

La superficie de plastificación es la superficie dada por una ecuación del tipo (2.1) en el espacio de tensiones principales, sin ordenar dichas tensiones principales de mayor a menor, lo que conduce a simetrías (así por ejemplo, para el acero, material isótropo con idéntico comportamiento a tracción que a compresión implica repetitividad cada $60^{\underline{O}}$ ). Admitiendo que la superficie de plastificación depende sólo de la parte desviadora del tensor de tensiones, la superficie de plastificación será un prisma recto cuyo eje es la diagonal principal de los ejes coordenados, y la forma de la sección del prisma será la que proponga cada criterio de plastificación particular. Superficie que además debe ser convexa según los requisitos de comportamiento del material en régimen plástico, figura 2.2 .

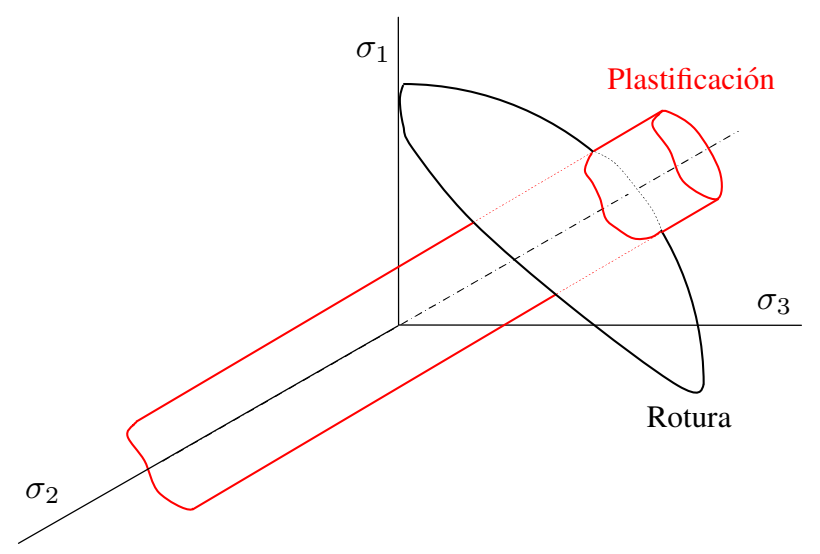

Figura 2.2: Superficie de plastificación y superficie de rotura

En realidad, el comportamiento similar en tracción y en compresión habitualmente observado en los metales, estará limitado en la zona de tracciones por la superficie de rotura, lugar geométrico de los puntos del espacio de tensiones principales en que se produce la rotura del material. Esta superficie es independiente de la superficie de plastificación, ya que está asociada a un fenómeno distinto.

Los resultados experimentales indican que la plastificación dependerá del valor de la densidad de energía de deformación debida al tensor desviador, que se denomina energía de distorsión. El criterio de Von Mises propone que la plastificación ocurrirá cuando dicho término de energía alcance cierto valor límite, para fijar ese valor se toma como referencia el ensayo de tracción. Y así obtenemos la expresión del criterio de Von Mises 
en ejes principales (2.2) y en función de tensiones no principales (2.3):

$$
\begin{gathered}
\left(\sigma_{1}-\sigma_{2}\right)^{2}+\left(\sigma_{1}-\sigma_{3}\right)^{2}+\left(\sigma_{2}-\sigma_{3}\right)^{2} \leq 2 \sigma_{F}^{2} \\
\left(\sigma_{11}-\sigma_{22}\right)^{2}+\left(\sigma_{11}-\sigma_{33}\right)^{2}+\left(\sigma_{22}-\sigma_{33}\right)^{2}+6\left(\sigma_{12}^{2}+\sigma_{13}^{2}+\sigma_{23}^{2}\right) \leq 2 \sigma_{F}^{2}
\end{gathered}
$$

Puede apreciarse que la representación del criterio anterior en el espacio de tensiones principales es un cilindro de sección circular cuyo eje forma ángulos iguales con los tres ejes coordenados. Comparado con los resultados de Lode el criterio de Von-Mises se ajusta satisfactoriamente a los resultados experimentales y es ampliamente utilizado para materiales metálicos.

\subsubsection{Ley de flujo}

El apartado anterior establece el comienzo de la plastificación, mientras que la ley de flujo define la variación de las deformaciones durante el comportamiento plástico, relaciona la magnitud y dirección de las deformaciones plásticas con la de los incrementos de las tensiones.

$\mathrm{Al}$ producirse un incremento infinitesimal en las tensiones y superado el estado límite elástico aparecen incrementos infinitesimales de deformaciones $d \varepsilon_{i j}^{e p}$ denominadas deformaciones elastoplásticas, de las cuales una parte corresponde a las deformaciones elásticas $d \varepsilon_{i j}^{e}$, y otra a las deformaciones plásticas $d \varepsilon_{i j}^{p}$. Considerando el principio de la descomposición aditiva se tiene:

$$
d \varepsilon_{i j}^{e p}=d \varepsilon_{i j}^{e}+d \varepsilon_{i j}^{p}
$$

La determinación de la ley de flujo se basa en las hipótesis elementales propuestas por Drucker en 1951, estos postulados parten de los conceptos de endurecimiento por deformación y material estable. El endurecimiento por deformación o acritud es un fenómeno que aparece en puntos materiales en los que la tensión ha superado el límite elástico, produciendo el aumento de dicho límite y generando variaciones en la superficie de plastificación. A los materiales que presentan este tipo de comportamiento se les denomina estables y en ellos el trabajo de las fuerzas durante el proceso de carga es siempre positivo (lo que implica la propiedad de convexidad para la superficie de plastificación).

Von Mises fue el primero en sugerir que los incrementos de deformación plástica están relacionados con la superficie de plastificación, siendo la hipótesis más aceptada la del 
principio de ortogonalidad o normalidad ${ }^{7,26}$ :

$$
d \varepsilon_{i j}^{p}=d \lambda \frac{d F}{d \sigma_{i j}}
$$

Para materiales estables el factor $(d \lambda)$ es un escalar positivo denominado multiplicador plástico que para el caso de flujo asociado tiene la expresión:

$$
d \lambda=\frac{1}{h} \frac{\left(\frac{\partial F}{\partial \sigma_{i j}} d \sigma_{i j}\right)}{\frac{\partial F}{\partial \sigma_{i j}} \frac{\partial F}{\partial \sigma_{i j}}}
$$

donde el parámetro $(h)$ relaciona el incremento de tensión con el incremento de deformación plástica, siendo proporcional al módulo plástico del ensayo de tracción.

\subsection{Modelo barra 2D}

Las limitaciones impuestas al modelo son: aplicación a estructuras planas cargadas sobre el plano, barras de sección bisimétrica, plasticidad ideal (sin endurecimiento), obtención de las tensiones equivalentes mediante la hipótesis de Von Mises y modelo de barra de Navier-Bernoulli. Así mismo, se adopta un estado proporcional de cargas, de tal manera que todas las acciones sobre la estructura, excepto el peso propio y la carga térmica, se incrementan en igual proporción respecto a sus valores nominales, mediante el factor de carga $(\lambda)$. En base a estas hipótesis se presenta a continuación la ecuaciones de equilibrio, compatibilidad y comportamiento para el elemento barra tipo bajo estudio.

\subsubsection{Equilibrio. Configuración Inicial (CI)}

Para el análisis del pórtico plano, se dibuja el diagrama de sólido libre de cada barra y se plantea el equilibrio de fuerzas y momentos. Para ello resulta necesario definir un sistema de referencia cartesiano para cada barra, sistema local de la barra denotado por $(s, y, z)$. De la condición de equilibrio en el plano resulta el siguiente sistema de ecuaciones diferenciales lineales en términos de los esfuerzos verdaderos o esfuerzos de 
Cauchy (véase la figura 2.3):

$$
\left\{\begin{array}{l}
N_{s}^{\prime}(s)+q_{s}(s)=0 \\
V_{y}^{\prime}(s)+q_{y}(s)=0 \\
M_{z}^{\prime}(s)+V_{y}(s)=0
\end{array}\right.
$$

donde las variables con notación prima indican derivada respecto de la coordenada espacial $(s)$.

Otro concepto importante es el de grado de libertad, se denominan grados de libertad a las variables independientes que definen completamente la configuración deformada del sistema. En los sistemas formados por barras esbeltas es habitual tomar los desplazamientos y giros de los nudos como grados de libertad del problema.

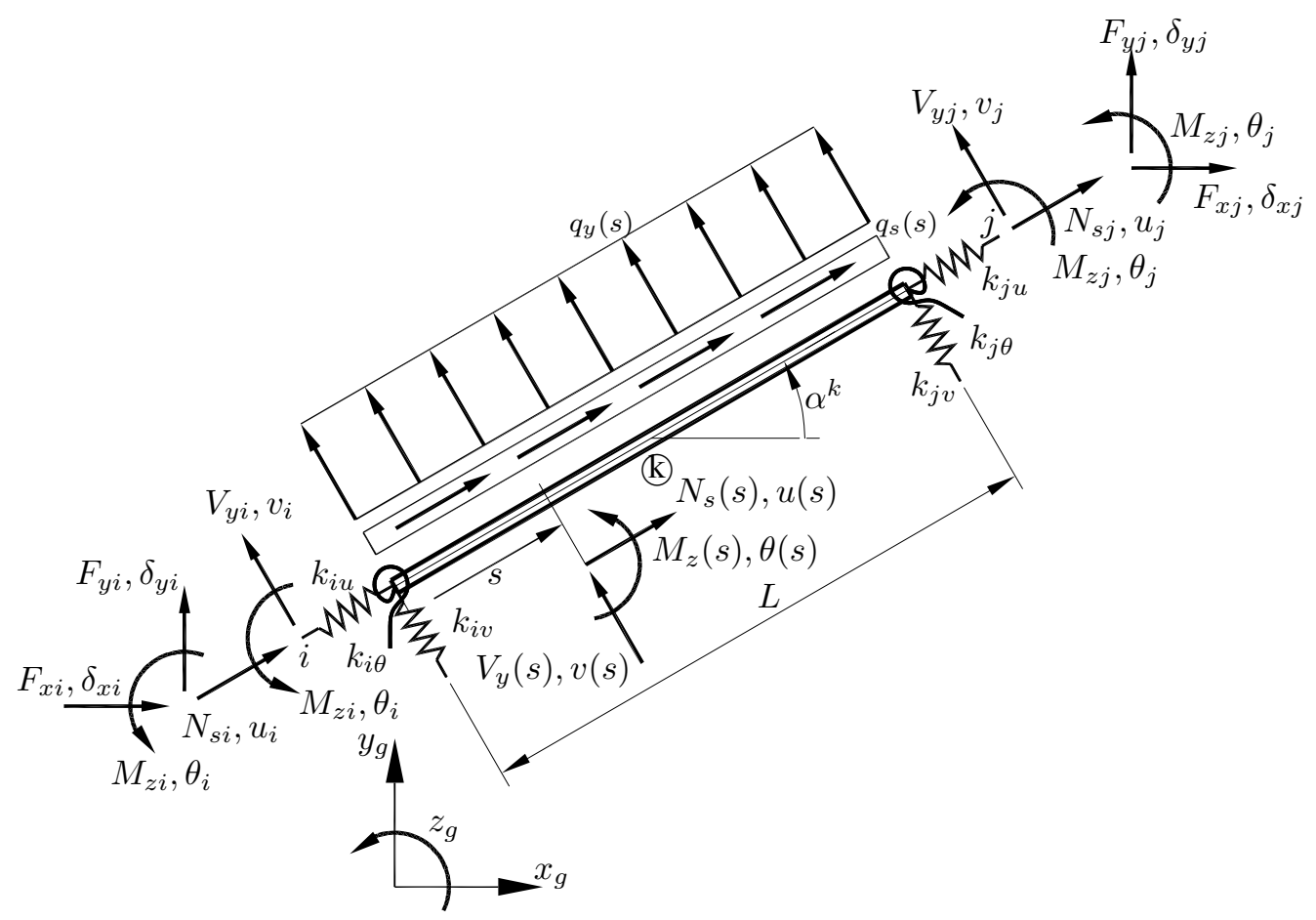

Figura 2.3: Modelo barra 2D

Y así, se definen los esfuerzos y los grados de libertad, en coordenadas locales en los 
extremos de la barra como:

$$
\begin{gathered}
\left\{\begin{array}{l}
\underline{E}_{i}^{k}=\left(N_{s i}, V_{y i}, M_{z i}\right)^{T}=\left(N_{s}(0), V_{y}(0), M_{z}(0)\right)^{T} \\
\underline{E}_{j}^{k}=\left(N_{s j}, V_{y j}, M_{z j}\right)^{T}=\left(N_{s}(L), V_{y}(L), M_{z}(L)\right)^{T}
\end{array}\right. \\
\left\{\begin{array}{l}
\underline{u}_{i}^{k}=\left(u_{i}, v_{i}, \theta_{i}\right)^{T}=(u(0), v(0), \theta(0))^{T} \\
\underline{u}_{j}^{k}=\left(u_{j}, v_{j}, \theta_{j}\right)^{T}=(u(L), v(L), \theta(L))^{T}
\end{array}\right.
\end{gathered}
$$

donde $(L)$ es la longitud de la barra $(k)$.

Se definen las rigideces en el extremo de cada barra en el sistema de coordenadas local:

$$
\underline{\underline{k}}_{l}^{k}=\left[\begin{array}{ccc}
k_{l u}^{k} & 0 & 0 \\
0 & k_{l v}^{k} & 0 \\
0 & 0 & k_{l \theta}^{k}
\end{array}\right]=\left[\begin{array}{ccc}
f_{l u}^{k} & 0 & 0 \\
0 & f_{l v}^{k} & 0 \\
0 & 0 & f_{l \theta}^{k}
\end{array}\right] \cdot\left[\begin{array}{ccc}
\frac{E A}{L} & 0 & 0 \\
0 & \frac{12 E I_{z}}{L^{3}} & 0 \\
0 & 0 & \frac{4 E I_{z}}{L}
\end{array}\right] ; \quad l=i, j
$$

Por otro lado, la matriz de cambio de base que permite pasar las magnitudes del problema del sistema global de la estructura $\left(x_{g}, y_{g}, z_{g}\right)$ al sistema local de cada barra $(s, y, z)$, se define por:

$$
\underline{\underline{L}}^{k}=\left[\begin{array}{ccc}
\cos \alpha^{k} & \operatorname{sen} \alpha^{k} & 0 \\
-\operatorname{sen} \alpha^{k} & \cos \alpha^{k} & 0 \\
0 & 0 & 1
\end{array}\right]
$$

Con lo cual, las fuerzas y momentos, y los desplazamientos y giros, en el sistema global $\left(x_{g}, y_{g}, z_{g}\right)$ vienen dados por las siguientes expresiones:

$$
\begin{gathered}
\underline{Q}_{l}^{k}=\left(F_{x l}, F_{y l}, M_{z l}\right)^{T}=\left(\underline{\underline{L}}^{k}\right)^{T} \underline{E}_{l}^{k} ; \quad l=i, j \\
\underline{\delta}_{l}^{k}=\left(\underline{\underline{L}}^{k}\right)^{T}\left(\underline{u}_{l}^{k}+\left(\underline{\underline{k}}_{l}^{k}\right)^{-1}\left(\underline{E}_{l}^{k}-\underline{F}_{l}^{k}\right)\right) ; \quad l=i, j
\end{gathered}
$$

donde $\left(\underline{F}_{l}^{k}\right)$ es la solicitación de tipo concentrado/puntual en el nudo $(l)$ de la barra $(k)$.

El modelo de barra 2D empleado considera en sus secciones extremas (zonas de unión de los elementos barra) nudos semirrígidos de rigidez longitudinal, transversal y rotacional dada. Se ha optado por este tipo de elemento en lugar del clásico de nudos rígidos por dos motivos fundamentales: el primero, porque permite incluir de forma muy 
sencilla cualquier tipo de libertad elástica (así, por ejemplo, libertad de desplazamiento relativo longitudinal en el nudo de la izquierda del elemento, se asigna al factor $f_{1 u}^{i}$ el valor $10^{-9}$, cero numérico) , y en segundo lugar, porque la variación del valor de la rigidez rotacional en un nudo de la discretización durante el proceso de carga permite introducir de forma simple una rótula plástica o una sección agotada en la estructura (así, por ejemplo, la formación de rótula plástica a la derecha del elemento se simula reduciendo la rigidez a un valor numérico relativo muy pequeño, al igual que antes, al valor cero numérico, $f_{2 \theta}^{j}=10^{-9}$ ).

\subsubsection{Equilibrio. Configuración Actual (CA)}

Debido a que un análisis de estabilidad requiere que el equilibrio se plantee en la configuración real, es decir, en la deformada, se hace necesario fijar el sistema de referencia al que referir desplazamientos y esfuerzos de la barra. Para ello, se considera un sistema de referencia cartesiano de orientación fija para cada barra independiente de su deformación, sistema global de la barra que denotamos $(X, Y, Z)$. Del equilibrio de fuerzas según los ejes $(X, Y)$ y de momentos según $(Z)$, resulta el siguiente sistema de ecuaciones diferenciales en términos de los pseudo esfuerzos o esfuerzos de Piola-Kirchhoff ${ }^{32}$ (véase la figura 2.4):

$$
\left\{\begin{array}{l}
H^{\prime}(s)+q_{X}(s)=0 \\
V^{\prime}(s)+q_{Y}(s)=0 \\
M^{\prime}(s)-H(s) \theta(s)+V(s)=0
\end{array}\right.
$$

donde las variables con notación prima indican derivada respecto de la coordenada espacial $(s)$, y donde se ha aproximado el seno del ángulo por el ángulo y su coseno por la unidad en base a la hipótesis de pequeños desplazamientos.

\subsubsection{Comportamiento Elástico-Lineal}

Se asume la hipótesis de pequeños desplazamientos y pequeñas deformaciones, y se considera que el comportamiento del material es de tipo elástico y lineal. Las ecuaciones que relacionan los esfuerzos $\left(N_{s}, V_{y}, M_{z}\right)$ con los desplazamientos $(u, v, \theta)$ de un punto de 


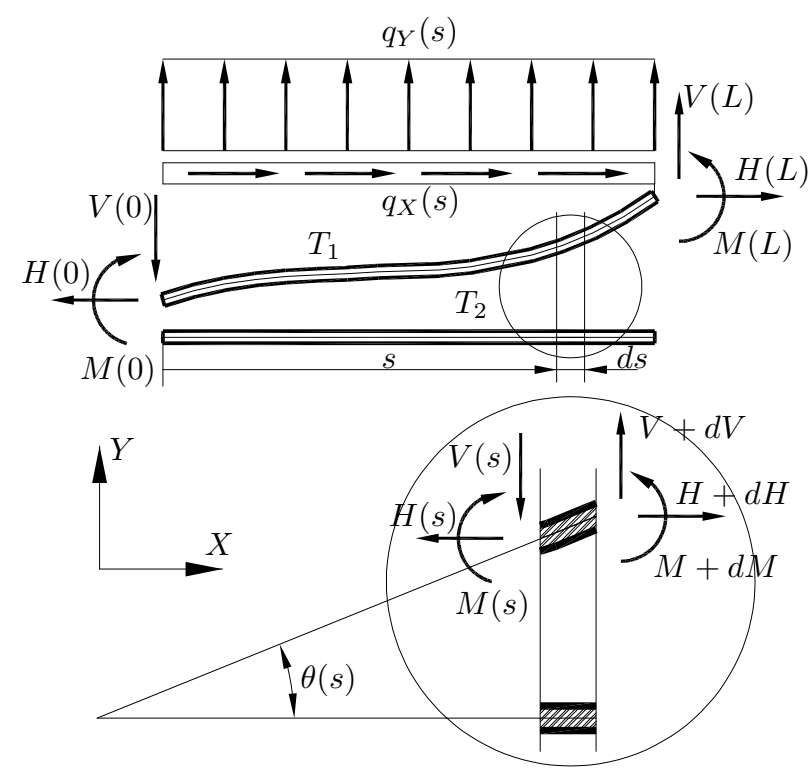

Figura 2.4: Equilibrio. Configuración actual (CA)

la directriz de la barra son:

$$
\left\{\begin{array}{l}
N_{s}(s)=E A(s)\left(u^{\prime}(s)-\frac{\alpha}{2}\left(T_{1}+T_{2}\right)\right) \\
\theta(s)=v^{\prime}(s) \\
M_{z}(s)=E I_{z}(s)\left(\theta^{\prime}(s)+\frac{\alpha}{h(s)}\left(T_{1}-T_{2}\right)\right)
\end{array}\right.
$$

siendo $E$ el módulo de Young del material, $I_{z}(s)$ y $A(s)$ el momento de inercia y el área de cada sección transversal de la barra. Se incluye el efecto térmico, suponiendo por simplicidad variación lineal de la temperatura en el canto $h(s)$, entre $T_{1}$ y $T_{2}$, siendo $\alpha$ el coeficiente de dilatación térmica.

\subsubsection{Comportamiento Elástico-Plástico Ideal $\left(R P-Y_{M}\right)$}

El proceso de plastificación se va a desarrollar en el dominio de la sección de la barra por lo que habrá que determinar la forma de aplicar la Teoría de la Plasticidad en el entorno del punto al dominio de la sección.

A continuación, se realizará el estudio clásico de rótula plástica, la equivalencia estática de las tensiones que aparecen sobre las fibras de la sección en comportamiento elas- 
toplástico determina los esfuerzos de la sección en la barra recta, axil, cortante y flector:

$$
\left\{\begin{array}{l}
N_{s}(s)=\int_{A} \sigma_{s}(s, y) d A \\
V_{y}(s)=\int_{A} \tau_{s y}(s, y) d A \\
M_{z}(s)=\int_{A} \sigma_{s}(s, y) y d A
\end{array}\right.
$$

Se considera que comienza la plastificación en algún punto de una barra cuando la tensión equivalente $\left(\sigma_{\text {eqv }}\right)$ obtenida según alguno de los criterios de plastificación, alcanza el valor de la tensión de fluencia $\left(\sigma_{F}\right)$, véase la figura 2.5.

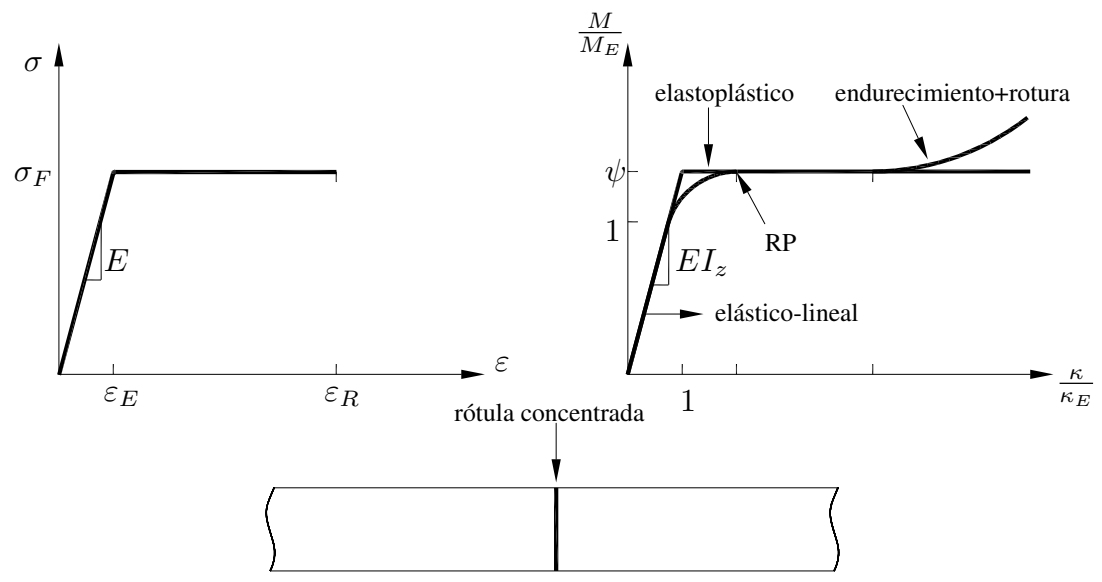

Figura 2.5: Modelo Elástico-Plástico Ideal $\left(R P-Y_{M}\right)$

Al alcanzar la plastificación se modifica el comportamiento del punto material ya plastificado, afectando éste a los que se encuentren en su entorno, de forma que según vaya aumentando la solicitación sobre el sistema se irá extendiendo el dominio plástico en la sección. Si se siguen aumentando las cargas, la plasticidad sigue penetrando en la sección afectando cada vez a más fibras, hasta que todas las fibras de la sección alcanzan la tensión límite o máxima, caso que aparece en secciones afectadas únicamente por tensiones normales y se considera que la sección ha plastificado de forma completa. En la determinación de la plastificación completa de una sección se tendrá en cuenta la hipótesis de plastificación concentrada súbita, por lo que la plasticidad sólo afecta a la sección que alcanza la plastificación completa y no a las de su entorno, además, al aparecer de forma súbita no se considera el comportamiento elastoplástico transitorio en 
el dominio de la sección. El estudio clásico que tiene en cuenta únicamente el efecto del momento flector implica la formación de una rótula plástica. Si no se tiene en cuenta el efecto del endurecimiento en la plastificación esta rótula plástica produce libertad de giro en la sección, lo cual modifica el comportamiento de todo el sistema, tanto desde el punto de vista de los desplazamientos como del reparto de esfuerzos (figuras 2.5 y 2.6).

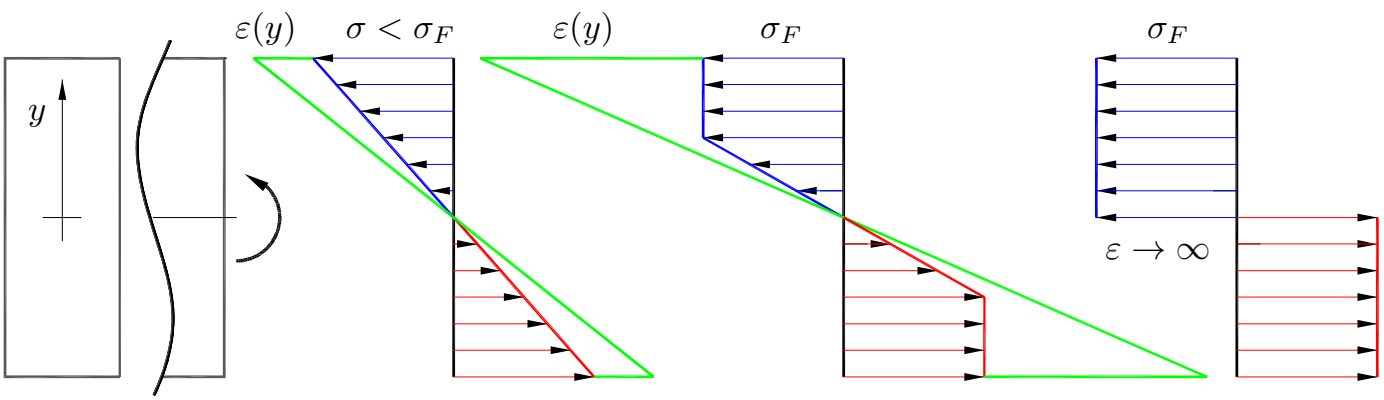

Figura 2.6: Evolución de las tensiones.

Considerando la teoría de Navier-Bernoulli se obtiene la expresión de las tensiones normales y tangenciales en régimen elástico que aparecen en barras sometidas a solicitaciones de tracción-flexión. Para el estudio respecto de un sistema de referencia asociado al centro de gravedad de la sección en ejes principales de inercia, la expresión, bien conocida viene dada por:

$$
\left\{\begin{array}{l}
\sigma_{s}(s, y)=\frac{N_{s}(s)}{A}+\frac{M_{z}(s)}{I_{z}} y \\
\tau_{s y}(s, y)=\frac{V_{y}(s) Q_{y}(y)}{b(y) I_{z}}
\end{array}\right.
$$

donde $Q_{y}(y)$ es el momento estático respecto del eje $(z)$ de la porción de la sección hasta la fibra en estudio y $b(y)$ es el ancho de la sección en dicha cota $(y)$.

A partir de estas fórmulas se comprueba que el reparto de tensiones normales y tangenciales es función de la geometría de la sección por lo que los esfuerzos necesarios para la aparición de una rótula plástica dependen de dicha geometría, y por lo tanto es importante determinar la relación entre los esfuerzos y el avance de la plastificación en la sección, la variación de posición de la línea neutra y el estado de plastificación completa.

El criterio de plastificación utilizado va a ser el de Von Mises-Hencky (2.2)/(2.3), en el que la tensión equivalente $\left(\sigma_{\text {eqv }}\right)$ para un estado de tensión dado en coordenadas 
cartesianas viene expresado en componentes por:

$$
\sigma_{e q v}=\sqrt{\sigma_{s}^{2}+3 \tau_{s y}^{2}}
$$

Ahora bien, al aumentar el estado de carga aumenta el momento flector $\left(M_{z}(s)\right)$ y por lo tanto la tensión normal $\left(\sigma_{s}(s, y)\right)$ de manera que el límite elástico se alcanza cuando esta tensión coincida con la de fluencia $\left(\sigma_{F}\right)$. Las fibras en las que comienza la plastificación son las más alejadas de la línea neutra por lo que en el comienzo de la plastificación se tiene:

$$
M_{E}=\sigma_{F} \frac{2 I_{z}}{h}=W \sigma_{F}
$$

expresión en la que $\left(M_{E}\right)$ es el momento elástico, que corresponde al momento máximo que puede soportar la sección sin que se produzca plastificación en ningún punto de la misma, $(h)$ el canto de la sección y $(W)$ la característica geométrica de la sección denominada módulo resistente.

Si el momento aumenta por encima del momento elástico $\left(M_{E}\right)$ las tensiones y deformaciones variarán, generándose un comportamiento elastoplástico del material de la sección, según el cual las deformaciones siguen aumentando pero las tensiones no sobrepasan el valor de la tensión de fluencia $\left(\sigma_{F}\right)$, por lo que en las fibras donde la tensión haya alcanzado este valor se produce la plastificación, y existen dos dominios de comportamiento: elástico y plástico, perfectamente diferenciados.

Si se sigue aumentando el momento flector el comportamiento plástico se propaga hacia la línea neutra hasta llegar a la situación en la que ninguna fibra de material trabaja ya en régimen elástico, correspondiente a la plastificación completa de la sección, instante en el que se considera formada la rótula plástica (véase la figura 2.6). Al momento flector que produce la plastificación total de la sección se le denomina momento plástico $\left(M_{P}\right)$, de expresión:

$$
M_{P}=\int_{A} \sigma_{F} y d A=\sigma_{F} \int_{A} y d A=S \sigma_{F}
$$

donde $(S)$ es el denominado módulo plástico y corresponde al doble del momento estático de media sección respecto de la línea neutra (para el caso de sección con al menos una simetría).

Admitiendo la hipótesis de deformación de Navier por la que las secciones planas normales a la directriz de la pieza $(s)$ antes de la deformación permanecen planas y 
normales a dicha directriz después de la deformación. Para la situación de plastificación completa de la sección, la penetración de las fibras plastificadas llega hasta la línea neutra, por lo que el radio de curvatura se anula y las deformaciones unitarias y la curvatura teóricamente alcanzan el valor infinito, lo que nos indica que el momento plástico nunca se puede llegar a alcanzar. Aún así, el criterio según el cual en una sección sometida a un estado de flexión aparece una rótula plástica cuando el momento aplicado a la sección coincide con el momento plástico sigue siendo un criterio actual y aceptable (véase la figura 2.5). Aunque en general, en el instante en que se ha generado la rótula plástica, como consecuencia de la actuación simultánea de los dos esfuerzos, flector y axil, la magnitud del momento máximo con la que se alcanza la plasticidad completa de la sección será menor que el momento plástico, y se denomina momento plástico reducido $\left(M_{P N}\right)$.

\subsubsection{Comportamiento Elastoplástico $\left(S A-Y_{M N}\right)$}

Anteriormente se ha introducido el caso de no linealidad del material con comportamiento elastoplástico en el entorno de un punto para el caso en que no exista endurecimiento y que el planteamiento esté asociado a la superficie de plastificación. Se va a particularizar ahora para el dominio de barras, que da lugar al concepto de sección agotada por plastificación o sección plastificada que considera composición de los esfuerzos axil y momento flector de forma acoplada.

El proceso de plastificación comienza cuando la fibra sometida a mayor tensión alcance el valor de fluencia $\left(\sigma_{F}\right)$. La posición de la línea neutra varía según la plasticidad penetra en la sección, variación que se puede obtener planteando la condición de equilibrio de esfuerzos en la dirección longitudinal de la barra.

Como consecuencia de la actuación simultánea de los dos esfuerzos, flector y axil, la capacidad de la sección para transmitir esfuerzo flector será menor que el momento plástico $\left(M_{P}\right)$. La relación entre el momento flector y el esfuerzo axil necesarios para la plastificación completa de una sección con geometría determinada se denomina función de plastificación $\left(Y_{M N}\right)$.

Para el caso de una barra de sección rectangular constante de canto $(h)$ y ancho $(b)$, si se consideran de forma independiente los esfuerzos flector y axil de plastificación $\left(M_{P}\right)$ 
y $\left(N_{P}\right)$ respectivamente, se tiene:

$$
M_{P}=\frac{b h^{2}}{4} \sigma_{F} ; \quad N_{P}=b h \sigma_{F}
$$

Para el caso de comportamiento elastoplástico del material y fase plástica con combinación de esfuerzos se obtiene la función de plastificación $\left(Y_{M N}\right)$ de la sección rectangular teniendo en cuenta los efectos de los esfuerzos flector y axil:

$$
Y_{M N}=\frac{M_{z}}{M_{P}}+\left(\frac{N_{s}}{N_{P}}\right)^{2}-1=0
$$

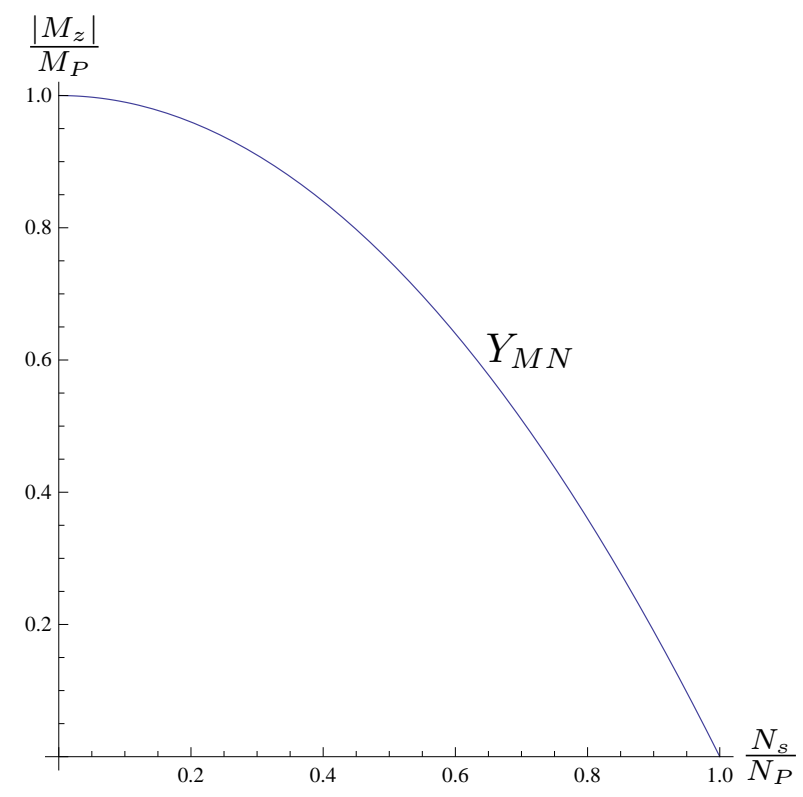

Figura 2.7: Función de plastificación $\left(Y_{M N}\right)$

Respecto a la particularización de la ley de flujo (2.5) se parte de que en el periodo de comportamiento elastoplástico para el dominio tipo barra, la variación de desplazamientos en los extremos se puede descomponer en una componente elástica y una componente plástica $^{7,26,50}$, tal y como ya se indico anteriormente, que se expresa de forma vectorial:

$$
\begin{gathered}
d \underline{u}^{e p}=d \underline{u}^{e}+d \underline{u}^{p} \\
d \underline{u}^{p}=\left(d u_{p}(0) 0 d \theta_{p}(0) d u_{p}(L) 0 d \theta_{p}(L)\right)^{T}
\end{gathered}
$$


La variación del desplazamiento plástico $\left(d \underline{u}^{p}\right)$, teniendo en cuenta las leyes para el caso de flujo asociado se puede expresar:

$$
d \underline{u}^{p}=\frac{1}{h} \underline{n}\left(\underline{n}^{T} \cdot d \underline{E}\right)
$$

siendo $(d \underline{E})$ el vector de esfuerzos en los extremos de la barra, de componentes:

$$
d \underline{E}=\left(d N_{s}(0) 0 d M_{z}(0) d N_{s}(L) 0 d M_{z}(L)\right)^{T}
$$

Si se considera el vector normal $(\underline{n})$ a la superficie de plastificación $\left(Y_{M N}\right)$ en función del gradiente de la función de plastificación

$$
\underline{n}=\frac{\left(\frac{\partial Y_{M N}}{\partial \underline{E}}\right)}{\sqrt{\left(\frac{\partial Y_{M N}}{\partial \underline{E}}\right) \cdot\left(\frac{\partial Y_{M N}}{\partial \underline{E}}\right)}}
$$

Se obtiene así, la respuesta elastoplástica en términos de la función de plastificación $\left(Y_{M N}\right)$ y de los esfuerzos de la sección $(\underline{E})$. El gradiente de la función de plastificación, cuyos términos aparecen al derivar la función de plastificación aplicada a los nodos plastificados respecto de los esfuerzos de los nodos de la barra resulta, para el caso de sección rectangular:

$$
\left(\frac{\partial Y_{M N}}{\partial \underline{E}}\right)=\left[\begin{array}{cccccc}
\frac{2 N_{s}(0)}{N_{P}} & 0 & 1 & 0 & 0 & 0 \\
0 & 0 & 0 & \frac{2 N_{s}(L)}{N_{P}} & 0 & 1
\end{array}\right]^{T}
$$

Si se impone la condición de que en una sección agotada ante carga adicional su estado de esfuerzos debe moverse por la curva de plastificación se llega a las siguientes expresiones de la ley de plastificación en el dominio de la barra para el caso de plastificación sin endurecimiento (parámetro $h=0$ ):

$$
\left\{\begin{array}{l}
d M_{z}(i)=-\left(2 N_{s}(i)-d N_{s}(i)\right) \frac{M_{z}(i)}{\left|M_{z}(i)\right|} \frac{M_{P}}{N_{P}^{2}} d N_{s}(i) \\
d u_{p}(i)=\frac{2 N_{s}(i)}{N_{P}} \frac{M_{z}(i)}{\left|M_{z}(i)\right|} d \theta_{p}(i)
\end{array} \quad i=0, L\right.
$$

y resulta sencillo la incorporación de este tipo de expresiones al modelo de barra (figura 2.3) como condiciones de contorno en sus extremos. 


\subsection{Análisis de pórticos}

En la sección anterior, se ha indicado el conjunto de ecuaciones que describe el comportamiento de la barra aislada, y en este apartado, se describe el método de análisis que permite determinar la respuesta del sistema de barras que forma el pórtico plano. Para ello, se necesita una referencia común junto con las condiciones de equilibrio y compatibilidad en los nudos de unión de las distintas barras.

\subsubsection{Equilibrio y compatibilidad}

Siempre que se analizan estructuras compuestas por más de una barra, es necesario establecer un sistema de referencia común a todas las barras (sistema de referencia/ejes global de la estructura $\left.\left(x_{g}, y_{g}, z_{g}\right)\right)$. Para expresar las magnitudes monodimensionales de cada barra en el sistema de referencia común a toda la estructura, se realiza el correspondiente cambio de base en función de la orientación inicial de las barras, dado por el ángulo $\alpha_{b}$, ángulo que forma el eje local de cada barra con el eje $\left(x_{g}\right)$ del sistema global.

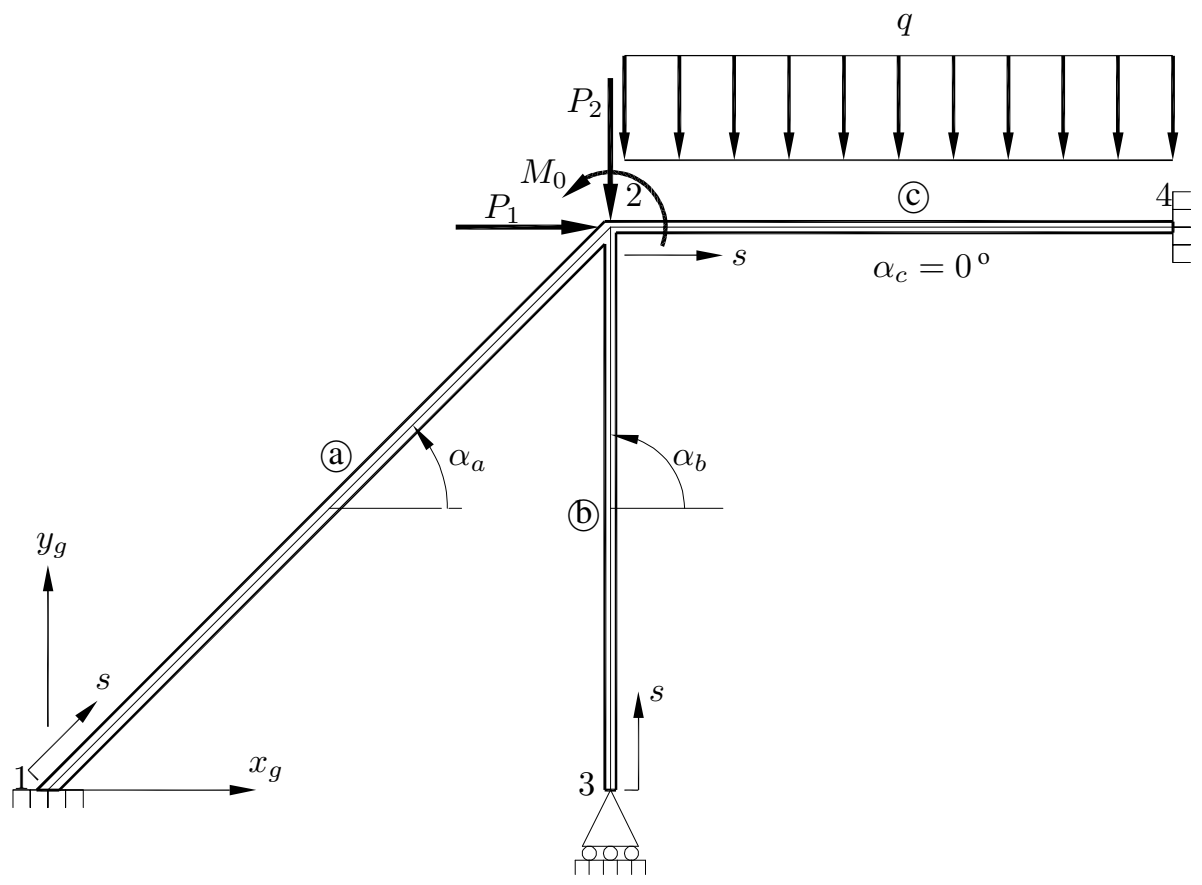

Figura 2.8: Equilibrio y compatibilidad. Pórtico tipo

Seguidamente, es necesario discretizar la estructura/pórtico, es decir, dividir la estruc- 
tura en un número adecuado de barras. A diferencia de los métodos clásicos de análisis, basados en un planteamiento matricial, en este trabajo se ha optado por describir cada barra por su correspondiente ecuación diferencial, lo que se ha denominado método implícito de análisis estructural, se trata de un método de análisis directo al no requerir mallados ni matriz de rigidez alguna de manera que se consigue una mayor generalidad. Debido a la formulación empleada y en base a las hipótesis asumidas es suficiente con emplear un número de barras mínimo, un único elemento tipo barra 2D por cada tramo recto entre nudos.

Tras estas operaciones, sólo resta imponer en cada uno de los nudos de la estructura las correspondientes condiciones de compatibilidad de desplazamientos y giros, y de equilibrio de fuerzas y momentos. Este proceso, aunque sencillo, es de casuística muy variada dependiendo de las libertades consideradas en cada nudo. En la aplicación informática desarrollada se ha conseguido sistematizar para que una vez definidos los datos del problema (geometría, perfiles, materiales, cargas, apoyos, libertades, etc.) se realice de forma automática sin la intervención del usuario gracias a la formulación del elemento barra 2D de nudos semirrígidos. Así por ejemplo, para el pórtico tipo de la figura 2.8, resulta:

$$
\left\{\begin{array} { l } 
{ \underline { F } _ { 1 } = \underline { Q } _ { 1 } ^ { a } = ( R _ { x 1 } , R _ { y 1 } , M _ { z 1 } ) ^ { T } } \\
{ \underline { F } _ { 2 } = \underline { Q } _ { 2 } ^ { a } + \underline { Q } _ { 2 } ^ { b } + \underline { Q } _ { 2 } ^ { c } = ( P _ { 1 } , - P _ { 2 } , M _ { 0 } ) ^ { T } } \\
{ \underline { F } _ { 3 } = \underline { Q } _ { 3 } ^ { b } = ( 0 , R _ { y 3 } , 0 ) ^ { T } } \\
{ \underline { F } _ { 4 } = \underline { Q } _ { 4 } ^ { c } = ( R _ { x 4 } , R _ { y 4 } , M _ { z 4 } ) ^ { T } }
\end{array} \quad \left\{\begin{array}{l}
\underline{\delta}_{1}=\underline{\delta}_{1}^{a}=(0,0,0)^{T} \\
\underline{\delta}_{2}=\underline{\delta}_{2}^{a}=\underline{\delta}_{2}^{b}=\underline{\delta}_{2}^{c}=\left(\delta_{x 2}, \delta_{y 2}, \theta_{2}\right)^{T} \\
\underline{\delta}_{3}=\underline{\delta}_{3}^{b}=\left(\delta_{x 3}, 0, \theta_{3}\right)^{T} \\
\underline{\delta}_{4}=\underline{\delta}_{4}^{c}=(0,0,0)^{T}
\end{array}\right.\right.
$$

donde $\left(\delta_{x 2}, \delta_{y 2}, \delta_{x 3}, \theta_{2}, \theta_{3}\right)$ son los grados de libertad del pórtico, $\left(R_{x 1}, R_{y 1}, M_{z 1}, R_{y 3}, R_{x 4}, R_{y 4}, M_{z 4}\right)$ las reacciones, es decir, las incógnitas estáticas, y $\left(P_{1}, P_{2}, M_{0}\right)$ las cargas aplicadas.

\subsubsection{Condiciones de contorno}

A la vista del orden del sistema de ecuaciones diferenciales $(2.7) /(2.14)$ y $(2.15)$, es necesario imponer seis condiciones de contorno por barra en desplazamientos y/o esfuerzos. Como es sabido del teorema de unicidad $^{12,53}$, en cada sección donde se impongan 
condiciones de contorno, si es conocido el desplazamiento en una determinada dirección, el esfuerzo en esa misma dirección será una incógnita del problema y viceversa. Para un problema plano, toda la casuística posible relativa a la imposición de condiciones de contorno se puede resumir como sigue, por ejemplo, en el extremo $(s=L)$, relativas al sistema de referencia local de la barra, se puede expresar como:

$$
\left[\begin{array}{c}
N_{s}(L) \\
V_{y}(L) \\
M_{z}(L)
\end{array}\right]=-\left[\begin{array}{ccc}
k_{u} & 0 & 0 \\
0 & k_{v} & 0 \\
0 & 0 & k_{\theta}
\end{array}\right] \cdot\left[\begin{array}{c}
u(L) \\
v(L) \\
\theta(L)
\end{array}\right]
$$

Esta representación de las condiciones de contorno, incluye entre otras, las más habituales: apoyo rígido $\left(k_{i}=\infty\right)$, extremo libre $\left(k_{i}=0\right)$, apoyos elásticos $\left(0<k_{i}<\infty\right)$, etc.

El siguiente paso será resolver el conjunto de ecuaciones $(2.7) /(2.14)$ y $(2.15)$ de todas las barras de la estructura junto con las condiciones de equilibrio y compatibilidad en los nudos, y de contorno en los apoyos para determinar la respuesta de la estructura.

\subsubsection{Análisis límite}

Prácticamente siempre los primeros análisis que se llevan a cabo en cualquier diseño estructural son análisis estáticos con el objetivo de determinar el máximo nivel de carga $\left(\lambda_{E}\right)$ para el cual ninguna fibra del material que constituye las barras del pórtico plastifique, ya se plantee el equilibrio en la configuración inicial (análisis lineal) o en la configuración actual (análisis no lineal).

Después de completar el diseño elástico es muy conveniente realizar el análisis límite o cálculo plástico de la estructura con un doble objetivo: primero, y más habitual, que dicho cálculo nos sirva para evaluar la reserva de resistencia con la que cuenta la estructura frente a situaciones accidentales, y evaluar de esta forma el coeficiente de seguridad del diseño $(n)$; segundo, pasar a realizar un diseño plástico, es decir, dimensionar la estructura de forma que sea estable y segura con el ahorro de material que supone dicho modelo de análisis. En cualquiera de los dos escenarios es muy importante calcular con la suficiente precisión la carga de colapso $\left(\lambda_{u}\right)$, el mecanismo de colapso, y la respuesta del pórtico, esfuerzos, desplazamientos y giros, y resto de magnitudes de interés en el estado último o límite del conjunto de barras. 
El método de análisis utilizado es el desarrollado en este trabajo, se trata de un método paso a paso implícito debido al modelo barra 2D utilizado. Incremental de paso variable para el análisis en configuración inicial, e incremental-iterativo (algoritmo de Newton-Raphson) en el caso de planteamiento del equilibro en configuración actual.

\subsubsection{Estabilidad}

Según la teoría de la Resistencia de Materiales la solución de las ecuaciones (2.14) y (2.15) para unas determinadas condiciones de contorno es única. Sin embargo, para ciertos valores discretos del factor de carga, podría no ser así, lo que significa que puede producirse una deflexión súbita de amplitud indeterminada pero de forma conocida (o predecible) denominada modo de pandeo.

Para determinar el nivel crítico de carga asociado a dicha inestabilidad, existe un método de cálculo basado en introducir una pequeña perturbación al sistema que consiste en suponer un desplazamiento transversal infinitesimal $\Delta \underline{u}$ respecto al estado de equilibrio estable. En esta nueva situación se deben seguir cumpliendo las ecuaciones de equilibrio (2.14), comportamiento y compatibilidad (2.15). De manera que las magnitudes incrementales en desplazamientos $(\Delta u, \Delta v, \Delta \theta)$ y esfuerzos $(\Delta H, \Delta V, \Delta M)$ deben satisfacer las que se conocen como ecuaciones de estabilidad ${ }^{43}$ :

$$
\left\{\begin{array}{l}
\Delta H^{\prime}(s)=0 \\
\Delta V^{\prime}(s)=0 \\
\Delta M^{\prime}(s)-H(s) \Delta \theta(s)+\Delta V(s)=0
\end{array}\right.
$$

siendo $(H(s))$ el esfuerzo axil acumulado en cada barra durante el proceso de carga.

Sistema de ecuaciones diferenciales con las mismas condiciones de sustentación que el problema original y cargas exteriores todas ellas nulas, lo que desde el punto de vista matemático supone un problema de valor frontera cuyos autovalores son los valores del factor de carga crítico de interés.

La deformada en el instante de pandeo resulta indeterminada y por tanto, determinar el modo de pandeo asociado requiere resolver las ecuaciones de estabilidad (2.32) para el nivel crítico de carga mediante la sustitución de una condición de equilibrio por una condición de contorno adicional en desplazamientos, o dicho de otra forma imponiendo un 
valor arbitrario para alguno de los grados de libertad de la estructura objeto de análisis.

\subsubsection{Comportamiento vibratorio}

Un análisis dinámico presenta una mayor complejidad que un análisis estático debido a la naturaleza variable de la excitación, una carga dinámica se caracteriza porque su magnitud, dirección y/o punto de aplicación varía con el tiempo, y porque la velocidad y aceleración de los puntos materiales (inercia) intervienen en las ecuaciones de equilibrio. La respuesta puede ser un movimiento periódico alrededor de la posición de equilibrio, las oscilaciones se prolongan en el tiempo mediante un proceso de conversión entre los distintos tipos de energía, si en dicho proceso interviene la energía de deformación la oscilación puede alcanzar la categoría de vibración. En cada ciclo de vibración, el sistema disipa una cierta cantidad de energía con lo que los períodos decrecen según avanza el tiempo, es decir, el sistema real es amortiguado.

Los problemas dinámicos más habituales en la práctica suelen ser verificaciones de diseños mediante el análisis teórico y/o el testeo experimental. Para realizar un análisis teórico hay que: crear el modelo matemático (consiste en idealizar el sistema físico real a través de hipótesis simplificativas que permiten obtener un modelo aproximado del real, más sencillo de analizar); obtener las ecuaciones de equilibrio (de la aplicación de la dinámica al modelo matemático se obtienen las ecuaciones diferenciales que gobiernan el movimiento del sistema); resolución de las ecuaciones del movimiento (se trata de obtener la ley de desplazamientos del sistema en función del tiempo, para ello se utilizan métodos analíticos o numéricos); e interpretación de los resultados (el ingeniero a la vista de los resultados, valida el modelo matemático o por el contrario lo modifica).

Los métodos que se utilizan para plantear las ecuaciones de la dinámica son: los teoremas de la cantidad de movimiento y del momento cinético derivados de la segunda Ley de Newton, el principio de D 'Alembert, el principio de los trabajos virtuales, el teorema de la energía o las ecuaciones de Lagrange. Y así en configuración inicial resulta:

$$
\left\{\begin{array}{l}
\Delta N_{s}^{\prime}(s, t)=\rho A \Delta \ddot{u}(s, t) \\
\Delta V_{y}^{\prime}(s, t)=\rho A \Delta \ddot{v}(s, t) \\
\Delta M_{z}^{\prime}(s, t)+V_{y}(s, t)=\rho I_{z} \Delta \ddot{\theta}(s, t)
\end{array}\right.
$$


donde la notación $\left({ }^{*}\right)$ indica derivada temporal de una variable.

La solución de las vibraciones libres no amortiguadas es una función armónica de frecuencia $\omega_{n}$, la cual depende de los parámetros mecánicos del sistema (rigideces y masa inercial) pero no depende del tiempo ni de las condiciones iniciales. Las frecuencias naturales o de vibración son características intrínsecas de la estructura que define, al menos en parte, su comportamiento vibratorio. Así, cuando una de estas frecuencias coincide con la frecuencia de la fuerza excitadora, ocurre el fenómeno conocido como resonancia, el cual conlleva una amplificación de los desplazamientos que puede originar, en función del amortiguamiento y de otras propiedades del material, el colapso de la estructura.

Es una práctica habitual la correlación de los resultados entre el modelo matemático y el real; es decir, comparar los resultados obtenidos mediante el análisis experimental, y en consecuencia hacer las modificaciones pertinentes al modelo teórico hasta alcanzar un determinado grado de ajuste entre ambos. Debe tenerse en cuenta que si las medidas están bien hechas, es el modelo experimental el que manda. Esto puede llevar a preguntarse el porqué del análisis teórico, la primera de las razones es el coste económico, un análisis experimental es mucho más caro. En segundo lugar, si se trata de un diseño, una vez encontrado un modelo que se ajuste adecuadamente a la realidad, cualquier modificación y análisis posterior a partir del mismo es mucho más barato y sencillo que tener que construir prototipos y analizarlos experimentalmente. El Análisis Experimental de Vibraciones (AEV) emplea excitaciones de tipo impulso, así como sus sucesivas integrales (funciones escalón y rampa) para determinar la propiedades dinámicas del sistema.

A continuación, se propone una solución de variables separadas para la vibración transversal $(\Delta v(s, t)=\Delta v(s) \cdot T(t))$, supuesto despreciable la inercia rotacional, sistema de ecuaciones espacial que ahora en configuración actual es:

$$
\left\{\begin{array}{l}
\Delta N_{s}^{\prime}(s)=0 \\
\Delta V_{y}^{\prime}(s)+\rho A \omega_{n}^{2} \Delta v(s)=0 \\
\Delta M_{z}^{\prime}(s)-H(s) \Delta \theta(s)+V_{y}(s)=0
\end{array}\right.
$$

que junto con las condiciones de contorno constituye el problema de autovalores a resolver para determinar $\left(\omega_{n}\right)$, la frecuencia natural o propia del sistema.

Por último, recordar que como es bien sabido, dependiendo de las condiciones iniciales, 
la respuesta total tiene una contribución distinta de cada modo, es decir, se excitan de distinta forma unos modos $\mathrm{u}$ otros.

\subsubsection{Optimización}

El cálculo de la carga y el mecanismo de colapso mediante un elemento barra con comportamiento elástico lineal en el dominio y comportamiento plástico localizado en las secciones extremas, modelizado mediante nudos semirrígidos como el indicado líneas arriba en la figura 2.3, permite analizar de forma sencilla sistemas de barras rectas no prismáticas, es decir, conjuntos de barras de propiedades estáticas (canto, área e inercia) variables a lo largo de la longitud de cada una de las barras que forman el pórtico plano. Este proceso de cálculo se lleva a cabo con técnicas numéricas avanzadas, ya que en general, no tiene solución matemática cerrada, pero presenta la gran ventaja (respecto al método matricial de rigidez, el más empleado en la práctica) de que no requiere deducir la matriz de rigidez para cada tipo de variación del perfil, ni requiere ningún tipo de expresión explícita del efecto en los nudos de las cargas aplicadas en el interior de cada elemento barra, lo que habitualmente se conoce con el término de fuerzas de empotramiento, vectores de fuerzas recogidos ampliamente en la literatura para los casos más habituales de carga para el caso lineal, no así para el planteamiento del equilibrio en la configuración actual ni para el caso de no linealidad material con plasticidad acoplada. Así mismo, durante el proceso de carga, el método propuesto en este trabajo comprueba la estabilidad de la estructura y calcula sus primeras frecuencias propias de vibración, así como sus modos correspondientes.

El hecho de contar con una herramienta de cálculo que permite analizar estructuras planas con barras de perfil variable solicitadas por cualquier tipo de carga: fuerzas puntuales, momentos concentrados, carga distribuida de cualquier tipo e incluso carga de origen térmico (lógicamente, peso propio y efecto de la temperatura no ponderados por el factor de carga), nos lleva a aprovechar dicha formulación para plantear y resolver los problemas de optimización que resulten de interés. Para ello, se elige un conjunto de parámetros que definan la variación del canto a lo largo de la longitud de cada barra y se plantea la optimización de forma del pórtico.

Se pueden plantear distintos problemas de optimización, según la función objetivo elegida, es decir, magnitud de la respuesta del pórtico a maximizar o minimizar, se puede 
buscar maximizar la estabilidad (maximizar el valor de la carga crítica) o minimizar la cantidad de material empleado (minimizar volumen de material) por ejemplo, sujeto a algunas o todas de las restricciones siguientes: resistencia, volumen de material dado, desplazamientos y deformaciones admisibles, valor mínimo establecido de la carga crítica, valores máximos o mínimos (según interese) de las primeras frecuencias propias, etc.

Así por ejemplo, a partir de estas ideas y en base a la metodología expuesta en este trabajo de Tesis, un artículo de investigación recientemente aceptado para su publicación, se centra concretamente en optimizar la forma geométrica de pórticos con el objetivo de maximizar la carga crítica asociada al fenómeno de pandeo por flexión sujeto a las restricciones de volumen de material limitado y tensiones máximas equivalentes en cualquier punto inferiores al límite de fluencia del material. Tanto la función objetivo, como las restricciones, en general son no lineales, motivo por el que se ha elegido como técnica de optimización un método de Programación No Lineal, concretamente el algoritmo de Programación Cuadrática Sucesiva (SQP - Sequential Quadratic Programming ${ }^{27,35}$ ) junto con la herramienta de computación técnica MATLAB ${ }^{\circledR}{ }^{9,16}$. Además, el código de programación se ha desarrollado de forma que la elección de variables resulte sencilla y versátil, con posibilidad de modificar la función objetivo, y por último, de manera que sea muy simple tanto eliminar como añadir nuevas restricciones al problema. 
3

\section{Resultados y Discusión}

3.1. Ejemplo 1: Viga apoyada-empotrada . . . . . . . . 38

3.1.1. Métodos clásicos . . . . . . . . . . . . . . . . . . . . . 39

3.1.2. Método propuesto . . . . . . . . . . . . . . 43

3.2. Ejemplo 2: Pórtico de Lee . . . . . . . . . . . . 54

3.3. Ejemplo 3: Pórtico simple a dos aguas . . . . . . . . . 56 
Como aplicaciones a casos concretos se presentan seguidamente tres ejemplos. En el primero se compara la carga de colapso y otras magnitudes obtenidas utilizando el método propuesto y dos métodos clásicos. Se realiza un seguimiento a medida que aumenta la carga hasta el colapso utilizando la función de plastificación $\left(Y_{M}\right)$ en configuración inicial e $\left(Y_{M N}\right)$ en configuración actualizada. El objetivo es presentar ejemplos de complejidad creciente con los que se pretende mostrar la metodología de cálculo expuesta en el capítulo anterior así como realizar una comparación de los resultados entre los distintos modelos de cálculo.

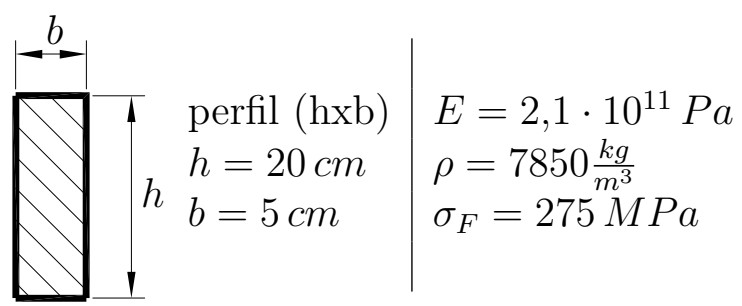

Tabla 3.1: Ejemplos. Datos comunes

\subsection{Ejemplo 1: Viga apoyada-empotrada}

En este ejemplo, a modo de validación, se resuelve el problema de una barra apoyadaempotrada sometida a una carga de compresión $(P)$ y a carga distribuida transversal $(q)$, tal y como se indica en la figura 3.1.

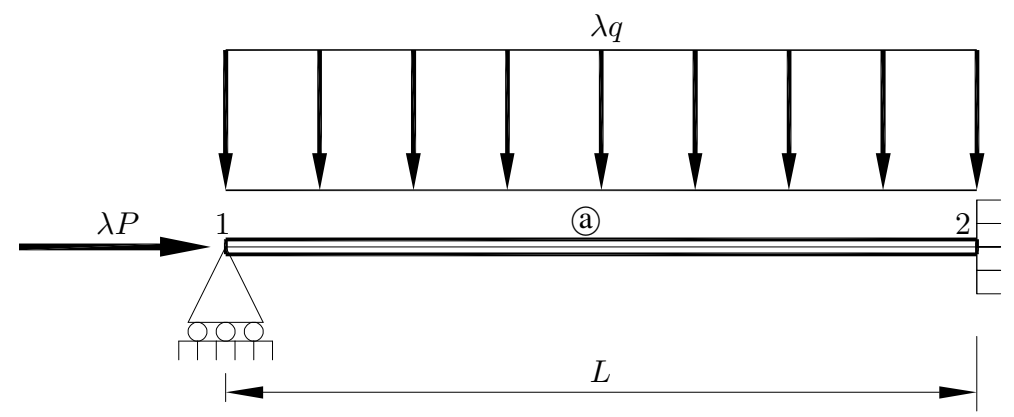

Figura 3.1: Viga apoyada-empotrada. 


\subsubsection{Métodos clásicos}

Los métodos clásicos de análisis para calcular la respuesta de la estructura en el colapso están basados en la hipótesis de la relación momento-curvatura $(M-\kappa)$ ideal, la estructura en el instante de colapso se supone formada por regiones (porciones de barra) perfectamente elásticas separadas entre sí por rótulas plásticas concentradas. En la actualidad, existen dos categorías de métodos para llevar a cabo dicho análisis que son los métodos paso a paso y los métodos directos.

\section{- Método paso a paso}

Los métodos paso a paso consisten en resolver la sucesión de problemas elásticolineales que resultan de la modificación de la estática de la estructura. A continuación, a modo de ejemplo, y con ánimo de comparar los resultados con los que se presentarán más adelante bajo otras hipótesis vamos a aplicar un método paso a paso para la viga de la figura 3.1.

Paso 1: consiste en resolver la estructura mediante algún método de análisis lineal como puede ser en el caso más general, el Método Directo de Rigidez, o bien, para un cálculo simple el Método de Compatibilidad o el Método de Slope-Deflection. Para este caso concreto, la estructura a analizar es muy sencilla, se trata de una estructura hiperestática de grado 1, por lo que se opta por resolverla mediante el Método de Compatibilidad.

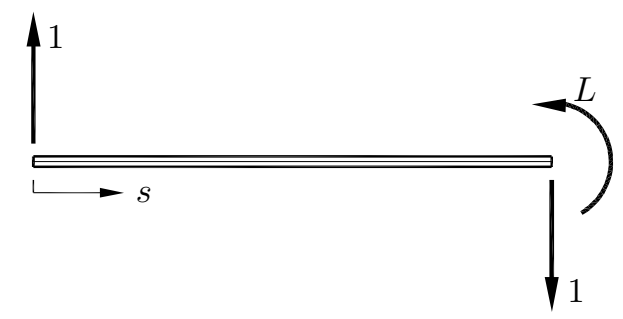

Figura 3.2: Viga. Problema virtual/auxiliar en esfuerzos (PFV)

Según este método de cálculo, el primer paso consiste en determinar los esfuerzos internos de la estructura en función de las reacciones (solo una, por ser hiperestática de grado uno, y elegimos $F$ en este caso, reacción vertical en el apoyo izquierdo) y 
proponer un problema auxiliar en equilibrio (problema virtual, figura 3.2):

$$
\left\{\begin{array} { l } 
{ N _ { s 1 } ( s ) = - P } \\
{ V _ { y 1 } ( s ) = q s - F } \\
{ M _ { z 1 } ( s ) = F s - \frac { s ^ { 2 } } { 2 } }
\end{array} \quad \left\{\begin{array}{l}
n_{s}(s)=0 \\
v_{y}(s)=-1 \\
m_{z}(s)=s
\end{array}\right.\right.
$$

donde las magnitudes $\left(N_{s}(s), V_{y}(s), M_{z}(s)\right)$ son los esfuerzos del problema real y $\left(n_{s}(s), v_{y}(s), m_{z}(s)\right)$ son los esfuerzos del problema virtual auxiliar.

El problema auxiliar en equilibrio de la figura 3.2 permite formular la ecuación de compatibilidad necesaria, mediante el Principio de la Fuerzas Virtuales (PFV) para obtener la incógnita hiperestática, en nuestro caso, la reacción vertical en el apoyo de la izquierda:

$$
\int_{0}^{L} M_{z 1}(s) \frac{m_{z}(s)}{E I_{z}} d s=0 ; \quad F=\frac{3}{8} q L
$$

como es habitual se ha despreciado la deformación debida al cortante.

Conocida la reacción vertical en el nudo (1), mediante equilibrio se puede calcular las leyes y los diagramas de esfuerzos de la estructura, y se comprueba que la sección más solicitada es la sección (2), por lo tanto, según vaya aumentando la carga, en esta sección se formará la primera rótula plástica:

$$
M_{\text {max }}=\frac{q L^{2}}{8}=M_{P} ; \quad q_{1}=\frac{8 M_{P}}{L^{2}}
$$

Paso 2: consiste en analizar la estructura con la libertad que introduce la formación de la rótula plástica en la sección (2). Problema al que corresponde la siguiente ley de momentos flectores:

$$
\Delta M_{z}(s)=\frac{1}{2} q(L-s) s
$$

Para localizar la formación de la siguiente rótula plástica debemos calcular el valor 
máximo de la distribución total/acumulada de momentos flectores:

$$
\begin{aligned}
& M_{z 2}(s)=M_{z 1}(s)+\Delta M_{z}(s) \\
& \frac{\partial M_{z 2}(s)}{\partial s}=0 ; \quad s=\frac{L\left(6 M_{P}+q L^{2}\right)}{2\left(8 M_{P}+q L^{2}\right)} \\
& M_{\max }=M_{P} ; \quad q_{2}=\frac{2 M_{P}(2 \sqrt{2}-1)}{L^{2}}
\end{aligned}
$$

Valor del incremento de carga que implica que se formará una rótula plástica en la sección situada en la cota:

$$
s_{2}=(\sqrt{2}-1) L
$$

Por lo tanto, la ley de momentos flectores incremental de la estructura en el instante en el que se forma la segunda rótula plástica tiene por expresión:

$$
\Delta M_{z}(s)=(2 \sqrt{2}-1) \frac{s(L-s)}{L^{2}} M_{P}
$$

Y el diagrama de momentos flectores en el instante de colapso se obtiene de representar sobre la viga los valores del esfuerzo flector total, es decir, de $\left(M_{z 2}(s)\right)$, suma de $\left(M_{z 1}(s)\right)$ y $\left(\Delta M_{z}(s)\right)$, distribución que coincide con la obtenida y dibujada en la figura 3.5. Entonces, de igual forma, acumulando, se deduce que la carga de colapso de la estructura es:

$$
q_{c}=q_{1}+q_{2}=\frac{2(3+2 \sqrt{2})}{L^{2}} M_{P}
$$

\section{- Método directo}

El método paso a paso presenta la ventaja de que permite seguir la evolución de la estructura hasta el colapso, pero puede suponer un esfuerzo operacional alto en estructuras con alto grado de hiperestaticidad. En algunas aplicaciones no es necesario conocer todo el proceso de plastificación al detalle sino que es suficiente con conocer la carga y el mecanismo de colapso.

Pues bien, existen métodos aproximados de aplicación simple para determinar la carga y el mecanismo de colapso, y que además permiten conocer la última rótula 
plástica que se forma, y los esfuerzos y desplazamientos en dicho instante, nos estamos refiriendo a los métodos directos.

El método directo, que vamos a aplicar a continuación, de análisis plástico de estructuras de barras está basado en los teoremas básicos del cálculo plástico: constancia de la curvatura, estático o de mínimo, cinemático o de máximo, y por último, el teorema de unicidad. Conjunto de teoremas que concluyen que el cálculo de la carga de colapso de una estructura se reduce a la búsqueda de una distribución de momentos flectores estáticamente admisible y segura que dé lugar a la formación de un mecanismo.

A continuación, se va a aplicar el método directo para comprobar la solución de este primer ejemplo (figura 3.1). Para dicho problema, las secciones candidatas a que en ellas se forme una rótula plástica son: la sección del apoyo empotrado, y una sección intermedia (posición concreta a determinar, $s_{2}$ ) debido a la carga distribuida aplicada. Con lo cual, es necesario aplicar una vez el Principio de los Desplazamientos Virtuales (PDV) para obtener la correspondiente ecuación de equilibrio (en vigas, habitualmente se considera únicamente la deformación asociada al momento flector), donde se toma como problema virtual auxiliar el campo de desplazamientos asociado al mecanismo indicado en la figura 3.3.

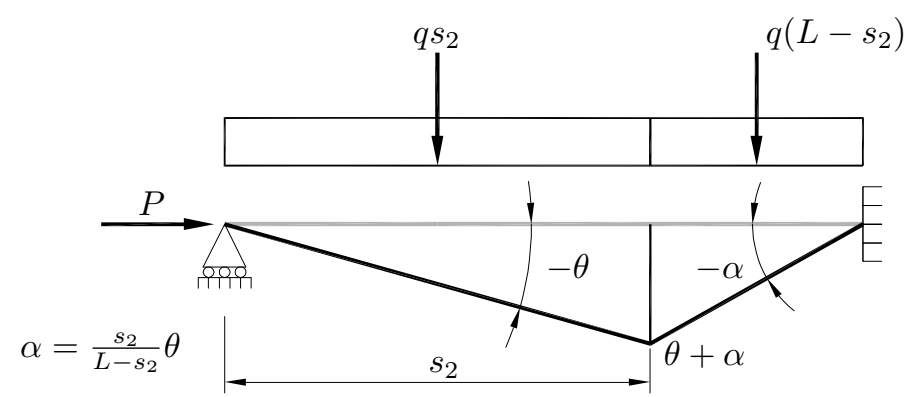

Figura 3.3: Viga. Problema virtual/auxiliar en desplazamientos (PDV)

$$
q s_{2} \frac{L}{2}=M_{z}\left(s_{2}\right) \frac{L}{L-s_{2}}-M_{z}(L) \frac{s_{2}}{L-s_{2}}
$$

Ahora, si ensayamos como mecanismo de colapso el propuesto anteriormente en la 
figura 3.3 para obtener la ecuación de equilibrio, tendremos:

$$
\left.\left.\begin{array}{l}
M_{z}\left(s_{2}\right)=+M_{P} \\
M_{z}(L)=-M_{P}
\end{array}\right\} \quad \begin{array}{l}
q_{c}=\frac{2 M_{P}}{s_{2} L} \frac{L+s_{2}}{L-s_{2}} \\
\frac{\partial q_{c}}{\partial s_{2}}=0 ; \quad s_{2}=(\sqrt{2}-1) L
\end{array}\right\} \quad q_{c}=\frac{2(3+2 \sqrt{2})}{L^{2}} M_{P}
$$

La ley del esfuerzo flector en el instante de colapso tiene por expresión:

$$
M_{z}(s)=\frac{(1+\sqrt{2})}{L^{2}} M_{P}(2 L-(1+\sqrt{2}) s) s
$$

solución que lógicamente coincide con la obtenida por el método incremental.

\subsubsection{Método propuesto}

Compararemos la solución anterior con aquellas obtenidas cuando se emplean modelos más sofisticados y realistas.

- Modelo 1: CI-RP- $Y_{M}$

Modelo clásico de cálculo plástico: planteamiento del equilibrio en la configuración inicial, modelo de rótula plástica concentrada súbita y plastificación de la sección únicamente por esfuerzo flector $\left(Y_{M}\right)$. Este modelo es el empleado en el apartado anterior.

- Modelo 2: CA-SA- $Y_{M N}$

Modelo avanzado de análisis límite: planteamiento del equilibrio en la configuración actual, plastificación completa de la sección con acoplamiento cinemático y estático a través de la función de plastificación $\left(Y_{M N}\right)$ que implica reducción del momento plástico por efecto del esfuerzo axil.

- Caso a: $L=4 m, P=10^{3} \mathrm{~N}, q=10^{3} \mathrm{~N} / \mathrm{m}$

Este primer caso corresponde a una viga poco esbelta donde el nivel de compresión es bajo, y predomina la carga y los esfuerzos de flexión.

En las tablas 3.2 y 3.3 se indican los resultados siguientes: el valor máximo del factor de carga $\left(\lambda_{E}\right)$, la carga crítica $\left(\lambda_{c r i}\right)$ y la primera frecuencia natural 


\begin{tabular}{|c|c|c|c|c|}
\hline \multirow[b]{3}{*}{$R P_{i}$} & \multicolumn{2}{|l|}{$\lambda_{E}$} & $\lambda_{\text {cri }}$ & $\omega_{0}(H z)$ \\
\hline & \multicolumn{2}{|l|}{45,0821} & 8833,44 & 45,7982 \\
\hline & \multicolumn{2}{|l|}{$\Delta \lambda$} & $\Delta \lambda_{c r i}$ & $\omega_{0, i}(H z)$ \\
\hline 1 & 68,7502 & $\mathrm{~L}$ & 4249,20 & 29,3166 \\
\hline 2 & 31,4259 & $0,414214 \cdot L$ & 19,0094 & 5,42032 \\
\hline$\lambda_{u}$ & 100,176 & $n=7$ & ${ }_{u} / \lambda_{E}=$ & \\
\hline
\end{tabular}

Tabla 3.2: Viga - 1.a.- Resultados - modelo 1 - caso a.

\begin{tabular}{|c|c|c|c|c|}
\hline \multirow[b]{3}{*}{$S A_{i}$} & \multicolumn{2}{|l|}{$\lambda_{E}$} & $\lambda_{c r i}$ & $\omega_{0}(H z)$ \\
\hline & \multicolumn{2}{|l|}{45,0784} & 8833,44 & 45,6838 \\
\hline & $\Delta \lambda$ & $s$ & $\Delta \lambda_{c r i}$ & $\overline{\omega_{0, i}(H z)}$ \\
\hline 1 & 68,3490 & $\mathrm{~L}$ & 4274,00 & 29,1671 \\
\hline 2 & 31,4282 & $0,414300 \cdot L$ & 34,498 & 2,9205 \\
\hline$\overline{\lambda_{u}}$ & 99,7772 & $n=$ & ${ }_{\iota} / \lambda_{E}=2$ & \\
\hline
\end{tabular}

Tabla 3.3: Viga - 2.a.- Resultados - modelo 2 - caso a.

$\left(\omega_{0}\right)$ según el diseño elástico; el incremento de carga $\left(\Delta \lambda_{i}\right)$ y la cota $(s)$ de la sección donde se produce la plastificación completa $(\mathrm{RP}$ - rótula plástica / SA - sección agotada), el incremento de carga crítica $\left(\Delta \lambda_{c r i}\right)$ y la primera frecuencia propia $\left(\omega_{0, i}\right)$ tras la modificación de la estática de la viga debido al proceso de plastificación; la carga última $\left(\lambda_{u}\right)$ de la estructura por formación de un mecanismo de colapso por plastificación o por pérdida de estabilidad y por último, el factor de seguridad $(n)$ del diseño no lineal respecto del elástico. La figura 3.4 muestra la deformada de la viga para un instante justo antes de que se forme la primera rótula plástica $\left(R P_{1}\right)$, línea de color azul, y la deformada un instante antes del colapso plástico, en color rojo $\left(R P_{2}\right)$.

En la figura 3.5 se comprueba la redistribución del esfuerzo momento flector que tiene lugar durante el proceso de plastificación, y así, se representa el diagrama correspondiente a dicho esfuerzo antes de la plastificación completa de la sección del empotramiento - línea de color azul - y justo antes de la formación de la rótula plástica interna - línea de color rojo - que da lugar al mecanismo de colapso.

La carga crítica y la frecuencia natural se obtienen en cada paso del proceso de carga como el menor de los valores propios del problema de valores de 


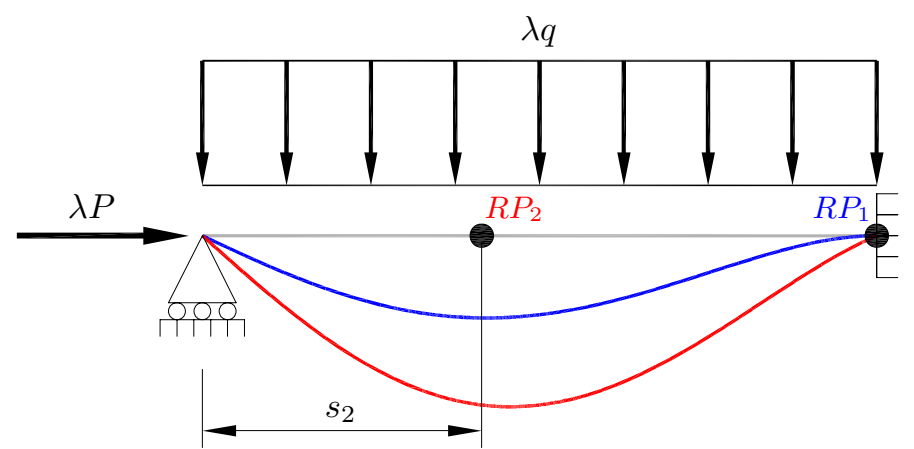

Figura 3.4: Viga - 1.a.- Deformada (amplificada x3600).

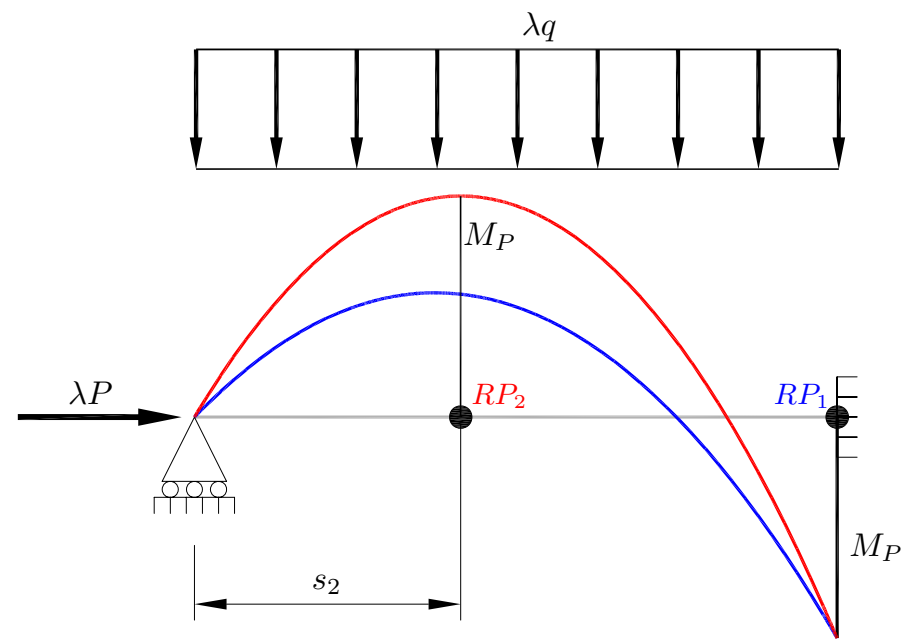

Figura 3.5: Viga - 1.a.- Diagrama de momento flector.

frontera descrito por el correspondiente sistema de ecuaciones diferenciales, es decir, se reduce a resolver un problema de autovalores. La figura 3.6 indica la variación del modo de pandeo y vibración durante el proceso de carga justo antes de que se produzca cada una de las rótulas plásticas.

Para este caso, comparando directamente ambas tablas, los resultados muestran que el cálculo plástico clásico, modelo de rótula plástica súbita concentrada, agotamiento de la sección por esfuerzo flector y planteamiento del equilibrio en la configuración inicial (lo que se ha denominado modelo 1, tabla 3.2) proporciona precisión suficiente.

- Caso b: $L=4 m, P=10^{4} \mathrm{~N}, q=10^{3} \mathrm{~N} / \mathrm{m}$

En este caso se incrementa el valor de la carga de compresión $(P)$ respecto a 


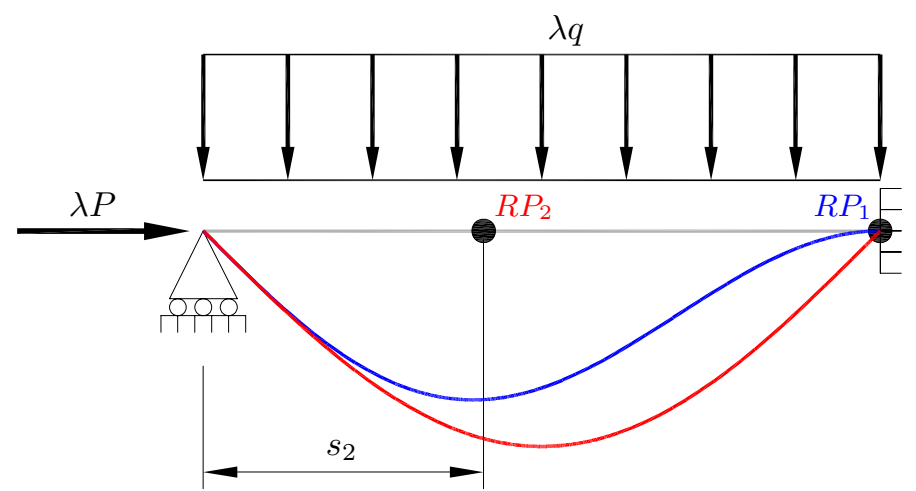

Figura 3.6: Viga - 1.a.- Modos de pandeo y vibración.

la carga distribuida $(q)$, los resultados son los que se indican en las tablas 3.4 y 3.5. Se observa la influencia del aumento del esfuerzo axil, y el efecto del acoplamiento estático debido a la curva de plastificación $\left(Y_{M N}\right)$ que implica una importante reducción de la capacidad máxima de momento flector de la sección. Y se comprueba una clara diferencia en el valor límite del factor de carga entre el método clásico (modelo 1, tabla 3.4) y el de sección agotada con momento plástico reducido $\left(M_{P N}\right)$ y equilibrio planteado en la configuración actualizada (modelo 2, tabla 3.5 ).

\begin{tabular}{|c|c|c|c|c|}
\hline \multirow[b]{3}{*}{$R P_{i}$} & \multicolumn{2}{|l|}{$\lambda_{E}$} & $\lambda_{c r i}$ & $\omega_{0}(H z)$ \\
\hline & \multicolumn{2}{|l|}{39,2858} & 883,344 & 45,7982 \\
\hline & $\Delta \lambda$ & $s$ & $\Delta \lambda_{c r i}$ & $\omega_{0, i}(H z)$ \\
\hline 1 & 68,7502 & $\mathrm{~L}$ & 363,045 & 29,3166 \\
\hline 2 & 31,4259 & $0,414214 \cdot L$ & 0,0 & 5,42032 \\
\hline$\lambda_{u}$ & 100,176 & $n=$ & $/ \lambda_{E}=2$ & \\
\hline
\end{tabular}

Tabla 3.4: Viga - 1.b.- Resultados - modelo 1 - caso b.

Las figuras 3.7 y 3.8 muestran la evolución del proceso de plastificación durante el proceso de carga para la sección del empotramiento - puntos de color rojo - y para la sección interna donde se produce la segunda plastificación completa - puntos de color azul -. La primera de ellas, la figura 3.7 muestra dicha evolución para el modelo 1, se observa claramente que hasta que se produce la primera rótula plástica el problema es lineal y después el problema incremental también (aunque la pendiente es distinta porque se modifica la rigidez 


\begin{tabular}{|c|c|c|c|c|}
\hline \multirow[b]{3}{*}{$S A_{i}$} & \multicolumn{2}{|l|}{$\lambda_{E}$} & $\lambda_{c r i}$ & $\omega_{0}(H z)$ \\
\hline & \multicolumn{2}{|l|}{39,2600} & 883,344 & 44,7912 \\
\hline & $\Delta \lambda$ & $s$ & $\Delta \lambda_{c r i}$ & $\omega_{0, i}(H z)$ \\
\hline 1 & 62,0983 & $\mathrm{~L}$ & 372,136 & 27,2162 \\
\hline 2 & 25,0555 & $0,417354 \cdot L$ & 0,0 & 0,0 \\
\hline$\lambda_{u}$ & 87,1538 & $n=$ & $/ \lambda_{E}=$ & \\
\hline
\end{tabular}

Tabla 3.5: Viga - 2.b.- Resultados - modelo 2 - caso b.

del sistema debido a la no linealidad material) por lo que no resulta necesario iterar. Sin embargo, para el modelo 2 (figura 3.8) es necesario emplear un método iterativo (algoritmo de Newton-Raphson, por ejemplo) para determinar la evolución de la respuesta de la estructura debido al planteamiento del equilibrio en la configuración actual, ya que hay una doble pérdida de rigidez de la viga, debido a la compresión y debido a la plastificación completa de la sección, efecto no lineal que es mucho más acusado cuanto mayor es el nivel de compresión de la barra.

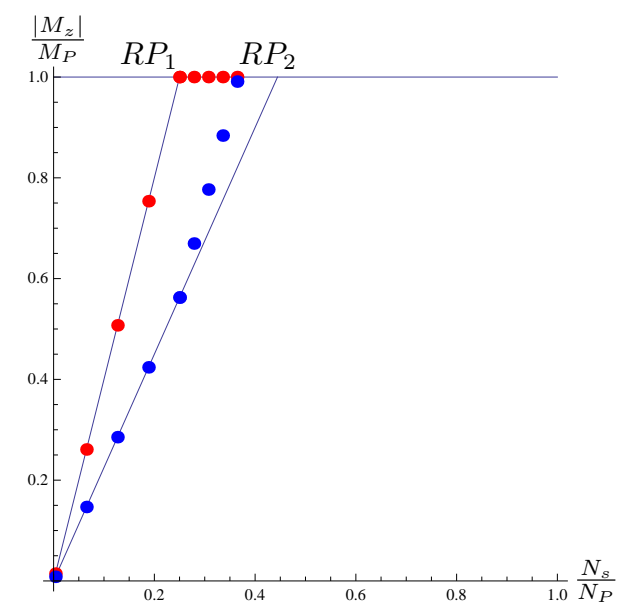

Figura 3.7: Viga - 1.b.- Evolución de la plastificación.

Por último, se muestra lo que creemos es la evolución de la primera frecuencia propia de la viga durante el proceso de carga (véanse las figuras 3.9 y 3.10). La primera de dichas figuras, la figura 3.9, representa la variación de la frecuencia natural durante el proceso de plastificación según el modelo 1, se observa una importante reducción del valor de la frecuencia cada vez que se modifica la 


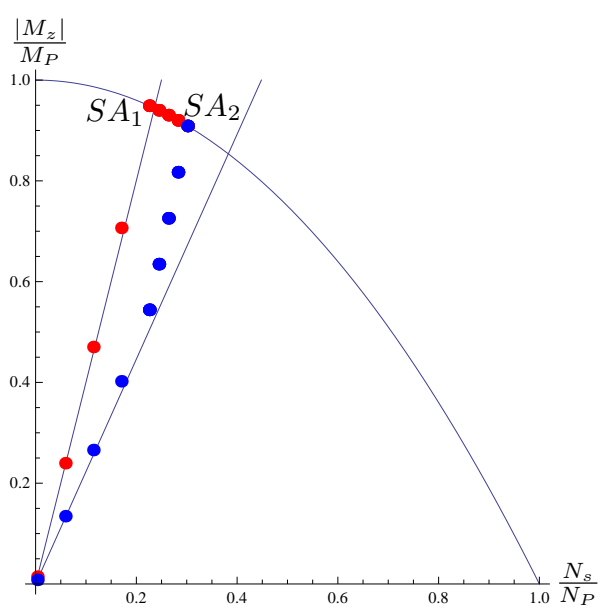

Figura 3.8: Viga - 2.b.- Evolución de la plastificación

estática de la estructura por la formación de una nueva rótula plástica, sin embargo, este modelo no permite captar la influencia del esfuerzo axil (de tracción o compresión). En la segunda figura, la figura 3.10, en el primer tramo, antes de que se forme la primera sección agotada se produce una leve reducción de la frecuencia propia, se observa la pérdida de rigidez de la viga exclusivamente debida a la compresión. La formación súbita de dicha sección agotada $\left(S A_{1}\right)$ produce una modificación repentina de la respuesta estática de la estructura que origina una importante reducción de la frecuencia y a partir de este instante el efecto del axil de compresión es más acusado hasta que se produce la segunda sección agotada $\left(S A_{2}\right)$, instante en que se forma el mecanismo de colapso por plastificación, al cual le corresponde un valor nulo de la primera frecuencia natural debido al hecho de que pueden producirse movimientos como sólido rígido, la estructura deja de comportarse como tal y pasa a ser un mecanismo, sin posibilidad de transmitir cargas por deformación.

- Caso c: $L=8 m, P=2 \cdot 10^{4} \mathrm{~N}, q=10^{2} \mathrm{~N} / \mathrm{m}$

Este caso particular considera una viga más esbelta, la longitud es el doble que para la barra de los dos casos anteriores, y se ha incrementado el valor de la carga de compresión $(P)$ respecto a la carga distribuida $(q)$ en mayor proporción que en el caso (b), de tal manera que predomine el efecto de la compresión. Los resultados para ambos modelos de análisis, modelo 1 y 2 


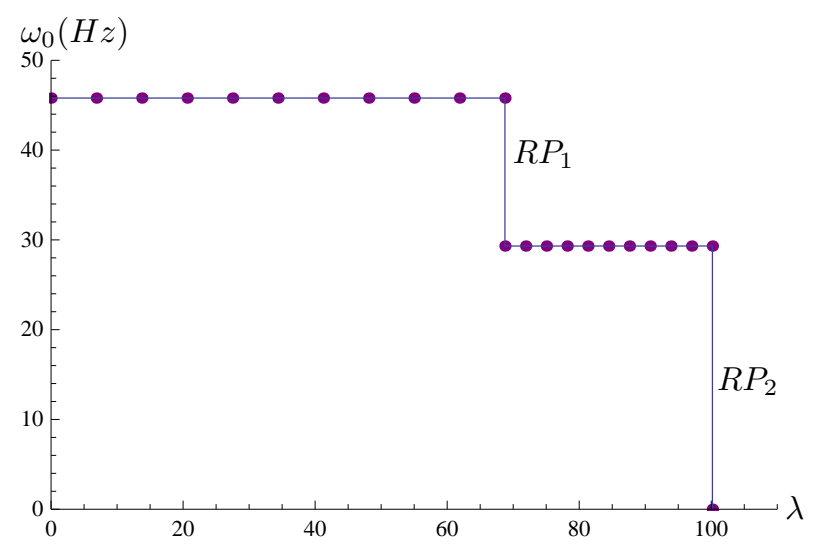

Figura 3.9: Viga - 1.b.- Evolución de la primera frecuencia propia

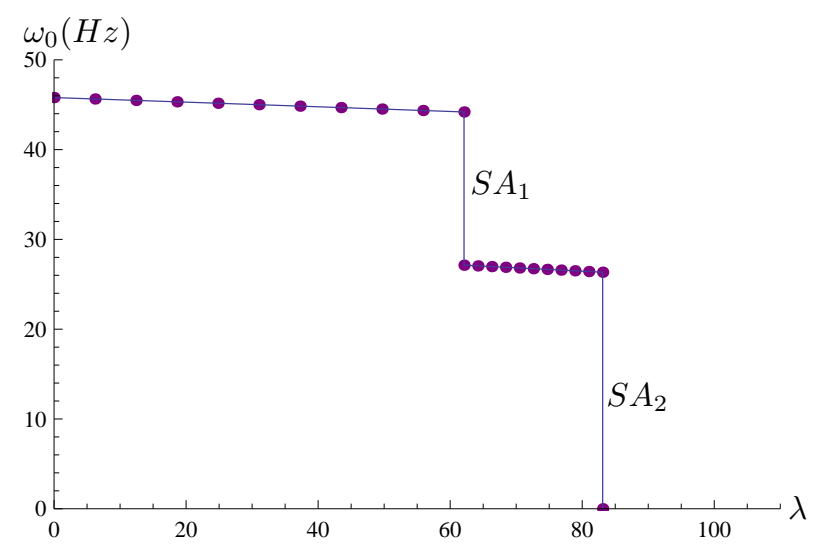

Figura 3.10: Viga - 2.b.- Evolución de la primera frecuencia propia

arriba indicados, se muestran en las tablas 3.6 y 3.7, respectivamente.

\begin{tabular}{|c|c|c|c|c|}
\hline \multirow[b]{3}{*}{$R P_{i}$} & \multicolumn{2}{|l|}{$\lambda_{E}$} & $\lambda_{c r i}$ & $\omega_{0}(H z)$ \\
\hline & \multicolumn{2}{|l|}{62,5001} & 110,418 & 11,4495 \\
\hline & $\Delta \lambda$ & $s$ & $\overline{\Delta \lambda_{c r i}}$ & $\omega_{0, i}(H z)$ \\
\hline 1 & 171,875 & $\mathrm{~L}$ & 0,0 & 7,32916 \\
\hline$\lambda_{u}$ & 110,418 & & $\lambda_{u} / \lambda_{1}$ & $=1,77$ \\
\hline
\end{tabular}

Tabla 3.6: Viga - 1.c.- Resultados - modelo 1 - caso c.

La segunda fila de dichas tablas presenta los resultados del análisis elástico y muestra una importante reducción de la frecuencia propia del análisis según el modelo $1(11,4495 \mathrm{~Hz})$ frente al modelo $2(7,61313 \mathrm{~Hz})$ debido a la com- 


\begin{tabular}{|c|c|c|c|c|}
\hline \multirow[b]{3}{*}{$S A_{i}$} & $\lambda_{E}$ & & $\lambda_{c r i}$ & $\omega_{0}(H z)$ \\
\hline & 62,2912 & & 110,418 & 7,61313 \\
\hline & $\Delta \lambda$ & $s$ & $\Delta \lambda_{c r i}$ & $\omega_{0, i}(H z)$ \\
\hline 1 & 66,5746 & $\mathrm{~L}$ & 0,0 & 0,0 \\
\hline$\lambda_{u}$ & 66,5746 & & $=\lambda_{u} / \lambda_{L}$ & $=1,07$ \\
\hline
\end{tabular}

Tabla 3.7: Viga - 2.c.- Resultados - modelo 2 - caso c.

presión a la que está sometida la barra, compresión que como es bien sabido produce una pérdida de rigidez del elemento y por lo tanto la correspondiente disminución del valor de la frecuencia natural del sistema, efecto que exige que el análisis incluya el planteamiento del equilibrio en la configuración deformada, motivo por el cual dicho efecto no es detectable con el modelo 1 que evalúa el equilibrio en configuración inicial.

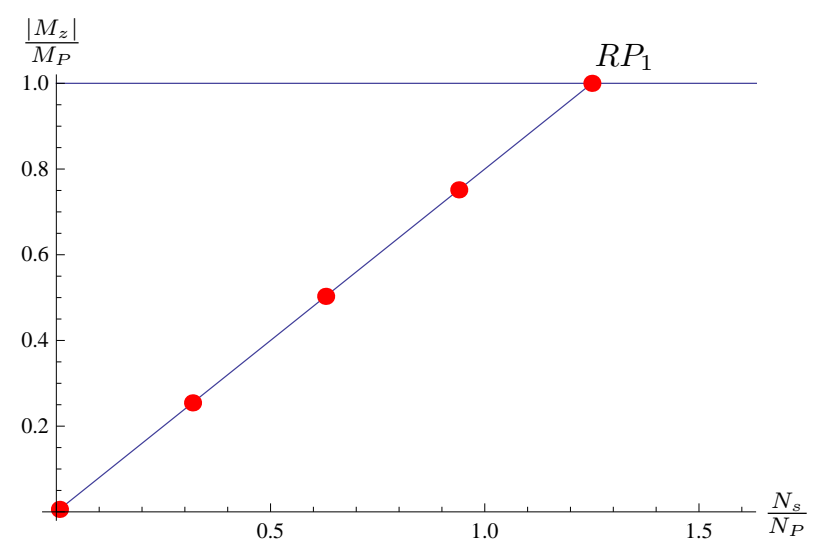

Figura 3.11: Viga - 1.c.- Evolución de la plastificación.

Respecto al proceso de plastificación (filas 4 y 5 de la tablas 3.6 y 3.7), un nivel alto de compresión y la consideración de su efecto en el equilibrio de momentos a través del planteamiento del equilibrio implica la formación de una única plastificación completa en la sección del empotramiento, después, se produce la inminente pérdida de estabilidad del conjunto de la estructura. En las figuras 3.11 y 3.12 se muestra la evolución de dicha sección según los modelos 1 y 2, respectivamente. Comparando ambas figuras destaca la no linealidad de la evolución de dichas secciones para el modelo 2 (véase la figura $3.12)$. 


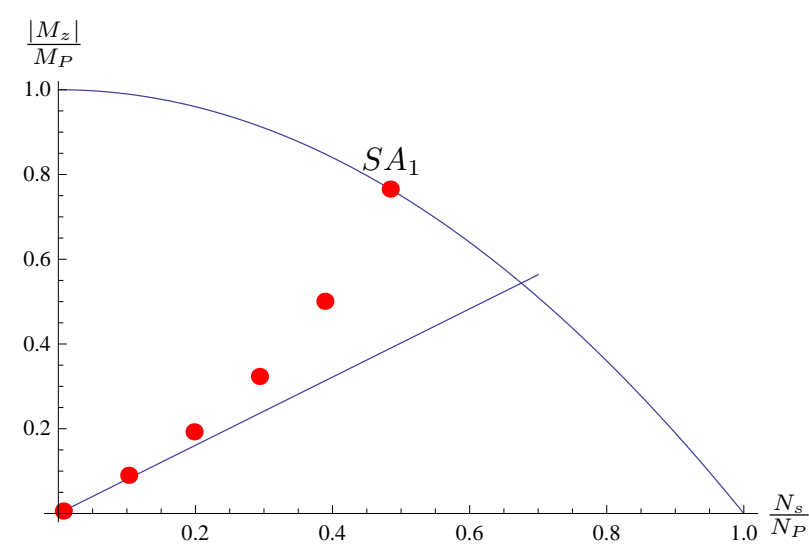

Figura 3.12: Viga - 2.c.- Evolución de la plastificación

Así, ambos modelos predicen que el colapso de la viga es debido a la pérdida de estabilidad tras la formación de la primera rótula plástica (modelo 1)/ sección agotada (modelo 2). En el estado último de la estructura, el modelo 1 (tabla 3.6) implica una reducción del valor de la frecuencia propia debida a la pérdida de rigidez que supone la modificación de la respuesta de la viga por la plastificación completa de la sección del empotramiento, pero que no tiene en cuenta la pérdida adicional por el efecto de la compresión que sí tiene en cuenta el modelo 2 (tabla 3.7) y que implica un valor nulo, resultado coherente con la formación de un mecanismo bien por plastificación o bien por pérdida de estabilidad como en este caso (véanse las figuras 3.13 y 3.14).

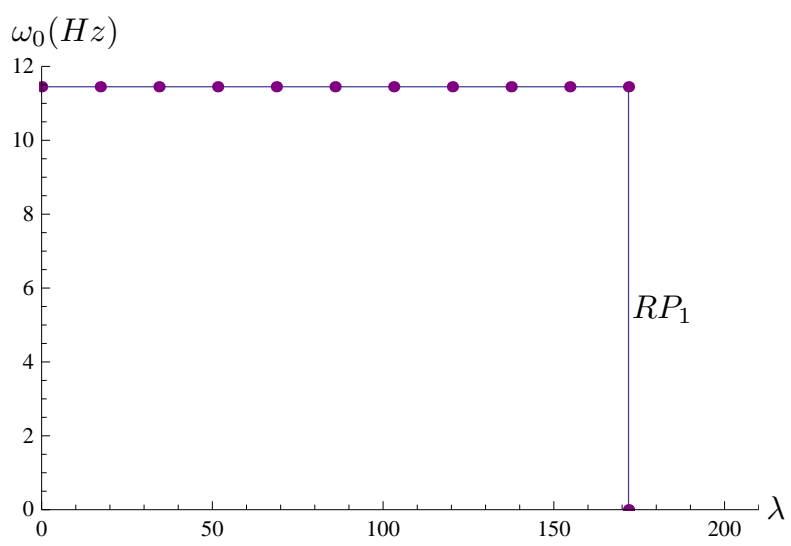

Figura 3.13: Viga - 1.c.- Evolución de la primera frecuencia propia

Como consecuencia de que en este caso el estado último de la estructura 


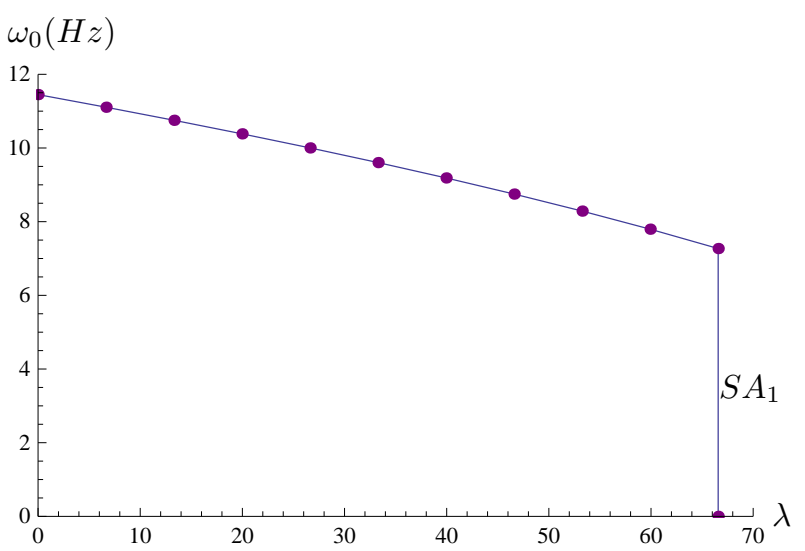

Figura 3.14: Viga - 2.c.- Evolución de la primera frecuencia propia

implica la pérdida de estabilidad global antes de la formación del mecanismo de colapso por proceso de plastificación, la carga última (110,418 según el modelo 1 y 66,5746 predice el modelo 2) es menor que la carga de colapso plástico. Incluso, en este caso, el modelo 2 predice un valor para el factor de seguridad del estado último (diseño no lineal estable) frente al diseño elástico muy próximo a la unidad.

- Caso d: $L=4 m, P=-10^{4} N$ (Tracción), $q=10^{3} \mathrm{~N} / \mathrm{m}$

Este último caso, es el caso (b) donde se ha cambiado el sentido de aplicación de la carga puntual horizontal $(P)$, para estudiar el efecto de un esfuerzo axil de tracción, los resultados para ambos modelos de estudio se reflejan en las tablas 3.8 y 3.9 , modelos 1 y 2 , respectivamente.

\begin{tabular}{|c|c|c|c|c|}
\hline \multirow[b]{3}{*}{$R P_{i}$} & \multicolumn{2}{|l|}{$\lambda_{E}$} & $\lambda_{c r i}$ & $\omega_{0}(H z)$ \\
\hline & \multicolumn{2}{|l|}{39,2858} & $-883,344$ & 45,7982 \\
\hline & $\Delta \lambda$ & $s$ & $\Delta \lambda_{c r i}$ & $\omega_{0, i}(H z)$ \\
\hline 1 & 68,7502 & $\mathrm{~L}$ & $-500,545$ & 29,3166 \\
\hline 2 & 31,4259 & $0,414214 \cdot L$ & $-112,095$ & 5,42032 \\
\hline$\lambda_{u}$ & 100,176 & $n=$ & $\lambda_{u} / \lambda_{E}=2$, & \\
\hline
\end{tabular}

Tabla 3.8: Viga - 1.d.- Resultados - modelo 1 - caso d.

Los resultados para el modelo 1 (tabla 3.8) coinciden exactamente con los recogidos en la tabla 3.4 del caso (b), como es lógico ya que dicho modelo 


\begin{tabular}{|c|c|c|c|c|}
\hline \multirow[b]{3}{*}{$S A_{i}$} & \multicolumn{2}{|l|}{$\lambda_{E}$} & $\lambda_{c r i}$ & $\omega_{0}(H z)$ \\
\hline & \multicolumn{2}{|l|}{39,3114} & $-883,344$ & 46,7833 \\
\hline & $\Delta \lambda$ & $s$ & $\Delta \lambda_{c r i}$ & $\omega_{0, i}(H z)$ \\
\hline 1 & 67,8380 & $\mathrm{~L}$ & $-502,073$ & 31,6125 \\
\hline 2 & 24,0866 & $0,410537 \cdot L$ & $-105,460$ & 16,0587 \\
\hline$\lambda_{u}$ & 91,9246 & $n=$ & $\lambda_{u} / \lambda_{E}=2$, & \\
\hline
\end{tabular}

Tabla 3.9: Viga - 2.d.- Resultados - modelo 2 - caso d.

no capta el efecto no lineal del esfuerzo axil con lo cual los resultados deben ser los mismos independientemente del valor y del sentido o signo de dicho esfuerzo.

Sin embargo, los resultados del modelo 2 (tabla 3.9) si que muestran diferencias respecto del caso (b) resuelto con el mismo modelo (tabla 3.5), diferencias del lado de la seguridad respecto al proceso de plastificación debido a la rigidización que produce un esfuerzo axil de tracción, y que como es lógico también conlleva un aumento en el valor de las frecuencias propias.

Por último, indicar que el valor negativo del factor de carga crítica implica que es necesario cambiar el sentido de aplicación de las cargas que solicitan la viga para que se produzca el fenómeno de inestabilidad de pandeo con deformaciones de flexión. 


\subsection{Ejemplo 2: Pórtico de Lee}

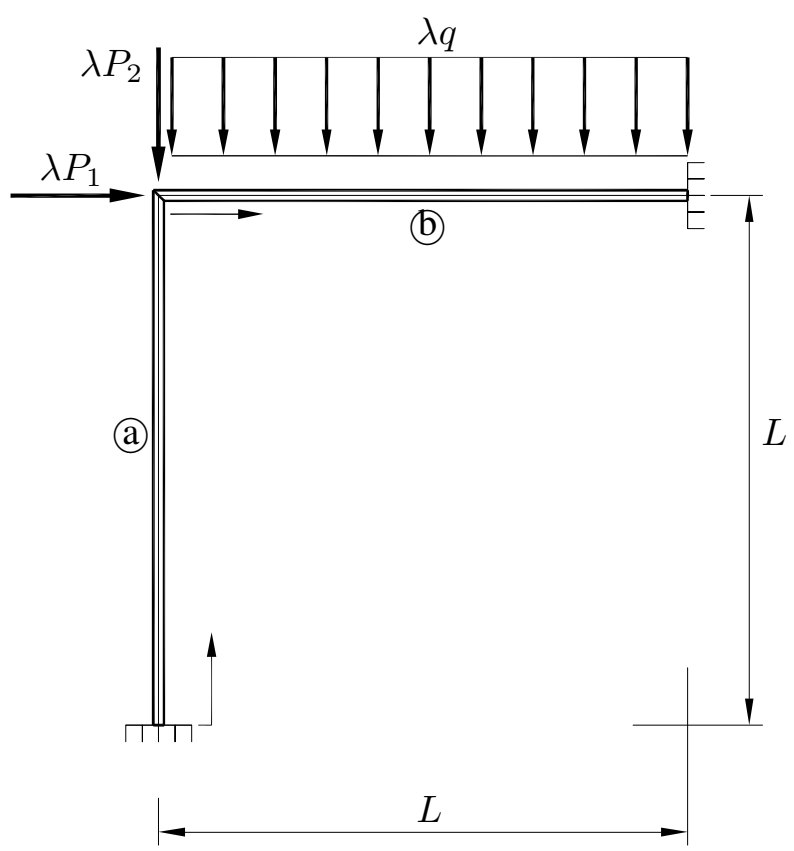

Figura 3.15: Pórtico de Lee

La estructura de la Figura 3.15, denominada en la literatura 'Pórtico de Lee'37, permite ilustrar de forma clara y sencilla el objetivo de este trabajo y las posibilidades de generalización de la técnica numérica empleada. Se considera por simplicidad que el pórtico está formado por barras iguales en cuanto a longitud $(L)$, material $\left(E, \rho, \alpha, \sigma_{F}\right)$ y perfil $\left(h, b, A, I_{z}\right)$, y se supone unión rígida pilar-dintel.

Se resuelven dos casos particulares para cada uno de los modelos indicados en el apartado anterior y los resultados se incluyen en las siguientes tablas: caso $a$, tablas 3.10 y 3.11 , y caso b, tablas 3.12 y 3.13 , para modelos 1 y 2 , respectivamente.

- Caso a: $L=4 m, P_{2}=P_{1}=10^{3} N, q=10^{2} N / m$

- Caso b: $L=4 m, P_{2}=P_{1}=10^{4} \mathrm{~N}, q=10^{2} \mathrm{~N} / \mathrm{m}$ 


\begin{tabular}{|c|c|c|c|c|c|}
\hline \multirow[b]{3}{*}{$R P_{i}$} & \multicolumn{3}{|l|}{$\lambda_{E}$} & $\lambda_{c r i}$ & $\omega_{0}(H z)$ \\
\hline & \multicolumn{3}{|l|}{444,059} & 8019,27 & 45,7982 \\
\hline & \multicolumn{2}{|l|}{$\Delta \lambda$} & $s$ & $\Delta \lambda_{c r i}$ & $\overline{\omega_{0, i}(H z)}$ \\
\hline 1 & 797,784 & (b) & $\mathrm{L}$ & 4397,98 & 34,2012 \\
\hline 2 & 488,767 & (b) & $0,483098 \cdot L$ & 0,0 & 17,3728 \\
\hline$\lambda_{u}$ & 1286,55 & & $n=\lambda_{u} /$ & $=2,90$ & \\
\hline
\end{tabular}

Tabla 3.10: Pórtico de Lee - 1.a.- Resultados - modelo 1 - caso a.

\begin{tabular}{|c|c|c|c|c|c|}
\hline \multirow[b]{3}{*}{$S A_{i}$} & \multicolumn{3}{|l|}{$\lambda_{E}$} & $\lambda_{c r i}$ & $\omega_{0}(H z)$ \\
\hline & \multicolumn{3}{|l|}{444,064} & 8019,22 & 45,5445 \\
\hline & \multicolumn{2}{|l|}{$\Delta \lambda$} & $s$ & $\Delta \lambda_{c r i}$ & $\overline{\omega_{0, i}(H z)}$ \\
\hline 1 & 709,563 & (b) & $\mathrm{L}$ & 4330,08 & 31,9206 \\
\hline 2 & 340,479 & (b) & $0,486419 \cdot L$ & 0,0 & 3,72088 \\
\hline$\lambda_{u}$ & 1050,04 & & $n=\lambda_{u} /$ & $E=2,36$ & \\
\hline
\end{tabular}

Tabla 3.11: Pórtico de Lee - 2.a.- Resultados - modelo 2 - caso a.

\begin{tabular}{|c|c|c|c|c|c|}
\hline \multirow[b]{3}{*}{$R P_{i}$} & \multicolumn{2}{|l|}{$\overline{\lambda_{E}}$} & & $\lambda_{c r i}$ & $\omega_{0}(H z)$ \\
\hline & \multicolumn{3}{|l|}{166,494} & 876,382 & 45,7982 \\
\hline & \multicolumn{2}{|l|}{$\Delta \lambda$} & $s$ & $\Delta \lambda_{c r i}$ & $\omega_{0, i}(H z)$ \\
\hline 1 & 632,936 & (b) & $\mathrm{L}$ & 0,0 & 34,2012 \\
\hline$\lambda_{u}$ & 632,936 & & & $\lambda_{u} / \lambda_{E}$ & 3,80 \\
\hline
\end{tabular}

Tabla 3.12: Pórtico de Lee - 1.b.- Resultados - modelo 1 - caso b.

\begin{tabular}{|c|c|c|c|c|c|}
\hline \multirow[b]{3}{*}{$S A_{i}$} & \multicolumn{3}{|l|}{$\lambda_{E}$} & $\lambda_{c r i}$ & $\omega_{0}(H z)$ \\
\hline & \multicolumn{3}{|l|}{166,475} & 876,376 & 41,3122 \\
\hline & \multicolumn{2}{|l|}{$\Delta \lambda$} & $s$ & $\Delta \lambda_{c r i}$ & $\omega_{0, i}(H z)$ \\
\hline 1 & 216,364 & (b) & $\mathrm{L}$ & 332,806 & 26,8613 \\
\hline 2 & 25,6792 & (b) & $0,467481 \cdot L$ & 0,0 & 0,0 \\
\hline$\lambda_{u}$ & 242,043 & & $n=\lambda_{u} /$ & $\lambda_{E}=1,45$ & \\
\hline
\end{tabular}

Tabla 3.13: Pórtico de Lee - 2.b.- Resultados - modelo 2 - caso b. 


\subsection{Ejemplo 3: Pórtico simple a dos aguas}

Se aplica finalmente la técnica de análisis al pórtico simple a dos aguas indicado en la Figura 3.16. Considerando una discretización de sólo cuatro elementos coincidentes con las barras que forman el pórtico y asumiendo un estado proporcional de cargas (siendo $\lambda$ dicho factor de proporcionalidad), resultan los valores indicados en las tablas 3.14 y 3.15 , modelos 1 y 2 , respectivamente, para el caso numérico: $L=4 m, \beta=15^{\circ}$, $q_{v}=q_{n}=100 \frac{N}{m}$.

\begin{tabular}{|l|c|c|c|r|r|}
\cline { 2 - 6 } \multicolumn{1}{c|}{} & \multicolumn{1}{c}{$\lambda_{E}$} & \multicolumn{3}{c|}{$\lambda_{\text {cri }}$} & $\omega_{0}(H z)$ \\
\cline { 2 - 6 } \multicolumn{1}{c}{$R P_{i}$} & 103,272 & \multicolumn{3}{c}{5737,43} & 11,5719 \\
\cline { 2 - 6 } \multicolumn{1}{c|}{$\Delta \lambda$} & \multicolumn{3}{c}{$\Delta \lambda_{\text {cri }}$} & $\omega_{0, i}(H z)$ \\
\hline 1 & 157,654 & d & $L_{d}$ & 3302,74 & 8,05947 \\
\cline { 2 - 6 } 2 & 55,3848 & C & $L_{c}$ & 1990,70 & 5,87435 \\
\cline { 2 - 6 } 3 & 24,1787 & (a) & 0 & 189,807 & 2,83838 \\
\cline { 2 - 6 } 4 & 21,7619 & (b) & $0,795203 \cdot L_{b}$ & 0,0 & 0,332274 \\
\hline$\lambda_{u}$ & 258,979 & \multicolumn{4}{c}{$n=\lambda_{u} / \lambda_{E}=2,51$} \\
\hline
\end{tabular}

Tabla 3.14: Pórtico simple a dos aguas.- Resultados - modelo 1.

\begin{tabular}{|c|c|c|c|c|c|}
\hline \multirow[b]{3}{*}{$S A_{i}$} & \multicolumn{3}{|l|}{$\lambda_{E}$} & \multirow{2}{*}{$\begin{array}{c}\lambda_{c r i} \\
5737.43\end{array}$} & $\omega_{0}(H z)$ \\
\hline & 103,263 & & & & 11,4713 \\
\hline & $\Delta \lambda$ & & $s$ & $\Delta \lambda_{c r i}$ & $\omega_{0, i}(H z)$ \\
\hline 1 & 155,222 & (d) & $L_{d}$ & 2590,60 & 7,87388 \\
\hline 2 & 56,4314 & (d) & 0 & 1282.04 & 5.51818 \\
\hline 3 & 23,8563 & (a) & 0 & 194,736 & 1,90835 \\
\hline 4 & 26,0272 & (c) & 0 & 0.0 & 0.0 \\
\hline$\lambda_{u}$ & 261,537 & & & $\lambda_{u} / \lambda_{E}=$ &, 53 \\
\hline
\end{tabular}

Tabla 3.15: Pórtico simple a dos aguas.- Resultados - modelo 2. 


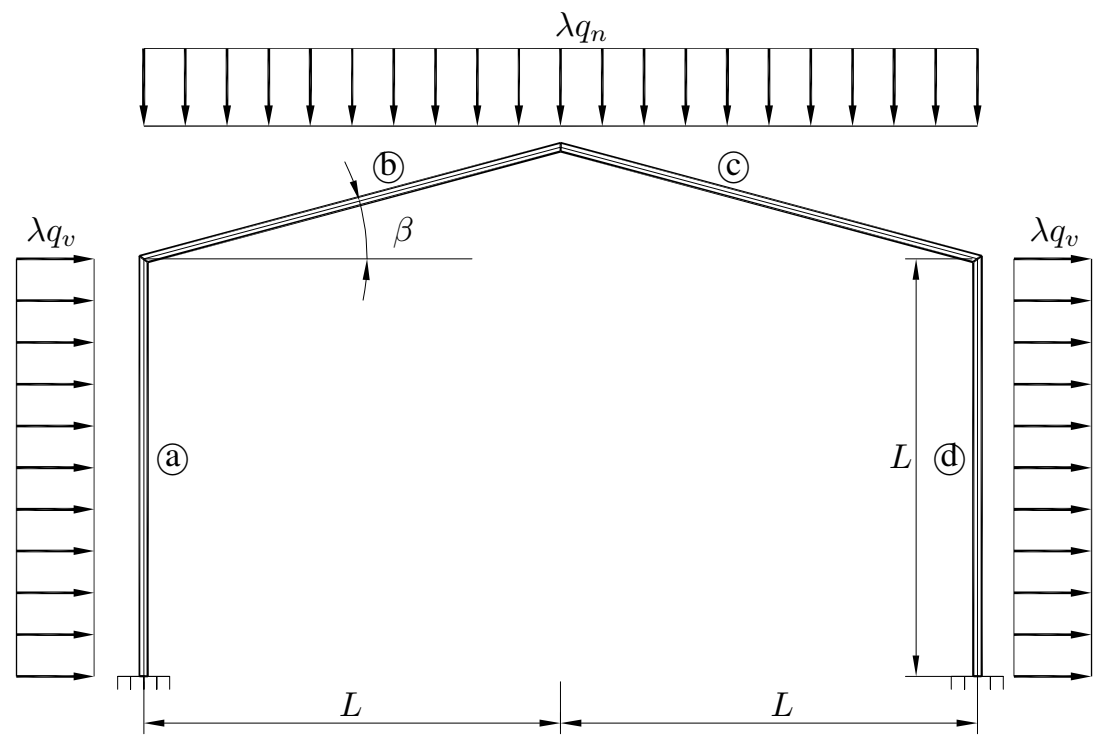

Figura 3.16: Pórtico simple a dos aguas 

4

Conclusiones 
A continuación se indican los alcances técnicos, ventajas e inconvenientes de: la metodología, la herramienta de análisis y de los resultados obtenidos, organizado por bloques de conocimiento:

1. Análisis límite/Cálculo plástico/No linealidad material. La ductilidad del material permite que las secciones sean sometidas a tensiones cada vez mayores hasta que finalmente se llega al colapso o agotamiento resistente. El comportamiento plástico, caracterizado por una deformación en parte irreversible, independiente del tiempo y dependiente de la historia de carga, se ha modelado en teoría de primer orden con un método paso a paso que considera plastificación sólo en función del momento flector y da lugar a la formulación de rótula plástica concentrada súbita, denominado modelo 1 en este documento. Concepto clásico de rótula plástica $(R P)$ que tiene su base en la consideración del momento flector como único esfuerzo que produce la plastificación, de forma que cuando su magnitud coincide con el momento plástico de la sección se considera que ésta ha plastificado de forma súbita y repentina.

También se estudia la aplicación de la Teoría de la Plasticidad al elemento barra 2D con no linealidad geométrica (teoría de segundo orden) y no linealidad material debido a comportamiento elastoplástico. Se consideran combinaciones de esfuerzos flector y axil, relacionados entre sí mediante la función de plastificación, el criterio de plastificación en este caso es que el momento flector ha de coincidir con el momento plástico reducido. Las distintas combinaciones de esfuerzos acoplados que producen la plastificación súbita de la sección originan desplazamientos relativos acoplados asociados a un único grado de libertad. Motivo por el cual en lugar de utilizar el concepto de rótula plástica, se emplea el concepto de sección agotada $(S A)$ por plastificación completa de la sección, que implica giro relativo y desplazamiento longitudinal relativo acoplados entre sí (referido en el documento como modelo 2).

La fluencia plástica del material produce pérdida de rigidez que afecta de manera considerable a las tensiones y deformaciones, y da lugar a una menor resistencia y capacidad portante de la sección y de la estructura en su conjunto. Por lo tanto, también se ve afectada la estabilidad parcial o total del conjunto, que se comprueba durante el proceso de carga cada vez que se produce plastificación completa de una 
nueva sección mediante el planteamiento de las ecuaciones de estabilidad, que como es bien sabido requiere plantear el equilibrio estático en la configuración deformada o actual.

Respecto a la metodología se plantea el equilibrio de cada barra en su configuración deformada, bajo hipótesis de pequeñas deformaciones y pequeños desplazamientos (teoría de segundo orden), resultando un sistema de ecuaciones diferenciales en general de coeficientes variables para cada barra. Para obtener la respuesta no lineal del conjunto es necesario imponer en cada unión compatibilidad de desplazamientos y equilibrio de fuerzas y momentos, nuevamente en la configuración deformada. Para llevar a cabo el análisis límite se formula un elemento barra 2D con comportamiento elástico lineal en el dominio y comportamiento plástico localizado en las secciones extremas modelizado mediante nudos semirrígidos.

Relativo a la herramienta de análisis desarrollada se pide que sea sistemática, y que permita de forma sencilla: incluir otros modelos teóricos, por ejemplo, grandes desplazamientos; cargas de cualquier tipo dentro del elemento; todo tipo de libertades en las uniones; cualquier tipo de apoyo, incluido apoyos elásticos, además de barras no prismáticas. Se trata de un método de cálculo que no se puede englobar dentro de los métodos de rigidez o de equilibrio ni como método de flexibilidad o compatibilidad, sino que es un método novedoso basado en la formulación diferencial a nivel de barra y en el cumplimiento riguroso de las condiciones de equilibrio y compatibilidad a nivel de estructura. Este planteamiento implícito tiene la ventaja de poder considerar sección transversal variable a lo largo de cada barra sin el requisito de los usuales métodos de análisis de calcular la matriz de rigidez o flexibilidad, y por lo tanto, sin la necesidad de actualizar dicha matriz para cada barra y para cada iteración, lo que supone un gran ahorro de cómputo, por ejemplo en procesos de optimización. Permite considerar cualquier tipo de carga, y por lo tanto incluir el efecto del peso propio y de la temperatura (ambos efectos, no afectados por el factor de carga).

Diseño plástico que da lugar a soluciones más racionales y una notable economía de los materiales, asegurando la seguridad, funcionalidad y durabilidad de la estructura.

Los resultados numéricos nos permiten comprobar que gracias a una formulación 
matemática rigurosa, la solución de desplazamientos para barra prismática se plantea en base a las funciones de estabilidad, planteamiento que presenta la ventaja de que al calcular se obtienen resultados con precisión suficiente incluso empleando discretizaciones groseras.

2. Inestabilidad/Pandeo De todas las condiciones de diseño de una estructura de barras, en muchos casos, la condición más crítica consiste en asegurar que bajo cualquier combinación posible de cargas no se produzca inestabilidad por flexión, más cuando la tendencia actual es diseñar estructuras esbeltas con aceros de mayor calidad. Resulta por tanto interesante contar con un método sistemático que permita determinar de forma directa la carga crítica y el modo de pandeo de cualquier pórtico plano sin necesidad de recurrir a las simplificaciones que usualmente se asumen en planteamientos matriciales o en el método de elementos finitos, lo que permite obtener resultados precisos con independencia de la discretización empleada.

En estabilidad, el punto de partida ha sido la teoría de pandeo de Euler, que asume pequeños desplazamientos y pequeñas deformaciones, y que da lugar a los conceptos de carga crítica y modo de pandeo, consecuencia del modelo matemático planteado. Por tanto, no es posible conocer los desplazamientos en el instante de pandeo (sólo $\mathrm{su}$ forma, lo que se conoce con el nombre de modo) ni el posible comportamiento post-pandeo.

Con el método propuesto, el cálculo de la respuesta de la estructura se hace muy sencillo y directo. Además, el planteamiento matemático del problema de inestabilidad como problema de autovalores a partir de las ecuaciones de estabilidad junto con las de equilibrio, compatibilidad-comportamiento y condiciones de contorno, permite obtener como resultado adicional del cálculo la carga crítica y el modo de pandeo con la misma metodología. 
3. Comportamiento Vibratorio. Además del análisis estático, la metodología incluye la capacidad de evaluar las frecuencias de vibración, así como los modos asociados. A este respecto, los resultados presentados son de carácter tentativo, lo que significa que deben ser corroborados por resultados experimentales ${ }^{*}$ o desarrollos teóricos futuros.

4. Optimización. Si se aprovecha la generalidad de la formulación desarrollada para incluir barras de sección variable bajo cualquier tipo de carga es posible analizar pórticos constituidos por barras no prismáticas y abordar problemas de optimización, y así por ejemplo buscar la variación óptima del canto de las barras de cualquier pórtico de manera que se cumplan cuantos criterios sean exigibles, entre ellos el de estabilidad, es decir, que no aparezcan fenómenos de pandeo. Para barras aisladas existen soluciones clásicas, en algunos casos analíticas, para la forma que debe tener una barra comprimida de manera que su resistencia al pandeo sea máxima. Pero para conjuntos/sistemas de barras el problema es más complejo y debe resolverse de forma numérica. En este sentido se presenta una formulación para resolver el problema de optimización de pórticos teniendo en cuenta los condicionantes no solo de estabilidad sino cualquier otro, como por ejemplo tensiones admisibles, desplazamientos limitados, etc . Para ello, se elige un conjunto de parámetros de diseño que definan la variación del canto a lo largo de la longitud de cada barra, y se formula matemáticamente el problema de optimización para determinar qué valores maximizan la carga de pandeo del pórtico sujeto a las restricciones de diseño (material, tensiones, desplazamientos, etc.), dando lugar a un sistema de ecuaciones diferenciales de coeficientes variables que se resuelve numéricamente mediante programación cuadrática secuencial.

En la práctica, es razonable pensar que todo problema de diseño se reduce siempre a un problema de optimización, en general con uno o más objetivos ponderados y con distintos tipos de restricciones: funcionalidad, seguridad, estabilidad, precio, estética, etc., lo que justifica el interés de este tipo de aportaciones y más hoy en día donde se tiende a abordar problemas de multifísica con equipos multidisciplinares.

\footnotetext{
*Adviértase que no es fácil comprobar experimentalmente los resultados presentados, al asumir ciertas hipótesis (rótula plástica concentrada y súbita, comportamiento elastoplástico perfecto, etc.) difícilmente reproducibles en laboratorio.
} 
La principal desventaja es la común de un método de optimización no lineal, sobre todo en lo relativo a la limitación en el número de parámetros para que los tiempos de cómputo no sean excesivos. De hecho en programación no lineal, en la actualidad, existe toda una línea de investigación con el objetivo de desarrollar técnicas numéricas orientadas a dar solución a problemas con gran número de parámetros. Algo que se está consiguiendo gracias al desarrollo de algoritmos cada vez más eficientes y al continuo avance de la capacidad de cálculo.

En la actualidad el grupo de investigación está trabajando en aplicar esta formulación a la optimización de forma de pórticos metálicos planos. Se busca el factor de seguridad máximo del diseño elástico y/o plástico bajo las restricciones habituales de cantidad de material limitado, desplazamientos admisibles, así como establecer restricciones adicionales relativas a fijar valores mínimos o máximos, según interese en la práctica, de las primeras frecuencias naturales o propias del conjunto estructural.

Todo ello persigue hacer un seguimiento de la respuesta de la estructura para cada estado de carga hasta el colapso, y así tener caracterizada la estructura tanto estática como dinámicamente. Algunas de estas magnitudes son fácilmente observable o medibles mediante técnicas experimentales, fundamentalmente el estado vibratorio. De esta manera se abre la posibilidad de aplicar el trabajo a técnicas de monitorizado y salud estructural, y de localización y evaluación de daño. 
5

Artículos publicados 
Artículos publicados:

a) CÁlculo directo de la CARGa CRítica De PANDEO DE PÓRTicos. PARTE I

A. Lorenzana y M. Cacho

Rev. Int. Mét. Num. Cálc. Dise. Ing., 25(3): 247-258, 2009.

b) Cálculo directo de la Carga Crítica de Pandeo DE PÓRTICOS. PARTE II

M. Cacho y A. Lorenzana

Rev. Int. Mét. Num. Cálc. Dise. Ing., 26: 31-38, 2010.

c) Optimización global de pórticos 2D CON BARRAs de SECCión VARIABle M. Cacho Pérez y A. Lorenzana Ibán DYNA Ingeniería e Industria. Aceptado: junio 2010.

Publicación, fecha prevista: noviembre 2010. 


\section{a) CÁlCULO DIRECTO DE LA CARGA CRÍTICA DE PANDEO DE PÓRTICOS. PARTE I}

A. Lorenzana y M. Cacho

Rev. Int. Mét. Num. Cálc. Dise. Ing., 25(3): 247-258, 2009.

\section{Revista Internacional de Métodos Numéricos} para Cálculo y Diseño en Ingeniería

Engineering, Multidisciplinary, Mathematics, Interdisciplinary Applications

\begin{tabular}{|c|c|c|c|c|c|c|c|c|c|}
\hline \multirow[b]{2}{*}{$\begin{array}{l}\text { Abbreviated Journal Title } \\
\text { (linked to journal information) }\end{array}$} & \multirow[b]{2}{*}{ ISSN } & \multicolumn{6}{|c|}{ JCR Data i) } & \multicolumn{2}{|c|}{ Eigenfactor $^{\mathrm{TM}}$ Metrics i) } \\
\hline & & Total Cites & $\begin{array}{l}\text { Impact } \\
\text { Factor }\end{array}$ & $\begin{array}{l}\text { 5-Year } \\
\text { Impact } \\
\text { Factor }\end{array}$ & $\begin{array}{c}\text { Immediacy } \\
\text { Index }\end{array}$ & Articles & $\begin{array}{c}\text { Cited } \\
\text { Half-life }\end{array}$ & $\begin{array}{c}\text { Eigenfactor }{ }^{\mathrm{TM}} \\
\text { Score }\end{array}$ & $\begin{array}{l}\text { Article Influence } \\
\text { Score }\end{array}$ \\
\hline REV INT METOD NUMER & 0213-1315 & 46 & 0.310 & & 0.000 & 15 & & 0.00014 & \\
\hline
\end{tabular}

Universidad Politécnica de Cataluña

Edificio C-1, Campus Norte-UPC, Gran Capitán S/N

Barcelona 08034, España

- Base de datos: 2009 JCR Science Edition - 


\title{
Cálculo directo de la carga crítica de pandeo de pórticos. Parte I
}

\author{
Antolín Lorenzana Ibán y Mariano Cacho Pérez \\ Aula UVa-CIMNE, ETS de Ingenieros Industriales \\ Universidad de Valladolid \\ Paseo del Cauce, s/n, 47011, Valladolid, España \\ Tel.: 34983 423529, 423391; Fax: 34983423631 \\ e-mail: ali@eis.uva.es; cacho@eis.uva.es
}

\section{Resumen}

De todas las condiciones de diseño de una estructura de barras, en muchos casos, la condición más crítica consiste en asegurar que bajo cualquier combinación posible de cargas no se produzca inestabilidad por flexión, más cuando la tendencia actual es diseñar estructuras esbeltas con aceros de mayor calidad. Resulta por tanto interesante contar con un método de cálculo que permita determinar de forma sencilla y precisa el máximo nivel de carga admisible, conocido habitualmente como carga crítica de pandeo. Para ello, se plantea el equilibrio de cada barra en su configuración deformada, bajo hipótesis de pequeñas deformaciones y pequeños desplazamientos (Teoría de Primer Orden), resultando un sistema de ecuaciones diferenciales lineal para cada barra. Para obtener la respuesta no lineal del conjunto es necesario imponer en cada unión compatibilidad de desplazamientos y equilibrio en el nudo, nuevamente en la configuración deformada. El objetivo de este trabajo es desarrollar un método sistemático que permita determinar la carga crítica y el modo de pandeo de cualquier pórtico plano sin necesidad de recurrir a las simplificaciones que usualmente se asumen en planteamientos matriciales o de elementos finitos. Esto permitirá obtener resultados precisos con independencia de la discretización realizada.

Palabras clave: carga crítica y modo de pandeo, no-linealidad geométrica, Teoría de Primer Orden.

\section{STRAIGHT METHOD FOR CRITICAL BUCKLING LOAD OF FRAMES. PART I}

\begin{abstract}
Summary
Of all the design conditions for frames, in many cases the most critical one consists on ensuring that, under any possible combination of loads, flexural buckling should not take place, specially when the current trend is to design slender structures with high strength steels. Therefore, it is important to have a method to determine in a simple and clear way the maximum acceptable load level, usually known as the critical buckling load. With this purpose, we consider the equilibrium equations of each beam in its deformed configuration, under the hypothesis of infinitesimal strains and displacements (First-Order Theory), resulting in a system of linear differential equations for each element. To obtain the nonlinear response of the frame, it is necessary to impose in each beam-end the compatibility of displacements and the equilibrium also in the deformed configuration. The objective of this work is to develop a systematic method to determine the critical buckling load and the buckling mode of any frame, without using the common simplifications usually assumed in matrix analysis or finite element approaches. This allow to obtain precise results regardless of the discretization done.
\end{abstract}

Keywords: critical buckling load, buckling mode, geometrical non-linearity, First-Order Theory. 


\section{INTRODUCCIÓN}

El problema de calcular la carga crítica de pandeo de vigas bajo cargas de compresión fue ya resuelto por Euler en el $1744^{13}$. Sin embargo, menos usual es encontrar, incluso en la actualidad, herramientas sistemáticas de cálculo que permitan determinar de forma suficientemente precisa, sin un gran esfuerzo de cálculo, el menor nivel de carga que puede originar el pandeo por flexión de cualquier estructura de barras.

Los programas comerciales de aplicación del método de los elementos finitos, en general, resuelven el problema de pandeo con una formulación teórica aproximada que da lugar a la modificación de la rigidez de cada barra mediante la denominada matriz de rigidez geométrica $^{2}$. Con este planteamiento se requiere gran número de elementos por barra para conseguir precisión suficiente de los resultados.

Para evitar estos inconvenientes, en este trabajo se desarrolla una herramienta matemática sencilla, implementada con la ayuda de un manipulador simbólico, que permite calcular de forma precisa la carga crítica de pandeo con deformaciones de flexión de cualquier estructura de barras. Sin pérdida de generalidad se expone el método para el caso plano.

Para alcanzar dicho objetivo, el trabajo se ha organizado de la manera siguiente: en primer lugar, tras esta breve introducción, se presenta el marco teórico para caracterizar el comportamiento no lineal del elemento estructural barra. A continuación se extiende la formulación al análisis de cualquier pórtico plano sometido a cualquier sistema de cargas y condiciones de contorno y se expone cómo determinar para dicho pórtico la carga crítica y el modo de pandeo. En el cuarto apartado se presentan resultados numéricos para tres ejemplos y por último, en base a los resultados se resumen las principales conclusiones obtenidas de este trabajo.

\section{COMPORTAMIENTO DE LA BARRA}

A continuación vamos a obtener el conjunto de ecuaciones que rigen el comportamiento no lineal de una barra aislada. Para ello, en primer lugar, estableceremos las hipótesis básicas adoptadas para seguidamente imponer las condiciones de equilibrio que debe satisfacer cualquier porción de sólido bajo dichas hipótesis dentro del objetivo marcado, junto con las relaciones de compatibilidad-comportamiento.

\section{Hipótesis básicas}

- Se consideran únicamente pórticos planos constituidos por barras esbeltas de directriz recta de sección constante.

- Se supone que los desplazamientos que experimenta la estructura bajo carga son pequeños respecto a la longitud de las barras.

- Se asume que el comportamiento a nivel de punto material, es decir, la relación entre las tensiones y las deformaciones que desarrolla el material que constituye las barras al deformarse, es de tipo elástico-lineal.

- Se consideran cargas aplicadas en los extremos de las barras.

- Se utiliza el modelo de barra de Navier-Bernoulli para barras esbeltas y se desprecia la deformación por cortante.

Comentar que algunas de estas hipótesis, como por ejemplo, sección constante y cargas aplicadas en los nudos, se pueden no exigir y no suponen ninguna modificación del método salvo un mayor esfuerzo computacional. 


\section{Ecuaciones de equilibrio}

En este apartado vamos a establecer las condiciones de equilibrio que debe satisfacer cualquier porción de sólido analizado, más concretamente, cualquier rebanada diferencial de barra.

Antes de pasar directamente a plantear dicho equilibrio, y debido a que en este tipo de análisis el equilibrio debe plantearse en la configuración real, es decir, en la deformada, se hace necesario fijar el sistema de referencia. Vamos a considerar dos sistemas de ejes de referencia a los que referir las magnitudes monodimensionales, desplazamientos y esfuerzos de la barra, que son:

1. Sistema local de la barra $(s, y, z)$ : es un sistema de referencia que varía su orientación con la deformación de tal manera que el eje $s$ siempre es normal a la sección transversal de la barra deformada.

2. Sistema global de la barra $(X, Y, Z)$ : es un sistema de referencia cartesiano de orientación fija independientemente de la deformación de la barra.

En ambos sistemas, tanto el eje $y$ en el local como el eje $Y$ en el global pertenece al plano que contiene la estructura/barra, y por lo tanto, tanto el plano sy como el $X Y$ es el plano en que ésta se deforma.

Por lo tanto, como consecuencia, vamos a disponer de dos conjuntos de ecuaciones de equilibrio, uno por cada sistema de referencia, de idéntico significado físico, pero relativo a referencias distintas.

\section{Sistema de referencia local}

Lógicamente, si empleamos el sistema de referencia local, las magnitudes monodimensionales del modelo de barra, es decir, los desplazamientos y los esfuerzos tendrán la dirección de dichos ejes $(s, y, z)$.

Este hecho da lugar a la definición de esfuerzos que comúnmente se denomina esfuerzos verdaderos o esfuerzos de Cauchy ${ }^{7}$. Seguidamente se impone el equilibrio de cualquier rebanada diferencial de barra según dicha referencia. Para ello hacemos uso de la Figura 1, resultando de dicho equilibrio de fuerzas y momentos, el siguiente sistema de ecuaciones

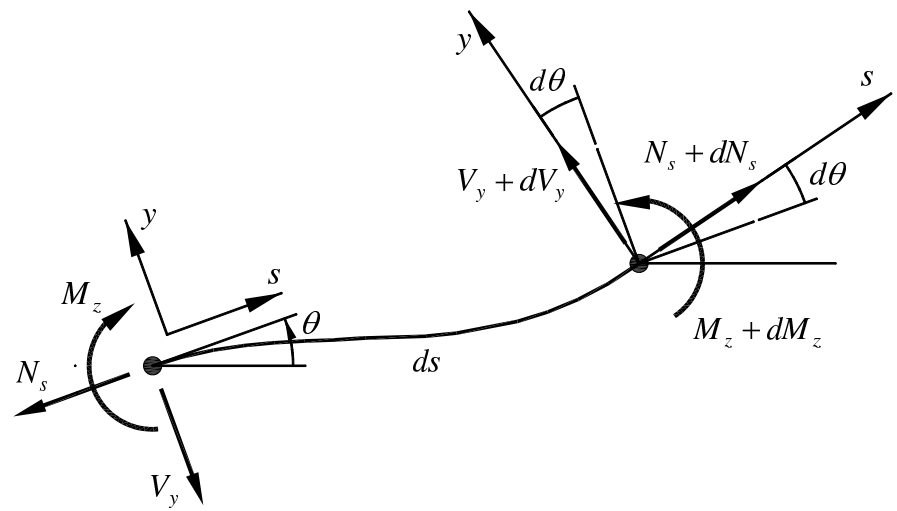

Figura 1. Equilibrio. Sistema local 
diferenciales:

$$
\left\{\begin{array}{c}
N_{s}^{\prime}(s)=V_{y}(s) \theta^{\prime}(s) \\
V_{y}^{\prime}(s)=-N_{s}(s) \theta^{\prime}(s) \\
M_{z}^{\prime}(s)+V_{y}(s)=0
\end{array}\right.
$$

donde las variables primadas indican derivada respecto de la coordenada $s$ donde el ángulo $(\theta)$, es el ángulo girado por la sección de la izquierda del elemento diferencial de longitud $d s$, mientras que la sección de la derecha gira $(\theta+d \theta)$.

\section{Sistema de referencia global}

Otra posibilidad consiste en plantear el equilibrio en la configuración deformada, igual que antes, pero ahora empleando magnitudes estáticas monodimensionales referidas al sistema de referencia global de la barra. Del equilibrio de fuerzas según los ejes $(X, Y)$ y de momentos según $Z$, resulta el siguiente sistema de ecuaciones diferenciales:

$$
\left\{\begin{array}{c}
H^{\prime}(s)=0 \\
V^{\prime}(s)=0 \\
M^{\prime}(s)-H(s) \theta(s)+V(s)=0
\end{array}\right.
$$

donde se ha aproximado el seno del ángulo por el ángulo $(\theta)$ y su coseno por la unidad en base a la hipótesis de pequeños desplazamientos.

Los esfuerzos en este sistema de ejes reciben el nombre de pseudo esfuerzos o esfuerzos de Piola-Kirchhoff? .

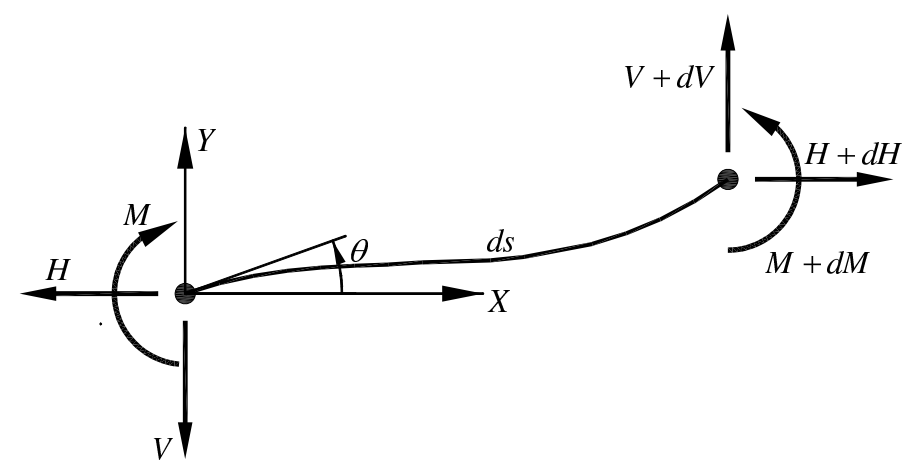

Figura 2. Equilibrio. Sistema global

\section{Ecuaciones de compatibilidad-comportamiento}

Las ecuaciones que relacionan esfuerzos y desplazamientos en base a las hipótesis adoptadas son las usuales de la Resistencia de Materiales ${ }^{3,4}$ :

$$
\left\{\begin{array}{c}
N_{s}(s)=E A u^{\prime}(s) \\
v^{\prime}(s)=\theta(s) \\
M_{z}(s)=E I_{z} \theta^{\prime}(s)
\end{array}\right.
$$




\section{Solución no lineal}

En primer lugar, haremos uso de las expresiones que nos permiten relacionar esfuerzos en ambos sistemas de ejes, local y global, estas son:

$$
\left\{\begin{array}{c}
N_{s}(s)=H(s) \cos (\theta)+V(s) \sin (\theta) \simeq H(s)+V(s) \theta(s) \\
V_{y}(s)=-H(s) \sin (\theta)+V(s) \cos (\theta) \simeq V(s)-H(s) \theta(s) \\
M_{z}(s)=M(s)
\end{array}\right.
$$

El conjunto de ecuaciones anterior (4), las de equilibrio (1) y (2) junto con las ecuaciones de compatibilidad-comportamiento (3), constituyen el siguiente sistema de ecuaciones diferenciales lineales:

$$
\left\{\begin{array}{c}
E A u^{\prime \prime}(s)=V \cdot \theta^{\prime}(s) \\
v^{\prime}(s)=\theta(s) \\
E I_{z} \theta^{\prime \prime \prime}(s)-H \cdot \theta^{\prime}(s)=0
\end{array}\right.
$$

cuya solución general depende del signo de la magnitud $H$, resultando para:

Compresión $(H<0)$ :

$$
\left\{\begin{array}{c}
v(s)=C_{1} \sin (K s)+C_{2} \cos (K s)+C_{3} s+C_{4} \\
\theta(s)=C_{1} K \cos (K s)-C_{2} K \sin (K s)+C_{3} \\
u(s)=C_{5}+C_{6} s-\left(C_{2} C_{3} \cos (K s)+C_{1} C_{3} \sin (K s)\right) \frac{I_{z} K^{2}}{A}
\end{array}\right.
$$

Tracción $(H>0)$ :

$$
\left\{\begin{array}{c}
v(s)=C_{1} \sinh (K s)+C_{2} \cosh (K s)+C_{3} s+C_{4} \\
\theta(s)=C_{1} K \cosh (K s)+C_{2} K \sinh (K s)+C_{3} \\
u(s)=C_{5}+C_{6} s+\left(C_{2} C_{3} \cosh (K s)+C_{1} C_{3} \sinh (K s)\right) \frac{I_{z} K^{2}}{A}
\end{array}\right.
$$

siendo $K=\sqrt{\frac{-H}{E I_{z}}}$ en el primer caso y $K=\sqrt{\frac{H}{E I_{z}}}$ en el segundo .

Conocida la respuesta de cada barra será necesario solamente exigir compatibilidad y equilibrio entre las barras que constituyen la estructura para determinar la respuesta del conjunto.

\section{ANÁLISIS DEL FENÓMENO DE PANDEO}

\section{Equilibrio y compatibilidad en los nudos}

Lo primero de todo, siempre que analizamos estructuras compuestas por más de una barra, es establecer un sistema de referencia común a todas las barras de la estructura, que denominaremos sistema de referencia/ejes global $\left(X_{g}, Y_{g}, Z_{g}\right)$.

El segundo paso es discretizar la estructura/pórtico, es decir, dividir la estructura en un número adecuado de barras. Debido a la formulación empleada en este trabajo y en base a las hipótesis asumidas es suficiente con emplear un número de barras mínimo, coincidente con los tramos rectos entre nudos.

A continuación, una vez identificados los desplazamientos y los esfuerzos de cada barra, en el sistema local o global de barra, debemos pasar dichas magnitudes monodimensionales al sistema de referencia común de toda la estructura. Este cambio de base se realiza en función de la orientación inicial de las barras, dada por el ángulo $\alpha$ de cada barra. 


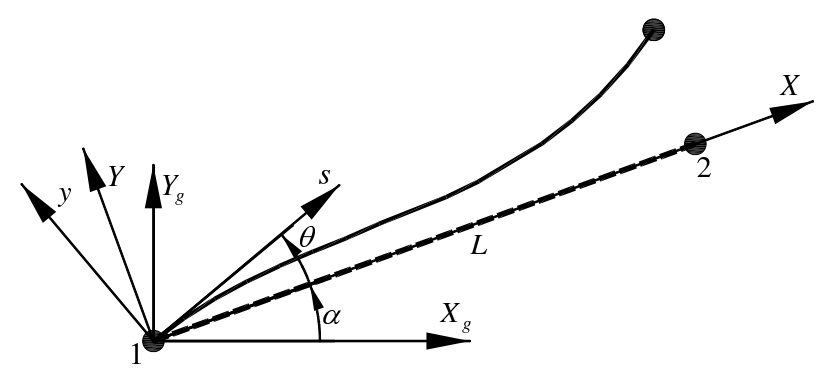

Figura 3. Orientación incial de la barra

Solo queda 'unir' todas las barras para formar el pórtico, este proceso se lleva a cabo imponiendo en cada uno de los nudos de la estructura las correspondientes condiciones de compatibilidad de desplazamientos y de equilibrio de fuerzas y momentos.

En la herramienta de análisis desarrollada, este proceso, aunque sencillo es de casuística muy variada dependiendo de las libertades consideradas en cada nudo, se ha conseguido sistematizar para que una vez definidos los datos del problema (geometría, perfiles, materiales, cargas, apoyos, libertades, etc.) se realice de forma automática sin la intervención del usuario, consiguiendo de esta manera el objetivo buscado.

\section{Condiciones de contorno}

A la vista del orden del sistema de ecuaciones diferenciales ((6) y (7)), es necesario imponer seis condiciones de contorno por barra en desplazamientos y/o esfuerzos para determinar las constantes $C_{i}$. Como es sabido del teorema de unicidad ${ }^{12}$, en cada sección donde se impongan condiciones de contorno, si es conocido el desplazamiento en una determinada dirección el esfuerzo en esa misma dirección será una incógnita del problema y viceversa.

Para un problema plano, toda la casuística posible relativa a la imposición de condiciones de contorno se puede resumir como sigue, por ejemplo, en el extremo $(s=L)$, relativas al sistema de referencia local de la barra, se pueden expresar como:

$$
\left\{\begin{array}{c}
N_{s}(L)=-k_{u} u(L) \\
V_{y}(L)=-k_{v} v(L) \\
M_{z}(L)=-k_{\theta} \theta(L)
\end{array}\right.
$$

Esta representación de las condiciones de contorno, incluye entre otras, las más habituales: apoyo rígido $\left(k_{i}=\infty\right)$, extremo libre $\left(k_{i}=0\right)$, apoyos semirrígidos $\left(0<k_{i}<\infty\right)$, etc.

El siguiente paso será resolver el conjunto de ecuaciones ((6) y (7)) de todas las barras de la estructura junto con las condiciones de contorno correspondiente para determinar, como es usual, el valor de $\lambda$ por el que hay que ponderar el estado de cargas actuante para que aparezcan desplazamientos indeterminados. Se asume por tanto la hipótesis de proporcionalidad de cargas.

\section{Carga crítica de pandeo}

Las ecuaciones de compatibilidad y equilibrio de los nudos, junto con las condiciones de contorno constituyen un sistema de ecuaciones algebraicas de $(3 n) \times(3 n)$ (siendo $n$, es el número de nudos de la estructura) del tipo:

$$
f_{j}\left(C_{i j}, \lambda\right)=F_{j}(\lambda) ; i=1 \ldots 6 ; j=1 \ldots b
$$

siendo $b$ el número de elementos tipo barra con los que se discretiza la estructura. 


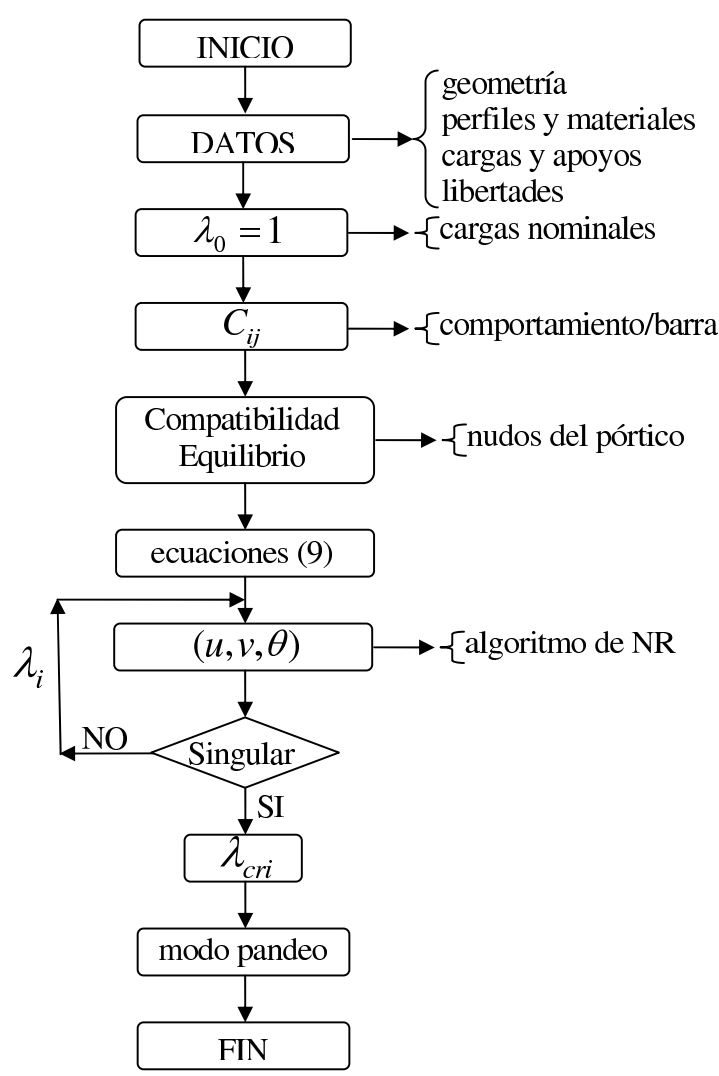

Figura 4. Proceso numérico de cálculo

Para calcular numéricamente la carga crítica de pandeo $\left(\lambda_{c r i}\right)$ será necesario determinar el menor valor del factor $\lambda$ que anula el determinante del sistema de ecuaciones anterior. Dada la no linealidad del sistema (9) se usará un proceso iterativo basado en la técnica numérica de Newton-Raphson. El esquema de resolución se representa en el diagrama de la Figura 4.

\section{Modo de pandeo}

La deformada del pórtico justo en el instante en el que la carga alcanza el valor crítico de pandeo resulta indeterminada, de hecho es lo que se ha buscado al exigir que el determinante del sistema de ecuaciones sea nulo.

Para determinar el modo de pandeo asociado, lo que se hace es resolver el sistema de ecuaciones dado por (9) para el valor de $\lambda_{\text {cri }}$ obtenido, sustituyendo una de las ecuaciones de equilibrio por una condición adicional en desplazamientos, es decir, imponiendo un valor arbitrario para uno de los grados de libertad del pórtico objeto de análisis.

\section{RESULTADOS NUMÉRICOS}

Se presentan tres ejemplos, de complejidad creciente, con los que se pretende mostrar la metodología de cálculo expuesta en los puntos anteriores. 


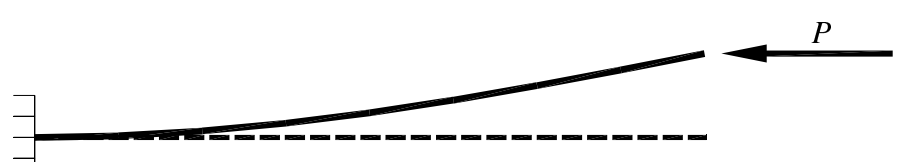

Figura 5. Barra a compresión (modo de pandeo)

\section{Barra a compresión}

Es conocido que la solución exacta al problema de una barra empotrada-libre sometida a compresión es:

$$
P_{c r i}=\frac{\pi^{2} E I_{z}}{(2 L)^{2}}
$$

En este caso sencillo, la estrategia de resolución se reduce a imponer las condiciones de contorno, en desplazamientos en la sección empotrada $(s=0)$ mediante las condiciones $(u(0)=v(0)=\theta(0)=0)$ y en esfuerzos en el extremo libre $(s=L)$ mediante $(V(L)=$ $M(L)=0, H(L)=-P$ ), con lo que el determinante del sistema de ecuaciones dado por (9) tiene como menor raíz:

$$
P_{\text {cri }}=\frac{9,8696 E I_{z}}{(2 L)^{2}}
$$

Se ha resuelto empleando un único elemento y para una tolerancia del proceso de Newton-Raphson (NR) de $10^{-6}$ han sido necesarias solo ocho iteraciones. Los coeficientes $C_{i}$ obtenidos son:

$$
C_{2}=C_{3}=C_{5}=0 ; C_{4}=-C_{1} ; C_{6}=-0,00260289 \cdot C_{1}
$$

El modo de pandeo se muestra con trazo grueso en la Figura 5.

Como inconveniente, debemos comentar que el método iterativo no asegura que la raíz encontrada sea la menor, ya que depende como es lógico de la estimación inicial o punto de partida del algoritmo. Para ello se pueden usar aproximaciones obtenidas mediante otros planteamientos más sencillos, por ejemplo, los basados en la matriz $K_{g}$ de rigidez geométrica ${ }^{2}$, o bien emplear en las primeras iteraciones otras técnicas numéricas como el algoritmo de la Secante y acelerar posteriormente la convergencia mediante el algoritmo de Newton-Raphson.

En este ejemplo concreto, está claro que los resultados son los mismos con independencia de que se considere o no la deformación axil.

Se compara seguidamente la técnica empleada en este trabajo con los resultados obtenidos mediante métodos matriciales basados en $K_{g}$, donde como es sabido la precisión de los resultados depende de la discretización. La Tabla I muestra los resultados para 1, 2, 4, 8 y 16 elementos:

\begin{tabular}{|c|c|c|c|c|c|}
\hline $\mathrm{b}$ & 1 & 2 & 4 & 8 & 16 \\
\hline \hline$P_{c r i}\left(\cdot \frac{E I_{z}}{(2 L)^{2}}\right)$ & 9.94385 & 9.87466 & 9.86993 & 9.86963 & 9.86961 \\
\hline$\varepsilon_{r}(\%)$ & 0.7522 & 0.05122 & 0.003344 & 0.003299 & 0.00005673 \\
\hline
\end{tabular}

Tabla I. Barra a compresión. Método matricial aproximado 


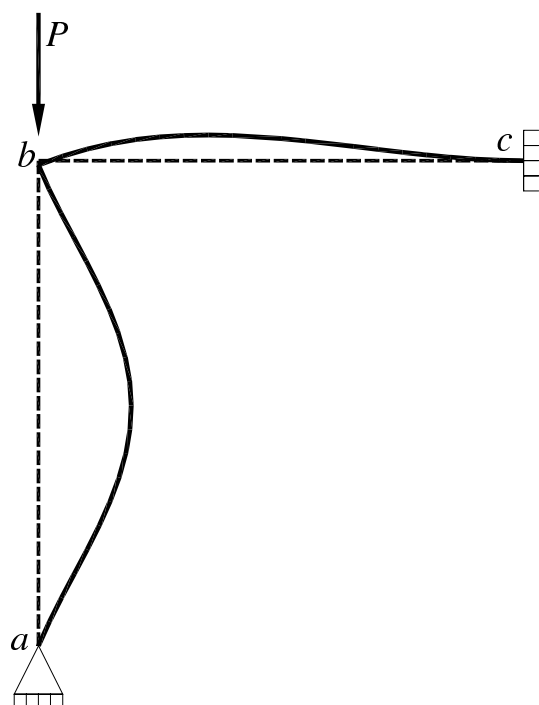

Figura 6. Pórtico de Lee (modo de pandeo)

\section{Pórtico de Lee}

La estructura de la figura, denominada en la literatura 'Pórtico de Lee', permite ilustrar de forma clara y sencilla el objetivo de este trabajo y las posibilidades de generalización de la técnica numérica empleada.

Vamos a considerar por simplicidad que el pórtico está formado por barras iguales en cuanto a longitud $(L)$, material $(E)$ y perfil tubular de diámetro $(D)$ y espesor $(t)$.

Para el caso de la Figura 6, se supone unión rígida en la sección $b$, por lo que las condiciones de compatibilidad y equilibrio adecuadas son:

$$
\left\{\begin{array} { c } 
{ u _ { 1 } ( L ) = v _ { 2 } ( 0 ) } \\
{ v _ { 1 } ( L ) = - u _ { 2 } ( 0 ) } \\
{ \theta _ { 1 } ( L ) = \theta _ { 2 } ( 0 ) }
\end{array} \quad \left\{\begin{array}{c}
-P=H_{1}+V_{2} \\
F=H_{2}-V_{1} \\
M=M_{1}(L)+M_{2}(0)
\end{array}\right.\right.
$$

Tras imponer las condiciones de contorno en los apoyos (secciones a y $c$ ) se llega a un sistema de doce ecuaciones con doce incógnitas, cuya primera raíz real positiva de su determinante es:

$$
P_{c r i}=14,6257 \frac{E I_{z}}{L^{2}}
$$

resultado obtenido tras diez iteraciones para la convergencia con una tolerancia de $10^{-6}$ El modo de pandeo correspondiente se representa con trazo grueso en la propia Figura 6.

Nótese que se podría haber estimado el valor inicial de la carga crítica en el intervalo $\pi^{2} \ldots(\pi / 0,7)^{2}$, que son los valores correspondientes a la carga de pandeo de un pilar biapoyado (caso en el que el dintel $b c$ fuese muy flexible) y otro empotrado-apoyado (caso en el que el dintel fuese muy rígido). También se podría haber tomado como valor inicial de entrada del algoritmo de resolución el obtenido mediante planteamientos matriciales en base a la aproximación de la matriz de rigidez geométrica. En este caso, con el ánimo de comparar 
el esfuerzo computacional, se presentan en la siguiente tabla los resultados para 1, 2, 4, 8 y 16 elementos por barra.

\begin{tabular}{|c|c|c|c|c|c|}
\hline $\mathrm{b} /$ barra & 1 & 2 & 4 & 8 & 16 \\
\hline \hline$P_{c r i}\left(\cdot \frac{E I_{z}}{L^{2}}\right)$ & 20.0847 & 14.8928 & 14.7449 & 14.7328 & 14.732 \\
\hline$\varepsilon_{r}(\%)$ & 37.32 & 1.826 & 0.8153 & 0.7325 & 0.7269 \\
\hline
\end{tabular}

Tabla II. Pórtico de Lee. Método matricial aproximado

Como es sabido, ciertos planteamientos ${ }^{2}$ desprecian de partida la deformación asociada al esfuerzo axil durante el pandeo. Para comprobar la influencia en este caso se presentan los resultados obtenidos con la técnica propuesta para áreas 10, 100 y 1000 veces superior a la inicial y se confirma que hasta cierto punto la hipótesis (no asumida en el planteamiento propuesto) es admisible.

\begin{tabular}{|c|c|c|c|c|}
\hline Área & $A$ & $10 \cdot A$ & $100 \cdot A$ & $1000 \cdot A$ \\
\hline \hline$P_{c r i}\left(\cdot \frac{E I_{z}}{L^{2}}\right)$ & 14.6257 & 14.6567 & 14.6598 & 14.6601 \\
\hline
\end{tabular}

Tabla III. Pórtico de Lee. Influencia de la deformación axil

Como muestra de la versatilidad del método se plantea también la posibilidad de cambiar las condiciones de contorno o de incluir libertades en las uniones entre barras. Así por ejemplo, en el caso de apoyar el pilar mediante un empotramiento la carga crítica aumenta hasta:

$$
P_{c r i}=26,9535 \frac{E I_{z}}{L^{2}}
$$

Y si por ejemplo la unión de ambas barras en $b$ fuera mediante una articulación disminuiría al valor:

$$
P_{c r i}=9,90084 \frac{E I_{z}}{L^{2}}
$$

En este último caso, las condiciones de compatibilidad en el nudo b deben ser:

$$
\left\{\begin{array} { c } 
{ u _ { 1 } ( L ) = v _ { 2 } ( 0 ) } \\
{ v _ { 1 } ( L ) = - u _ { 2 } ( 0 ) }
\end{array} \left\{\begin{array}{c}
-P=H_{1}+V_{2} \\
0=H_{2}-V_{1} \\
0=M_{1}(L) \\
0=M_{2}(0)
\end{array}\right.\right.
$$

\section{Pórtico simple a dos aguas}

Se aplica finalmente la técnica de análisis al pórtico simple a dos aguas indicado en la Figura 7.

Considerando una discretización de sólo cuatro elementos coincidentes con las barras que forman el pórtico y asumiendo un estado proporcional de cargas (siendo $\lambda$ dicho factor de proporcionalidad), resulta, tras un total de siete iteraciones un valor crítico de:

$$
\lambda_{c r i}=3,08654 \cdot 10^{6}
$$




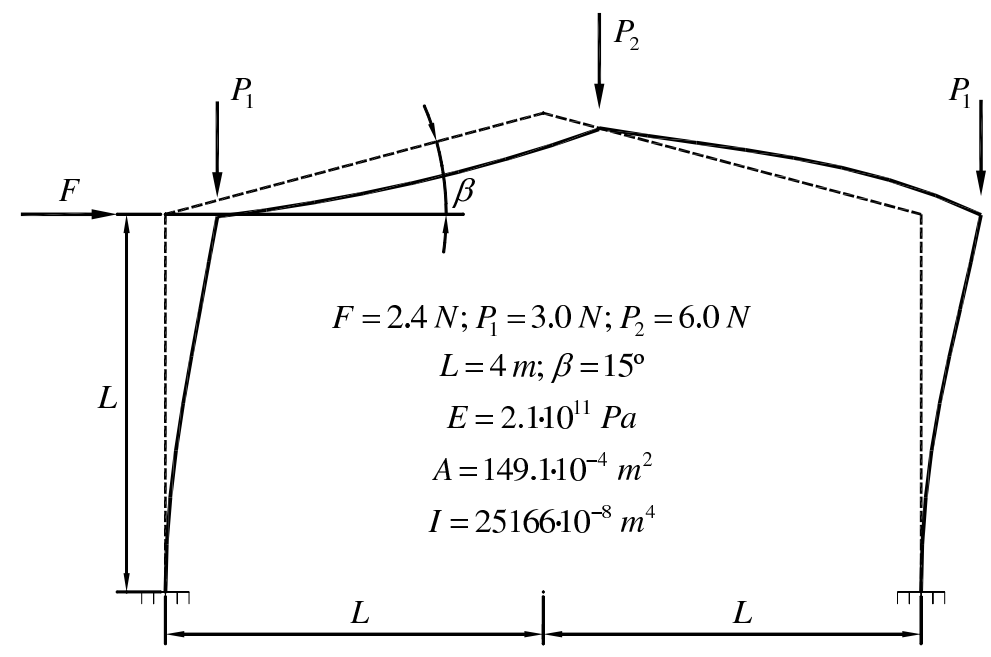

Figura 7. Pórtico simple a dos aguas

Para conseguir resultados con un nivel de precisión similar mediante la técnica de la matriz de rigidez geométrica sería necesario un mínimo de veinte elementos por barra. En la propia Figura 7 se representa el modo de pandeo correspondiente.

\section{CONCLUSIONES}

Para finalizar, vamos a comentar de forma breve la principales conclusiones que se desprenden de este trabajo. En primer lugar, respecto al modelo teórico, el punto de partida ha sido la teoría de pandeo de Euler, que asume pequeños desplazamientos y pequeñas deformaciones, y que da lugar a los conceptos de carga crítica y modo de pandeo, consecuencia del modelo matemático planteado. Por tanto no es posible conocer los desplazamientos en el instante de pandeo ni el posible comportamiento post-pandeo.

En segundo lugar, relativo a la herramienta de análisis desarrollada comentar que es totalmente sistemática, sencilla, y permite incluir modelos más generales, por ejemplo, barras de sección variable, cargas distribuidas sobre las barras, estructuras espaciales (3D), etc. A pesar de que la aplicación informática se ha desarrollado mediante manipulador simbólico (Mathematica v6) no es posible en general obtener soluciones analíticas en función de todos los parámetros, sino sólo numéricas.

Respecto a los resultados numéricos, comprobamos que gracias a una formulación matemática rigurosa (la solución de desplazamientos se basa en funciones de estabilidad), se obtiene la ventaja de que luego, al calcular, se obtienen resultados precisos con discretizaciones mínimas y que para discretizaciones más finas los resultados son prácticamente los mismos.

\section{REFERENCIAS}

1 R.L. Burden y J.D. Faires, Análisis numérico, Thomson, Mexico, (1998).

2 A. Chajes, Principles of Structural Stability Theory, Prentice Hall , (1974).

3 J.A. Garrido y A. Foces, Resistencia de Materiales, Universidad de Valladolid, (2002).

4 J.M. Gere, Timoshenko. Resistencia de Materiales, Thomson, (2005). 
5 M.R. Horne y W. Merchant, The Stability of Frames, Pergamon Press, (1965).

6 C. Lázaro, Formulación material y espacial del modelo geométricamente exacto de piezas alargadas, Universidad Politécnica de Valencia, (2005).

7 S. Monleón, Análisis de vigas, arcos, placas y láminas, una presentación unificada, Universidad Politécnica de Valencia, (1999).

8 M.A. Ortega, J. L. Romero y E. Rosa, Un estudio histórico del problema de las piezas prismáticas rectas sometidas a compresión. Parte I, Informes de la Construcción, Vol. 59, ํㅜ 507, pp. 69-81, Jul-Sept,(2007).

9 M.A. Ortega, J.L. Romero y E. Rosa, Un estudio histórico del problema de las piezas prismáticas rectas sometidas a compresión. Parte II, Informes de la Construcción, Vol. 59, N 508, pp. 61-71, Oct-Dic, (2007).

10 G.J. Simitses y D.H. Hodges, Fundamentals of Structural Stability, Elsevier Inc., (2006).

11 S.P. Timoshenko, Theory of Elastic Stability, McGraw-Hill, (1963).

12 S.P. Timoshenko y J. N. Goodier, Teoría de la Elasticidad, Urmo, (1975).

13 C. Truesdell, Ensayos de Historia de la Mecánica, Tecnos, Madrid, España, (1975). 


\section{b) CÁlCULO DIRECTO DE LA CARGA CRÍTICA} DE PANDEO DE PÓRTICOS. PARTE II

M. Cacho y A. Lorenzana

Rev. Int. Mét. Num. Cálc. Dise. Ing., 26: 31-38, 2010.

\section{Revista Internacional de Métodos Numéricos para Cálculo y Diseño en Ingeniería}

Engineering, Multidisciplinary, Mathematics, Interdisciplinary Applications

\begin{tabular}{|c|c|c|c|c|c|c|c|c|c|}
\hline \multirow[b]{2}{*}{$\begin{array}{l}\text { Abbreviated Journal Title } \\
\text { (linked to journal information) }\end{array}$} & \multirow[b]{2}{*}{ ISSN } & \multicolumn{6}{|c|}{ JCR Data i) } & \multicolumn{2}{|c|}{ Eigenfactor $^{\mathrm{TM}}$ Metrics i) } \\
\hline & & Total Cites & $\begin{array}{l}\text { Impact } \\
\text { Factor }\end{array}$ & $\begin{array}{l}\text { 5-Year } \\
\text { Impact } \\
\text { Factor }\end{array}$ & $\begin{array}{c}\text { Immediacy } \\
\text { Index }\end{array}$ & Articles & $\begin{array}{c}\text { Cited } \\
\text { Half-life }\end{array}$ & $\begin{array}{c}\text { Eigenfactor }{ }^{\mathrm{TM}} \\
\text { Score }\end{array}$ & $\begin{array}{l}\text { Article Influence } \\
\text { Score }\end{array}$ \\
\hline REV INT METOD NUMER & 0213-1315 & 46 & 0.310 & & 0.000 & 15 & & 0.00014 & \\
\hline
\end{tabular}

Universidad Politécnica de Cataluña

Edificio C-1, Campus Norte-UPC, Gran Capitán S/N

Barcelona 08034, España

- Base de datos: 2009 JCR Science Edition - 


\title{
Cálculo directo de la carga crítica de pandeo de pórticos. Parte II
}

\author{
Mariano Cacho Pérez • Antolín Lorenzana Iván
}

Recibido: Abril 2009, Aceptado: Noviembre 2009

(C)Universitat Politècnica de Catalunya, Barcelona, España 2010

Resumen En un artículo anterior se presentó una herramienta matemática para el cálculo sistemático de la carga crítica y el modo de pandeo de cualquier pórtico plano bajo ciertas hipótesis simplificativas. Este trabajo, extiende dicha formulación para proporcionar mayor generalidad, considerando la posibilidad de analizar barras de sección variable bajo de carga (incluyendo cualquier tipo de carga distribuida y variación lineal de la temperatura en el canto del perfil). Para ello, se plantea el equilibrio de cada barra en su configuración deformada, bajo hipótesis de pequeños desplazamientos y pequeñas deformaciones (Teoría de Primer Orden), resultando un sistema de ecuaciones diferenciales de coeficientes variables para cada barra. Para obtener la respuesta no lineal del conjunto es necesario imponer en cada unión compatibilidad de desplazamientos y equilibrio de fuerzas y momentos, nuevamente en la configuración deformada. La solución buscada se obtiene exigiendo que en el instante de pandeo la variación de la energía potencial total sea nula. El objetivo de este trabajo es desarrollar un método sistemático de análisis que permita determinar la carga crítica y el modo de pandeo de estructuras de barras sin necesidad de recurrir a las simplificaciones que usualmente se asumen en planteamientos matriciales o de elementos finitos. Esto permitirá obtener resultados precisos independientemente de la discretización empleada.

Mariano Cacho Pérez · Antolín Lorenzana Ibán Aula UVa-CIMNE, Escuela de Ingenierías Industriales Universidad de Valladolid

Paseo del Cauce 59, 47011, Valladolid, España

Tel.: 34983 423391, 423529; Fax: 34983423631

e-mail: cacho, ali@eis.uva.es

\section{STRAIGHT METHOD FOR CRITICAL BUCKLING LOAD OF FRAMES. PART II}

Summary In a previous article, we presented a mathematical tool for the systematic calculation of the critical buckling load and the buckling mode of any frame, under some simplificative hypothesis. The present work extends this formulation to provide greater generality, considering the possibility of analyzing variable section beams under any kind of loads (including any distributed load and linear variation of the temperature in the edge of the profile). With this purpose, we consider the equilibrium equations of each beam in its deformed configuration, under the hypothesis of infinitesimal strains and displacements, so called First-Order Theory, resulting in a system of differential equations with variable coefficients for each element. To obtain the nonlinear response of the frame, it is necessary to impose in each beam end the compatibility of displacements and the equilibrium of forces and moments, also in the deformed configuration. The solution is obtained by requiring that the total variation of potential energy is zero at the instant of buckling. The objective of this work is to develop a systematic method to determine the critical buckling load and the buckling mode of any frame, without using the common simplifications usually assumed in matrix analysis or finite element approaches. This allows us to obtain precise results regardless of the discretization done.

\section{Introducción}

Como es sabido, las herramientas numéricas de aplicación del método de los elementos finitos, en general, resuelven el problema de pandeo con una formulación teórica aproximada que da lugar a la modificación de la 
rigidez de cada barra mediante la denominada matriz de rigidez geométrica. Con este planteamiento los resultados dependen en gran medida de la discretización empleada y en general requiere de varios elementos por barra para conseguir precisión suficiente.

Para evitar estos inconvenientes, en este trabajo se desarrolla una herramienta matemática sencilla, implementada con la ayuda de un manipulador simbólico, que permite calcular de forma directa la carga crítica y el modo de pandeo con deformaciones de flexión de cualquier estructura plana de barras.

Para alcanzar dicho objetivo, el trabajo se ha organizado de la manera siguiente: en primer lugar, tras esta breve introducción, se presenta el marco teórico para caracterizar el comportamiento no lineal del elemento estructural barra 2D junto con las correspondientes ecuaciones de estabilidad. A continuación se extiende la formulación al análisis de cualquier pórtico plano sometido a condiciones de contorno cualesquiera, y se expone cómo determinar para dicho pórtico la carga crítica y el modo de pandeo asociado. En el cuarto apartado se presentan resultados numéricos para varios ejemplos y por último, en base a los resultados, se resumen las principales conclusiones obtenidas de este trabajo.

\section{Comportamiento de la barra}

\subsection{Hipótesis básicas}

Se asume la hipótesis de pequeñas deformaciones y pequeños desplazamientos y se considera que el comportamiento del material es de tipo lineal. Así mismo, se adopta un estado proporcional de cargas, de tal manera que todas las acciones sobre la estructura, excepto el peso propio y la temperatura, se incrementan en igual proporción respecto a sus valores nominales.

En base a estas hipótesis se presentan a continuación la ecuaciones de equilibrio, compatibilidad y comportamiento para el elemento tipo barra bajo estudio.

\subsection{Equilibrio no lineal}

Antes de pasar a plantear el equilibrio, y debido a que en este tipo de análisis el equilibrio debe plantearse en la configuración real, es decir, en la deformada, se hace necesario fijar el sistema de referencia al que referir desplazamientos y esfuerzos de la barra. Se considera un sistema de referencia cartesiano de orientación fija para cada barra independiente de su deformación, sistema local de la barra que denotamos $(X, Y, Z)$. Del equilibrio de fuerzas según los ejes $(X, Y)$ y de momentos según $Z$, resulta el siguiente sistema de ecuaciones diferenciales en términos de los pseudo esfuerzos o esfuerzos de PiolaKirchhoff [5] (véase la Figura 1):

$\left\{\begin{array}{c}H^{\prime}(s)+q_{X}(s)=0 \\ V^{\prime}(s)+q_{Y}(s)=0 \\ M^{\prime}(s)-H(s) \theta(s)+V(s)=0\end{array}\right.$

donde las variables primadas indican derivada respecto de la coordenada espacial s, y donde se ha aproximado el seno del ángulo por el ángulo y su coseno por la unidad en base a la hipótesis de pequeños desplazamientos.

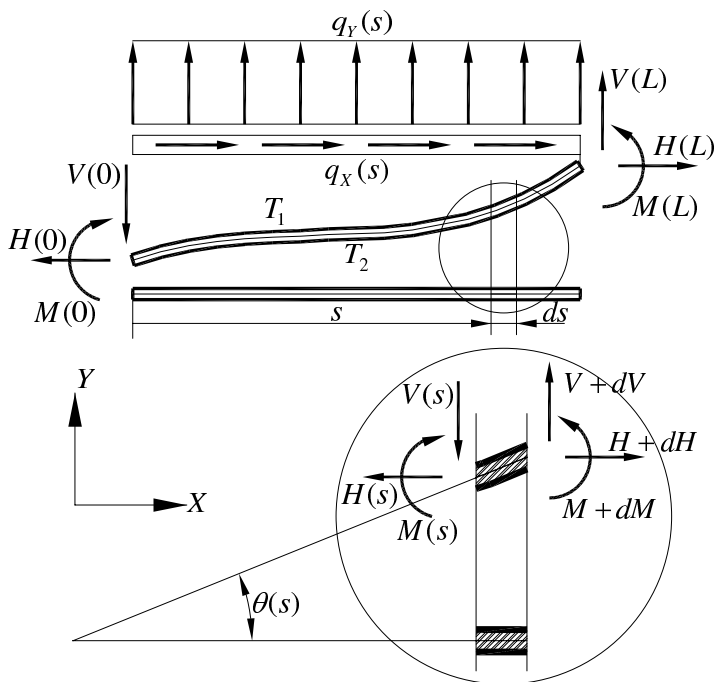

Figura 1. Equilibrio. Sistema local

\subsection{Ecuaciones de compatibilidad-comportamiento}

Las ecuaciones que relacionan los esfuerzos anteriormente mencionados con los desplazamientos $(u, v, \theta)$ de un punto de la directriz de la barra son:

$$
\left\{\begin{array}{c}
H(s)=E A(s)\left(u^{\prime}(s)-\frac{\alpha}{2}\left(T_{1}+T_{2}\right)\right) \\
\theta(s)=v^{\prime}(s) \\
M(s)=E I_{z}(s)\left(\theta^{\prime}(s)+\frac{\alpha}{h(s)}\left(T_{1}-T_{2}\right)\right)
\end{array}\right.
$$

siendo $E$ el módulo de Young del material, $I_{z}(s)$ y $A(s)$ el momento de inercia y el área de cada sección transversal de la barra. Nótese que se incluye el efecto térmico, suponiendo por simplicidad variación lineal de la temperatura en el canto $h(s)$, entre $T_{1} \mathrm{y} T_{2}$, siendo $\alpha$ el coeficiente de dilatación térmica.

Finalmente, las ecuaciones anteriores, las de equilibrio (1) junto con las ecuaciones de compatibilidadcomportamiento (2), constituyen un sistema de ecuaciones diferenciales lineales de coeficientes variables que en 
general no tiene solución matemática cerrada o explícita. No obstante se puede calcular la solución numérica para cada caso concreto analizado mediante distintas técnicas de análisis numérico. Conocida la respuesta de cada barra será necesario solamente exigir compatibilidad y equilibrio entre las barras que constituyen la estructura para determinar la respuesta del conjunto.

\subsection{Ecuaciones de estabilidad}

Como es bien conocido, según aumenta el factor de carga $\lambda$ la respuesta no lineal de cada barra viene dada por la resolución del sistema de ecuaciones diferenciales (1) y (2) con sus correspondientes condiciones de contorno, y dicha solución está en general perfectamente definida (solución estable). Sin embargo, existe un valor del factor de proporcionalidad de carga para el que apararecen repentinamente desplazamientos transversales indeterminados (modo de pandeo).

Para determinar el valor crítico $\lambda_{c r i}$ que puede originar dicho fenómeno, un posible método se basa en introducir una pequeña perturbación del equilibrio que consiste en suponer una deformación infinitesimal adicional de flexión $\Delta u$ respecto al estado estable, lo que da lugar a un nuevo estado de la estructura que lógicamente debe seguir cumpliendo las ecuaciones (1) y (2), de tal manera que las magnitudes incrementales en desplazamientos $(\Delta u, \Delta v, \Delta \theta)$ y en esfuerzos $(\Delta H, \Delta V, \Delta M)$ deben satisfacer las siguientes ecuaciones, que se conocen como ecuaciones de estabilidad [9]:

$$
\begin{gathered}
\left\{\begin{array}{c}
\Delta H^{\prime}(s)=0 \\
\Delta V^{\prime}(s)=0 \\
\Delta M^{\prime}(s)-H(s) \Delta \theta(s)-\theta(s) \Delta H(s)+\Delta V(s)=0
\end{array}\right. \\
\left\{\begin{array}{c}
\Delta H(s)=E A(s) \Delta u^{\prime}(s) \\
\Delta \theta(s)=\Delta v^{\prime}(s) \\
\Delta M(s)=E I_{z}(s) \Delta \theta^{\prime}(s)
\end{array}\right.
\end{gathered}
$$

Estas ecuaciones (3) y (4), junto con las condiciones de contorno asociadas al problema original con solicitaciones exteriores nulas son las que permiten determinar el valor de $\lambda_{c r i}$.

\section{Análisis del fenómeno de pandeo}

\subsection{Equilibrio y compatibilidad en los nudos}

Siempre que se analizan estructuras compuestas por más de una barra, es necesario establecer un sistema de referencia común a todas las barras de la estructura sistema de referencia/ejes global es $\left(X_{g}, Y_{g}, Z_{g}\right)$. Figura 2 .
El segundo paso es discretizar la estructura/pórtico, es decir, dividir la estructura en un número adecuado de barras. Debido a la formulación empleada en este trabajo y en base a las hipótesis asumidas es suficiente con emplear un número de barras mínimo, coincidente con los tramos rectos entre nudos. Discretizaciones más finas llevan a igual solución final a pesar del mayor esfuerzo computacional.

Para pasar las magnitudes monodimensionales de cada barra al sistema de referencia común de toda la estructura, se procede a realizar el correspondiente cambio de base en función de la orientación inicial de las barras, dada por el ángulo $\alpha_{b}$ mediante las expresiones siguientes:

$$
\left[\begin{array}{l}
H \\
V \\
M
\end{array}\right]_{\left(X_{g}, Y_{g}, Z_{g}\right)}=\left[\begin{array}{ccc}
\cos \alpha_{b}-\sin \alpha_{b} & 0 \\
\sin \alpha_{b} & \cos \alpha_{b} & 0 \\
0 & 0 & 1
\end{array}\right] \cdot\left[\begin{array}{c}
H \\
V \\
M
\end{array}\right]_{(X, Y, Z)}
$$

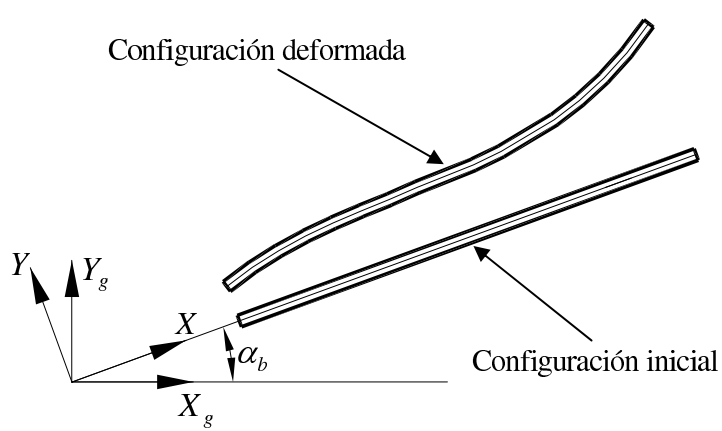

Figura 2. Orientación incial de la barra

Tras estas operaciones sólo resta imponer en cada uno de los nudos de la estructura las correspondientes condiciones de compatibilidad de desplazamientos y de equilibrio de fuerzas y momentos. Este proceso, aunque sencillo, es de casuística muy variada dependiendo de las libertades consideradas en cada nudo. En la aplicación informática desarrollada se ha conseguido sistematizar para que una vez definidos los datos del problema (geometría, perfiles, materiales, cargas, apoyos, libertades, etc.) se realice de forma automática sin la intervención del usuario, consiguiendo de esta manera el objetivo buscado.

\subsection{Condiciones de contorno}

A la vista del orden del sistema de ecuaciones diferenciales (1) y (2), es necesario imponer seis condiciones de contorno por barra en desplazamientos y/o esfuerzos. Como es sabido del teorema de unicidad [12], en cada sección donde se impongan condiciones de contorno, 
si es conocido el desplazamiento en una determinada dirección el esfuerzo en esa misma dirección será una incógnita del problema y viceversa.

Para un problema plano, toda la casuística posible relativa a la imposición de condiciones de contorno se puede resumir como sigue, por ejemplo, en el extremo $(s=L)$, relativas al sistema de referencia global de la barra, se pueden expresar como:

$\left[\begin{array}{l}H(L) \\ V(L) \\ M(L)\end{array}\right]_{\left(X_{g}, Y_{g}, Z_{g}\right)}=-\left[\begin{array}{ccc}k_{u} & 0 & 0 \\ 0 & k_{v} & 0 \\ 0 & 0 & k_{\theta}\end{array}\right] \cdot\left[\begin{array}{l}u(L) \\ v(L) \\ \theta(L)\end{array}\right]_{\left(X_{g}, Y_{g}, Z_{g}\right)}$

Esta representación de las condiciones de contorno, incluye entre otras, las más habituales: apoyo rígido $\left(k_{i}=\infty\right)$, extremo libre $\left(k_{i}=0\right)$, apoyos semirrígidos $\left(0<k_{i}<\infty\right)$, etc.

El siguiente paso será resolver el conjunto de ecuaciones (1) y (2) de todas las barras de la estructura junto con las condiciones de contorno correspondientes para determinar el valor de $\lambda$ por el que hay que ponderar el estado de cargas actuante para que aparezcan desplazamientos de flexión indeterminados.

\subsection{Carga crítica y modo de pandeo}

La deformada del pórtico justo en el instante en el que la carga alcanza el valor crítico de pandeo resulta indeterminada, y por lo tanto, para determinar el modo de pandeo asociado, lo que se hace es resolver las ecuaciones de estabilidad (3) y (4), en función del factor de carga $\lambda$ sustituyendo una de las ecuaciones de equilibrio por una condición adicional en desplazamientos, es decir, imponiendo un valor arbitrario para uno de los grados de libertad del pórtico objeto de análisis.

Para ello, como en las ecuaciones de estabilidad intervienen magnitudes del estado estable o prepandeo, es necesario resolver de forma simultánea las ecuaciones de comportamiento mecánico de cada barra más las ecuaciones de compatibilidad y equilibrio de los nudos, que junto con las condiciones de contorno constituyen un sistema de ecuaciones diferenciales lineal de coeficientes variables cuya solución se puede encontrar mediante técnicas numéricas de resolución de problemas frontera como por ejemplo el algoritmo de disparo no lineal, ya que en la mayoría de los casos no se dispone de una expresión matemática explícita cerrada para dicha solución.

Pues bien, en un artículo anterior, se expuso que para calcular numéricamente la carga crítica de pandeo $\left(\lambda_{c r i}\right)$ era necesario determinar el menor valor del factor $(\lambda)$ que anulaba el determinante del sistema de ecuaciones no lineal resultante. En este trabajo, se emplea un criterio alternativo más general para determinar dicho valor crítico basado en un balance energético. Para ello, se sabe que en el instante de pandeo el pórtico puede deformarse según el modo de pandeo, de tal manera que el valor crítico de carga buscado será aquel que anule el funcional variación de energía potencial total dado por:

$\Delta W(\lambda)=\frac{1}{2} \sum_{i=1}^{b} \int_{0}^{L_{i}}\left(\Delta M_{i}(s) \Delta \theta_{i}^{\prime}(s)+H_{i}(s)\left(\Delta \theta_{i}(s)\right)^{2}\right), d s \quad(7)$

(6) siendo $b$ el número total de barras de la estructura.

Por tanto, la condición $(\Delta W=0)$ permite calcular de forma numérica el valor buscado, mediante el método de la Secante o el algoritmo de Newton-Rapshon.

\section{Resultados numéricos}

Se presentan varios ejemplos, de complejidad creciente, con los que se pretende mostrar la metodología de cálculo expuesta en los puntos anteriores.

\subsection{Barra a compresión (Validación)}

- Caso a

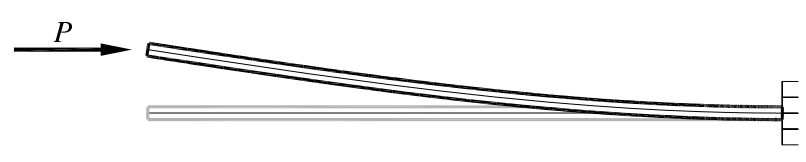

Figura 3. Perfil constante (caso a)

En este caso sencillo, es conocida la solución exacta y corresponde al problema de una barra empotradalibre sometida únicamente a la carga de compresión $P$, ya que aunque pueda existir una variación lineal de la temperatura en el canto, se trata de una estructura isostática. Concretamente en este caso las condiciones de contorno del problema no lineal son:

$H(0)=-P ; V(0)=M(0)=u(L)=v(L)=\theta(L)=0$ de manera que de la integración del sistema de ecuaciones diferenciales constituido por (1) y (2) resulta:

$$
H(s)=-P ; V(s)=M(s)=u(s)=v(s)=\theta(s)=0
$$

Ahora bien, por otro lado, las condiciones de contorno que deben satisfacer las ecuaciones de estabilidad resultan ser:

$\Delta v(0)=v_{0} ; \Delta H(0)=\Delta V(0)=\Delta M(0)=\Delta u(L)=\Delta \theta(L)=0$

donde $v_{0}$ es un valor arbitrario suficientemente pequeño. 
La solución de las ecuaciones de estabilidad (3) y (4) sujetas a dichas condiciones de contorno es:

$$
\left\{\begin{array}{c}
\Delta H(s)=0 \\
\Delta V(s)=\frac{E I_{z} K^{3} v_{0} \cos (K L)}{K L \cos (K L)-\sin (K L)} \\
\Delta M(s)=-\frac{E I_{z} K^{2} v_{0} \sin (K s)}{K L \cos (K L)-\sin (K L)} \\
\Delta u(s)=0 \\
\Delta \theta(s)=\frac{K v_{0}(\cos (K L)-\cos (K s))}{\sin (K L)-K L \cos (K L)} \\
\Delta v(s)=\frac{e^{-i K(L+s)}\left(i e^{i K L}-i e^{i L(L+2 s)}+e^{i K s}(K(L-s)-i)+\right.}{2(K L \cos (K L)-\sin (K L))} \\
\frac{\left.+e^{i K(2 L+s)}(i+K(L-s))\right) v_{0}}{2(K L \cos (K L)-\sin (K L))}
\end{array}\right.
$$

siendo $K=\sqrt{\frac{P}{E I_{z}}}$

El funcional de variación de la energía potencial total se calcula como:

$$
\begin{aligned}
\Delta W(P) & =\frac{1}{2} \int_{0}^{L}\left(\Delta M(s) \Delta \theta^{\prime}(s)-P(\Delta \theta(s))^{2}\right) d s \\
& =\frac{E I_{z} K^{3} v_{0}^{2} \cos (K L)}{\sin (K L)-K L \cos (K L)}
\end{aligned}
$$

y por lo tanto, se comprueba que la carga crítica de pandeo tiene por valor:

$$
P_{c r i}=\frac{\pi^{2} E I_{z}}{(2 L)^{2}}
$$

ya que es el valor que anula el funcional.

- Caso b

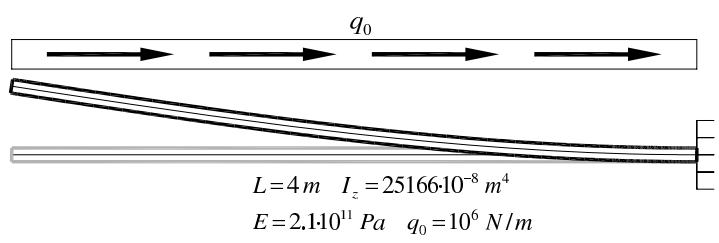

Figura 4. Axil variable (caso b)

En este segundo caso, la barra está sometida a una distribución de carga tal que el esfuerzo axil en la barra no es constante (pilar sometido a peso propio, por ejemplo). Este problema tiene la dificultad de que lógicamente la ecuación diferencial correspondiente resulta de coeficientes variables y aunque puede llevarse a cabo el análisis de inestabilidad mediante un plantemiento matricial y posterior busqueda de raices del determinante de la matriz de rigidez de la estructura, requiere menor esfuerzo computacional el planteamiento energético ya que conlleva el cálculo de una integral. Pues bien, para el caso de axil variable se ha obtenido la siguiente estimación de la carga crítica de pandeo:

\section{$\lambda_{c r i}=6,47176$}

Si comparamos el resultado obtenido con el proporcionado por el paquete de análisis por el método de los elementos finitos Cosmos/M, basado en la linealización del problema no lineal mediante la modificación de la matriz de rigidez de cada barra con su correspondiente matriz de rigidez geométrica, llegamos a la conclusión importante de que lo recomendable es hacer el análisis con un discretización del orden de cincuenta elementos por barra si queremos obtener información suficientemente precisa sobre magnitudes relativas a la estabilidad:

Tabla 1. Barra a compresión. Axil variable. Método matricial

\begin{tabular}{cccccc}
\hline $\mathrm{b}$ & & 10 & 50 & 100 & 200 \\
\hline$\lambda_{\text {cri }}$ & 5.81257 & 6.44510 & 6.47070 & 6.47151 & 6.47171 \\
$\varepsilon_{r}(\%)$ & 10.19 & 0.4119 & 0.01638 & 0.003863 & 0.0007726 \\
\hline
\end{tabular}

- Caso c

Todavía más interesante desde el punto de vista práctico que el caso anterior pueden ser las situaciones en las que el perfil aunque de directriz recta presenta

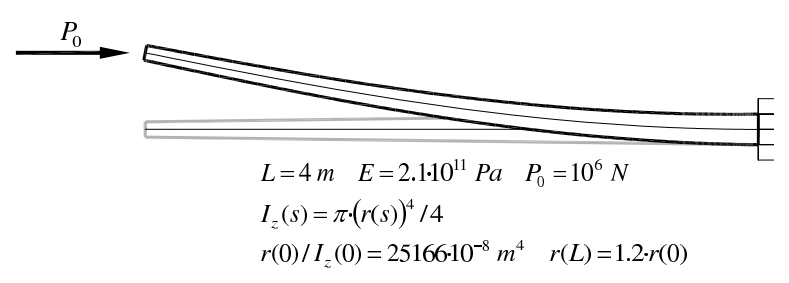

Figura 5. Perfil variable (caso c)

características estáticas (canto, área e inercia) variables en dirección longitudinal. Las ecuaciones diferenciales que rigen el comportamiento mecánico en este caso, aunque lineales son nuevamente de coeficientes variables, de manera que el planteamiento energético presenta clara ventaja operacional frente al matricial, ventaja que aún es mucho mayor cuanto mayor sea el número de barras que forman la estructura. El resultado de la carga crítica obtenido en este caso es:

$\lambda_{\text {cri }}=13,5635$ 
Tabla 2. Barra a compresión. Perfil variable. Método matricial

\begin{tabular}{cccccc}
\hline $\mathrm{b}$ & 2 & 10 & 50 & 100 & 200 \\
\hline$\lambda_{\text {cri }}$ & 10.9825 & 13.0753 & 13.4672 & 13.5154 & 13.5395 \\
$\varepsilon_{r}(\%)$ & 19.03 & 3.599 & 0.7080 & 0.3546 & 0.1769 \\
\hline
\end{tabular}

Como se comprueba, para obtener la misma precisión en los resultados con el planteamiento matricial de la aplicación Cosmos/M es necesario irse a discretizaciones muy finas.

- Caso d

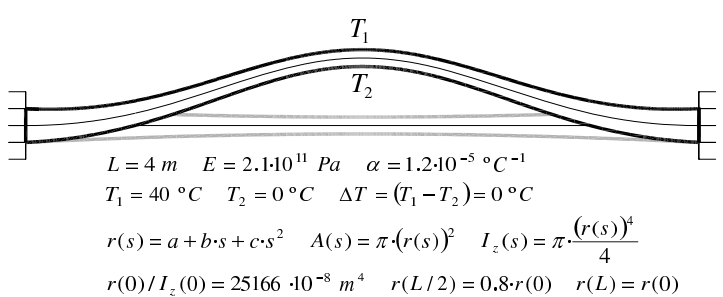

Figura 6. Temperatura (caso d)

Como último ejemplo de validación vamos a considerar la inestabilidad por efecto térmico en una barra biempotrada. En primer lugar, comprobamos que para el caso de perfil de propiedades estáticas constantes el resultado obtenido coincide con el teórico: $\left(r(s) / I_{z}(s)=25166 \cdot 10^{-8} m^{4}\right)$

$N_{c r i}=\frac{(2 \pi)^{2} E I_{z}}{L^{2}}=E A \alpha \lambda_{c r i} \Delta T$

$\lambda_{\text {cri }}=46,0077$

Comparado con Cosmos/M:

Tabla 3. Barra a compresión. Temperatura. Método matricial

\begin{tabular}{cccccc}
\hline $\mathrm{b}$ & 2 & 10 & 50 & 100 & 200 \\
\hline$\lambda_{\text {cri }}$ & 46.0547 & 46.0431 & 46.0272 & 46.0261 & 46.0242 \\
$\varepsilon_{r}(\%)$ & 0.1022 & 0.07694 & 0.04238 & 0.03999 & 0.03586 \\
\hline
\end{tabular}

Para el caso de perfil variable (variación longitudinal del canto de tipo cuadrático) la solución es:

$\lambda_{\text {cri }}=35,9735$

En la siguiente tabla se compara con la solución obtenida con la aplicación Cosmos/M:
Tabla 4. Barra a compresión. Temperatura y perfil variable. Método matricial

\begin{tabular}{cccccc}
\hline $\mathrm{b}$ & 2 & 10 & 50 & 100 & 200 \\
\hline$\lambda_{c r i}$ & 38.5335 & 36.0012 & 35.9883 & 35.9881 & 35.9881 \\
$\varepsilon_{r}(\%)$ & 7.116 & 0.077 & 0.04114 & 0.04059 & 0.04059 \\
\hline
\end{tabular}

\subsection{Pórtico de Lee (Aplicación 1)}

La estructura de la figura, denominada en la literatura [3] 'Pórtico de Lee', permite ilustrar de forma clara y sencilla el objetivo de este trabajo y las posibilidades de generalización de la técnica numérica empleada.

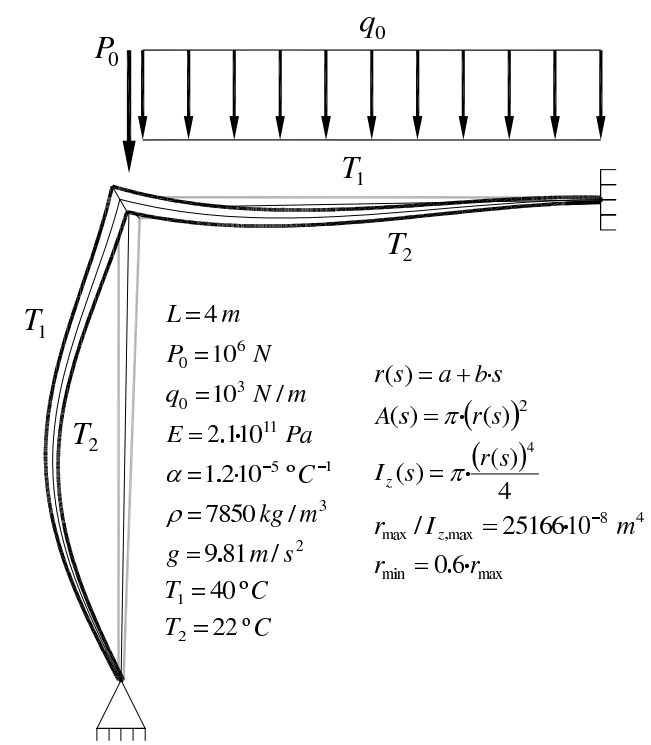

Figura 7. Pórtico de Lee. Perfiles de inercia variable

Inicialmente se considera por simplicidad que el pórtico está formado por barras iguales en cuanto a longitud $(L)$, material $(E)$ y perfil $\left(I_{z}\right)$, y se supone unión rígida en la sección $b$. Llevando a cabo un balance energético entre el instante de prepandeo y el de pandeo, y para los valores numéricos indicados, se llega al siguiente valor del factor de carga crítico que origina el pandeo por flexión en el plano del pórtico:

$$
\lambda_{\text {cri }}=47,5063
$$

El modo de pandeo asociado correspondiente a cada caso se indica a trazo grueso sobre cada una de las figuras.

Lo más interesante del planteamiento de este trabajo, consiste en que permite dotar al análisis de una 


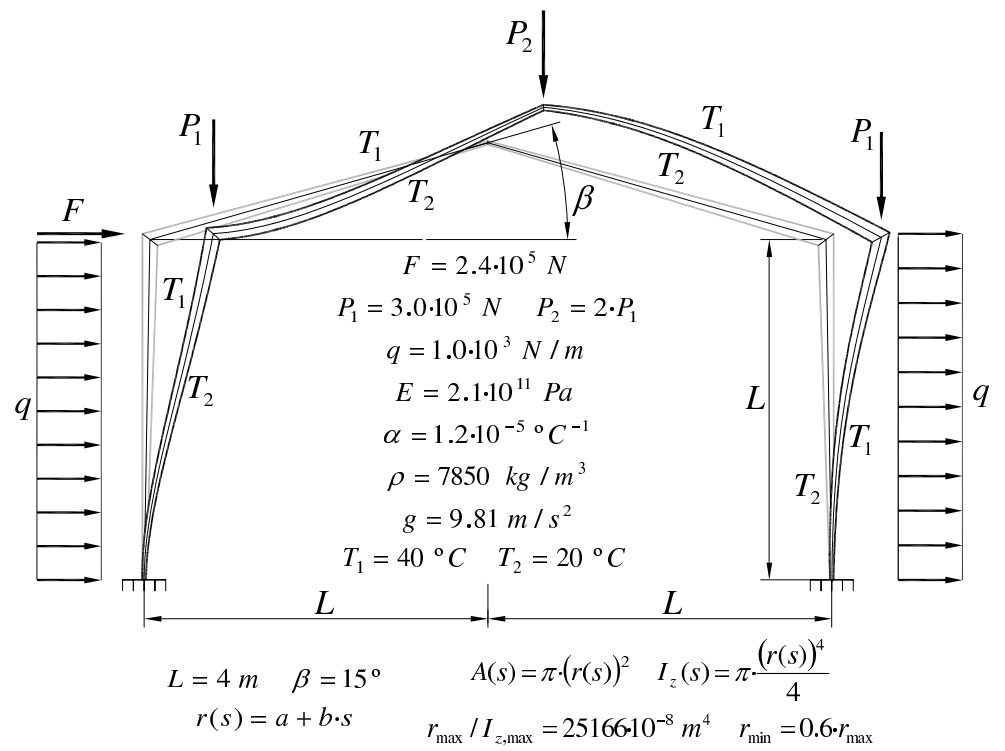

Figura 8. Pórtico simple a dos aguas. Perfiles de inercia variable.

gran generalidad, y así, se puede considerar cualquier tipo de carga, el efecto del peso propio, efectos de origen térmico e incluso variación del perfil en direccion longitudinal de las barras. Concretamente a continuación, se estudia el fenómeno de pandeo en el pórtico de Lee de la Figura 7.

Para el caso de propiedades estáticas variables resulta un factor de carga crítico de valor:

$\lambda_{c r i}=14,5092$

\subsection{Pórtico simple a dos aguas (Aplicación 2)}

Se aplica finalmente la técnica de análisis al pórtico simple a dos aguas indicado en la Figura 8:

Considerando una discretización de sólo cuatro elementos coincidentes con las barras que forman el pórtico y asumiendo un estado proporcional de cargas (siendo $\lambda$ dicho factor de proporcionalidad), resulta para el caso de perfil de inercia constante $\left(r(s) / I_{z}(s)=25166\right.$. $10^{-8} m^{4}$ ), un valor crítico:

$\lambda_{\text {cri }}=29,1906$

Mientras que para el caso recogido en la Figura 8, perfil variable en dirección longitudinal de barra, el factor de carga crítico es bastante menor, concretamente:

$\lambda_{\text {cri }}=9,39849$
Para conseguir resultados con un nivel de precisión similar mediante la técnica de la matriz de rigidez geométrica sería necesario un mínimo de cincuenta elementos por barra.

\section{Conclusiones}

Para finalizar, se comentan de forma breve las principales conclusiones que se desprenden de este trabajo. En primer lugar, respecto al modelo teórico, el punto de partida ha sido la teoría de pandeo de Euler, que asume pequeños desplazamientos y pequeñas deformaciones, y que da lugar a los conceptos de carga crítica y modo de pandeo, consecuencia del modelo matemático planteado. Por tanto no es posible conocer los desplazamientos en el instante de pandeo ni el posible comportamiento post-pandeo.

En segundo lugar, relativo a la herramienta de análisis desarrollada comentar que es totalmente sistemática, sencilla, y permite incluir modelos generales, por ejemplo, barras de sección variable, cargas distribuidas sobre las barras, estructuras espaciales (3D), etc. A pesar de que la aplicación informática se ha desarrollado mediante manipulador simbólico (Mathematica v7) no es posible en general obtener soluciones analíticas en función de todos los parámetros, sino sólo numéricas.

Respecto a los resultados numéricos, se comprueba que gracias a una formulación matemática rigurosa, se obtiene la ventaja de que luego, al calcular, se obtienen resultados precisos con discretizaciones mínimas y que para discretizaciones más finas los resultados son prácticamente los mismos. 


\section{Referencias}

1. Truesdell C. (1975) Ensayos de Historia de la Mecánica. Tecnos. Madrid. España

2. Burden R.L., Faires J.D. (1998) Análisis numérico. Thomson. Mexico

3. Ortega M.A., Romero J.L., Rosa E. (2007) Un estudio histórico del problema de las piezas prismáticas rectas sometidas a compresión. Parte I. Informes de la Construcción. 59 (507):69-81. Jul-Sept

4. Ortega M.A., Romero J.L., Rosa E. (2007) Un estudio histórico del problema de las piezas prismáticas rectas sometidas a compresión. Parte II. IInformes de la Construcción. 59(508):61-71. Oct-Dic

5. Monleón S.(1999) Análisis de vigas, arcos, placas y láminas, una presentación unificada. Universidad Politécnica de Valencia
6. Lázaro C. (2005) Formulación material y espacial del modelo geométricamente exacto de piezas alargadas. Universidad Politécnica de Valencia

7. Garrido J.A., Foces A. (2002) Resistencia de Materiales. Universidad de Valladolid

8. Gere J.M. (2005) Timoshenko. Resistencia de Materiales. Thomson

9. Simitses G.J., Hodges D.H. (2006) Fundamentals of Structural Stability. Elsevier Inc.

10. Timoshenko S.P. (1963) Theory of Elastic Stability. McGraw-Hill

11. Horne M.R., Merchant W. (1965) The Stability of Frames. Pergamon Press

12. Timoshenko S., Goodier J.N. (1975) Teoría de la Elasticidad. Urmo 


\section{c) OPTIMIZACIÓN GLOBAL DE PÓRTICOS 2D CON BARRAS DE SECCIÓN VARIABLE}

M. Cacho Pérez y A. Lorenzana Ibán

DYNA Ingeniería e Industria.

Aceptado: junio 2010.

Publicación, fecha prevista: noviembre 2010.

\section{DYNA Ingeniería e Industria}

Engineering, Multidisciplinary

\begin{tabular}{|c|c|c|c|c|c|c|c|c|c|}
\hline \multirow[b]{2}{*}{$\begin{array}{l}\text { Abbreviated Journal Title } \\
\text { (linked to journal information) }\end{array}$} & \multirow[b]{2}{*}{ ISSN } & \multicolumn{6}{|c|}{ JCR Data i) } & \multicolumn{2}{|c|}{ Eigenfactor ${ }^{\mathrm{TM}}$ Metrics $\mathrm{i}$} \\
\hline & & Total Cites & $\begin{array}{l}\text { Impact } \\
\text { Factor }\end{array}$ & $\begin{array}{l}\text { 5-Year } \\
\text { Impact } \\
\text { Factor }\end{array}$ & $\begin{array}{l}\text { Immediacy } \\
\text { Index }\end{array}$ & Articles & $\begin{array}{l}\text { Cited } \\
\text { Half-life }\end{array}$ & $\begin{array}{c}\text { Eigenfactor }^{\mathrm{TM}} \\
\text { Score }\end{array}$ & $\begin{array}{l}\text { Article Influence } \\
\text { Score }\end{array}$ \\
\hline DYNA-BILBAO & $0012-7361$ & 26 & 0.062 & & 0.000 & 62 & & 0.00002 & \\
\hline
\end{tabular}

Federación Asociaciones Ingenieros Industriales

Alameda de Mazarredo 69

Bilbao 48009, España

- Base de datos: 2009 JCR Science Edition - 


\title{
OPTIMIZACIÓN GLOBAL DE PÓRTICOS 2D CON BARRAS DE SECCIÓN VARIABLE
}

\author{
Mariano Cacho Pérez ${ }^{1}$ y Antolín Lorenzana Ibán ${ }^{2}$ \\ Grupo de Mecánica de Solidos y Estructuras. \\ Escuela de Ingenierías Industriales. Universidad de Valladolid (UVa).
}

Paseo del Cauce 59, 47011, Valladolid (España)

\begin{abstract}
Resumen: El cálculo y dimensionado de estructuras fue una de las primeras disciplinas en demandar potentes herramientas de cálculo. Los métodos para realizar las necesarias comprobaciones sobre resistencia, estabilidad, vibraciones, etc. son muy exigentes desde el punto de vista computacional y usualmente asumen restrictivas simplificaciones como perfiles de sección constante, linealización de problemas no lineales, etc. Sin embargo, con las capacidades actuales tanto de cálculo como de fabricación y el uso de nuevos materiales, junto con ciertos condicionantes estéticos, es posible abordar problemas como el que se presenta en este artículo, donde se busca la variación óptima del canto de las barras de cualquier pórtico de manera que se cumplan cuantos criterios sean exigibles, entre ellos el de estabilidad, es decir, que no aparezcan fenómenos de pandeo. Para barras aisladas existen soluciones clásicas, en algunos casos analíticas, para la forma que debe tener una barra comprimida de manera que su resistencia al pandeo sea máxima. Pero para estructuras de barras el problema es más complejo y debe resolverse de forma numérica. En este trabajo se presenta una formulación novedosa para resolver el problema de optimización de pórticos teniendo en cuenta los condicionantes no solo de pandeo sino cualquier otro, como por ejemplo tensiones admisibles, desplazamientos limitados, etc . Se seleccionan ciertos parámetros de diseño y se formula matemáticamente el problema de optimización para determinar qué valores maximizan la carga de pandeo del pórtico sujeto a las restricciones de diseño (material, tensiones, desplazamientos, etc.). Para ello, se plantea el equilibrio de cada barra en su configuración deformada, bajo hipótesis de pequeños desplazamientos y pequeñas deformaciones (Teoría de Segundo Orden), dando lugar a un sistema de ecuaciones diferenciales de coeficientes variables que se resuelve numéricamente mediante programación cuadrática secuencial.
\end{abstract}

Palabras clave: optimización, carga crítica y modo de pandeo, inercia variable.

\section{GLOBAL OPTIMIZATION OF 2D FRAMES WITH VARIABLE CROSS-SECTION BEAMS}

\begin{abstract}
:
The structural design has been one of the first fields of engineering in needing powerful tools for analysis. The methods to asses that the structure agrees with any of the design criteria (strength, stability, vibrations,...) are usually implemented in numerical applications that, even under usual simplifications such as constant cross-section or linearization, are computationally very demanding. Nevertheless, with the current capacities both of analysis and of manufacturing and the use of new materials, it is possible to propose problems like the ones shown in this work, where the variation of the dimensions of the cross-section of the beams of any 2D frame is determined so that its strength to buckle is maximum. Classic solutions exist for isolated beams, in some cases even analytical solutions. But for structures built of several beams, the problem is more complex and numerical solutions are compulsory. The new formulation presented in this work can deal with the optimization problem of frames in which the geometrical shape of the profile is found under restrictions such as stability (no buckling), elastic behavior (equivalent von Mises stress below the yield stress), limited displacements, etc. With these aim, equilibrium equations for each beam are established in its deformed configuration, and using the hypothesis of small displacements and small deformations (Second Order Theory) a system of differential equations of variable coefficients is set. For its solution, numerical techniques as quadratic sequential programming are employed.
\end{abstract}

Keywords: optimization, critical load and buckling mode, variable cross-section

\footnotetext{
${ }^{1}$ Profesor Asociado, Doctorando; e-mail: cacho@eis.uva.es; tel.: +34 983423391

${ }^{2}$ Doctor Ingeniero Industrial, Profesor Titular; e-mail: ali@eis.uva.es; tel.: +34 983423529
} 


\section{1.- INTRODUCCIÓN}

El comportamiento mecánico de elementos comprimidos ha sido una fuente de aportaciones teóricas y de resultados prácticos de gran interés para la ingeniería del que han surgido importantes áreas como la estabilidad estructural. El primer científico que se ocupó de estudiar el comportamiento resistente de un elemento prismático fue Galileo en su publicación de 1638, donde trata de obtener algunos resultados sobre la resistencia de una viga sometida a cargas transversales, este problema, y, en particular, el de determinación de la elástica o deformada de la viga, es conocido como el problema de Galileo.

Respecto al problema de pandeo, J. Benoulli (1705) obtiene de manera precisa la ecuación de la elástica basándose en la hipótesis de Mariotte sobre la fibra neutra. Euler, en 1744, mediante su método de cálculo de variaciones obtiene, a partir de la sugerencia de D. Bernoulli, la ecuación diferencial de la elástica y obtiene también el valor de la carga de pandeo. En 1757, obtiene de nuevo el valor de dicha carga a partir de una simplificación (ecuación linealizada) de la ecuación diferencial de la elástica. En publicaciones posteriores, considera el caso de piezas de sección variable y otros con carga axial distribuida a lo largo de la longitud de la pieza. Al mismo tiempo se profundiza en distintas líneas de trabajo. En 1770, Lagrange[20] estudia la ecuación linealizada de Euler e investiga el valor de las cargas de pandeo superiores a la primera para la pieza biarticulada. Así mismo, se propuso hallar la forma que debería tener una columna de altura y volumen dados para que aguantase la máxima compresión posible sin pandear, y establece erróneamente que la mejor forma es un cilindro circular, en otras palabras, que el éntasis no aumenta la resistencia. Posteriormente, Clausen, en 1851, determina la pieza óptima frente al pandeo, la forma de columna más estable es aquélla donde la variación de la sección circular a lo largo del fuste lo constituye una curva parecida a una cicloide[22]. En relación con este problema, hay multitud de publicaciones de gran interés. Keller[7] y Tadjbakhsh[18], derivaron que las formas geométricas óptimas de la pieza más resistente frente al fenómeno de pandeo es una cuya sección varía de la misma forma que la determinada por Clausen, pero, en lugar de secciones circulares, éstas son triángulos equiláteros que mantienen el paralelismo de los lados y con el baricentro en el eje de la pieza.

El trabajo de Keller ha despertado gran interés en el área. Taylor[19] estudió el mismo problema usando un enfoque energético, y presentó un límite inferior para el máximo autovalor. Spillers y Levy[17] extendieron el problema del pandeo de una columna al del diseño óptimo de una placa frente a inestabilidades por flexión y más tarde al estudio del fenómeno de pérdida de estabilidad de una cubierta cilíndrica simétrica a lo largo de un eje[16]. Sin embargo, un inconveniente con todos estos trabajos es que sus respectivos autores limitaron sus diseños óptimos a sólo una restricción: un volumen constante. En la práctica, sin embargo, las restricciones impuestas por la resistencia del material empleado o por los desplazamientos juegan un papel igualmente importante.

$\mathrm{Fu}$ y Ren[4] retomaron los trabajos antes mencionados, aunque agregando las restricciones de tensiones necesarias, planteando así el problema de minimizar el volumen de una columna sujeta a una cierta carga mediante el ajuste de su forma geométrica. El método que utilizaron para resolver este problema fue el gradiente reducido generalizado, obteniendo resultados muy favorables.

Existen también referencias muy recientes, que emplean la teoría matemática del análisis funcional para obtener la forma óptima frente a pandeo por torsión y/o flexión a partir de las condiciones que establece el principio de Pontriyagin, Atanackovic[1].

Como se ha podido comprobar, el campo de la optimización estructural ha atraído la atención de un gran número de investigadores desde hace tiempo. Hay numerosas publicaciones recientes[3][6][8][11][14] donde se presentan, en un marco multidisciplinar, distintas técnicas numéricas. Sin embargo, en relación al estudio de fenómenos de inestabilidad, los trabajos en general se limitan al análisis de barras y consideran muy pocas restricciones de diseño, la de volumen y en el mejor de los casos se añade la comprobación a resistencia. Por estos motivos, este trabajo se ha centrado en optimizar pórticos planos, con una función objetivo planteada en términos de estabilidad y en cuanto a restricciones o condiciones de diseño se pueden considerar las más habituales: volumen, tensiones y desplazamientos, aunque se puede añadir cualquier otra.

Por último, indicar que el trabajo se ha organizado de la manera siguiente: en primer lugar, tras esta introducción, se presenta el marco teórico para caracterizar el comportamiento no lineal de la barra y calcular la carga crítica y el modo de pandeo. A continuación, se extiende la formulación al análisis de pórticos planos, seguido de una breve descripción del problema y de la estrategia de optimización. En los dos últimos apartados, se presentan los resultados numéricos para varios ejemplos y se resumen las principales conclusiones obtenidas de este trabajo. 


\section{2.- MODELO DE BARRA}

Se parte, como es habitual en Resistencia de Materiales, de las hipótesis básicas de pequeñas deformaciones y pequeños desplazamientos y del modelo de barra de Navier-Bernoulli, con comportamiento del material de tipo elástico lineal. Así mismo, se adopta un estado proporcional de cargas, de tal manera que todas las acciones sobre la estructura, excepto el peso propio y la temperatura, se incrementan en igual proporción respecto a sus valores nominales, mediante el factor de carga $(\lambda)$.

En base a estas hipótesis se presentan a continuación las ecuaciones de equilibrio, compatibilidad y comportamiento para el elemento tipo barra objeto de estudio.

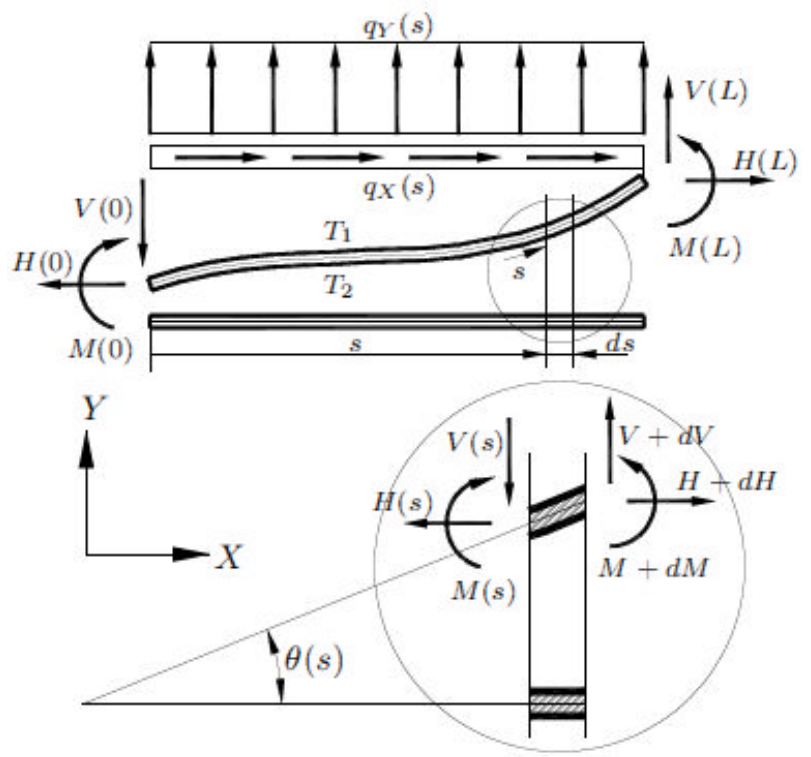

Figura 1.- Equilibrio.

\subsection{Comportamiento no-lineal. Prepandeo.}

Se considera un sistema de referencia cartesiano de orientación fija para cada barra independiente de su deformación, sistema local de la barra denotado por $(X, Y, Z)$. Del equilibrio de fuerzas según los ejes $(X, Y)$ y de momentos según $Z$ en la configuración deformada, resulta el siguiente sistema de ecuaciones diferenciales en términos de los pseudo-esfuerzos o esfuerzos de Piola-Kirchhoff[9]:

$$
\left\{\begin{array}{l}
H^{\prime}(s)+q_{X}(s)=0 \\
V^{\prime}(s)+q_{Y}(s)=0 \\
M^{\prime}(s)-H(s) \theta(s)+V(s)=0
\end{array}\right.
$$

donde las variables primadas indican derivada respecto de la coordenada espacial s, y donde se ha aproximado el seno del ángulo por el ángulo y su coseno por la unidad en base a la hipótesis de pequeños desplazamientos.

Las ecuaciones que relacionan los esfuerzos anteriormente mencionados con los desplazamientos $(u, v, \theta)$ de un punto de la directriz de la barra son:

$$
\left\{\begin{array}{l}
H(s)=E A(s)\left(u^{\prime}(s)-\frac{\alpha}{2}\left(T_{1}+T_{2}\right)\right) \\
\theta(s)=v^{\prime}(s) \\
M(s)=E I_{z}(s)\left(\theta^{\prime}(s)+\frac{\alpha}{h(s)}\left(T_{1}-T_{2}\right)\right)
\end{array}\right.
$$

siendo $E$ el módulo de Young del material, $I_{z}(s)$ y $A(s)$ el momento de inercia y el área de cada sección transversal de la barra. Nótese que se incluye el efecto térmico, suponiendo por simplicidad variación lineal de la temperatura en el canto $h(s)$, entre $T_{1}$ y $T_{2}$, siendo $\alpha$ el coeficiente de dilatación térmica.

Finalmente, las ecuaciones anteriores, las de equilibrio (1) junto con las ecuaciones de compatibilidadcomportamiento (2), constituyen un sistema de ecuaciones diferenciales lineales de coeficientes variables que 
en general no tiene solución matemática cerrada o explícita. No obstante, se puede calcular la solución numérica para cada caso concreto analizado mediante distintas técnicas de análisis numérico. Conocida la respuesta de cada barra será necesario solamente exigir compatibilidad y equilibrio entre las barras que constituyen la estructura para determinar la respuesta del conjunto.

\subsection{Ecuaciones de estabilidad. Pandeo global.}

La solución de las ecuaciones (1) y (2) para unas determinadas condiciones de contorno en general es única. Sin embargo, para el planteamiento de Teoría de Segundo Orden adoptado, ciertos valores discretos de las cargas, denominados cargas críticas, podría no ser así. Significa que puede producirse un respuesta distinta de la esperada (solución estable o estado prepandeo), dando lugar a súbitos desplazamientos de amplitud indeterminada. En esta situación la forma de la deformada se conoce como modo de pandeo.

Para determinar la carga crítica, un posible método[15] se basa en introducir una pequeña perturbación del equilibrio. Consiste en suponer un desplazamiento transversal infinitesimal $\Delta \underline{u}$ respecto al estado estable. En esta nueva situación se deben seguir cumpliendo las ecuaciones (1) y (2), de tal manera que las magnitudes incrementales en desplazamientos $(\Delta u, \Delta v, \Delta \theta)$ y en esfuerzos $(\Delta H, \Delta V, \Delta M)$ deben satisfacer las siguientes ecuaciones lineales, que se conocen como ecuaciones de estabilidad:

$$
\begin{aligned}
& \left\{\begin{array}{l}
\Delta H^{\prime}(s)=0 \\
\Delta V^{\prime}(s)=0 \\
\Delta M^{\prime}(s)-H(s) \Delta \theta(s)-\theta(s) \Delta H(s)+\Delta V(s)=0
\end{array}\right. \\
& \left\{\begin{array}{l}
\Delta H(s)=E A(s) \Delta u^{\prime}(s) \\
\Delta \theta(s)=\Delta v^{\prime}(s) \\
\Delta M(s)=E I_{z}(s) \Delta \theta^{\prime}(s)
\end{array}\right.
\end{aligned}
$$

Estas ecuaciones, junto con las condiciones de contorno asociadas al problema original con cargas exteriores nulas son las que permiten determinar la carga crítica. Desde el punto de vista matemático consiste en obtener los autovalores del problema de frontera definido por dichas ecuaciones (3) y (4).

La deformada del pórtico justo en el instante en el que la carga alcanza el valor crítico resulta indeterminada, y por lo tanto, para calcular el modo de pandeo asociado, lo que se hace es resolver las ecuaciones de estabilidad (3) y (4) sustituyendo una de las ecuaciones de equilibrio por una condición adicional en desplazamientos, es decir, imponiendo un valor arbitrario para uno de los grados de libertad del pórtico objeto de análisis (de ahí la denominación de modo).

Para ello, y debido a que en las ecuaciones de estabilidad intervienen magnitudes del estado estable o prepandeo, es necesario resolver de forma simultánea las ecuaciones de comportamiento mecánico de cada barra más las ecuaciones de compatibilidad y equilibrio de los nudos, que junto con las condiciones de contorno constituyen un sistema de ecuaciones diferenciales lineal de coeficientes variables cuya solución se puede encontrar mediante técnicas adecuadas. Aunque para algunos casos, autores como Keller[7] presenten soluciones analíticas basadas en el cálculo de variaciones, en el caso general son necesarios algoritmos numéricos como el método del disparo no lineal, diferencias finitas o el método de Rayleigh-Ritz[15].

La deformada obtenida de esta manera se puede escalar o normalizar, atendiendo a alguna norma. Se recuerda que para poder conocer la deformada real, y por tanto el comportamiento post-pandeo, es necesario recurrir a modelos matemáticos más complejos, que caen dentro de lo que se conoce como teoría de "grandes desplazamientos".

\section{3.- ANÁLISIS DE PÓRTICOS}

En la sección anterior se ha indicado el conjunto de ecuaciones que describe el comportamiento no-lineal de la barra aislada. En este apartado se describe el método de análisis que permite determinar la respuesta del conjunto de barras que forma el pórtico objeto de estudio en cada caso concreto. Para ello, se necesitan las condiciones de contorno junto con las relaciones de equilibrio de fuerzas y momentos en los nudos de unión de las distintas barras y las condiciones de compatibilidad en desplazamientos y giros.

A diferencia de los métodos clásicos de análisis donde en general se recurre a simplificaciones (planteamiento matricial y linealización) y por ello es necesario emplear un alto número de elementos por barra para poder conseguir la precisión suficiente, en este trabajo se ha empleado un método de análisis directo y no aproximado, de esa manera se consigue una mayor generalidad y un ahorro de cómputo al no requerir mallados finos ni matriz de rigidez alguna. 


\subsection{Equilibrio y Compatibilidad.}

Siempre que se analizan estructuras compuestas por más de una barra, es necesario establecer un sistema de referencia común a todas las barras de la estructura llamado sistema de referencia global (Xg, Yg, Zg). Seguidamente es necesario discretizar la estructura/pórtico, es decir, dividirla en un número adecuado de barras. Debido a la formulación empleada en este trabajo y en base a las hipótesis asumidas es suficiente con emplear un número de barras coincidente con los tramos rectos entre nudos. Discretizaciones más finas llevan a igual solución final a pesar del mayor esfuerzo computacional.

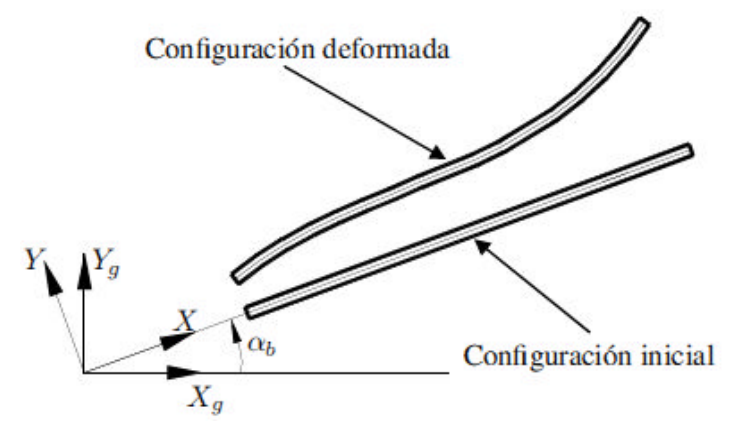

Figura 2.- Orientación inicial de la barra.

Para pasar las magnitudes monodimensionales de cada barra al sistema de referencia común de toda la estructura, se procede a realizar el correspondiente cambio de base en función de la orientación inicial de las barras, dada por el ángulo $\alpha_{b}$ mediante las expresiones siguientes:

$$
\left[\begin{array}{c}
H \\
V \\
M
\end{array}\right]_{\left(X_{q}, Y_{q}, Z_{q}\right)}=\left[\begin{array}{ccc}
\cos \alpha_{b} & -\sin \alpha_{b} & 0 \\
\sin \alpha_{b} & \cos \alpha_{b} & 0 \\
0 & 0 & 1
\end{array}\right] \cdot\left[\begin{array}{l}
H \\
V \\
M
\end{array}\right]_{(X, Y, Z)}
$$

Tras estas operaciones, sólo resta imponer en cada uno de los nudos de la estructura las correspondientes condiciones de compatibilidad de desplazamientos y de equilibrio de fuerzas y momentos. Este proceso, aunque sencillo, es de casuística muy variada dependiendo de las libertades consideradas en cada nudo. En la aplicación informática desarrollada[3][8] en MATLAB ${ }^{\circledR}[2][5]$ se ha conseguido sistematizar para que una vez definidos los datos del problema (geometría, perfiles, materiales, cargas, apoyos, libertades, etc.) se realice de forma automática sin la intervención del usuario.

\subsection{Condiciones de contorno}

A la vista del orden del sistema de ecuaciones diferenciales (1) y (2), es necesario imponer seis condiciones de contorno por barra en desplazamientos y/o esfuerzos. Como es sabido del teorema de unicidad[21], en cada sección donde se impongan condiciones de contorno, si es conocido el desplazamiento en una determinada dirección, el esfuerzo en esa misma dirección será una incógnita del problema y viceversa.

Para un problema plano, toda la casuística posible relativa a la imposición de condiciones de contorno se puede resumir como sigue, por ejemplo, en el extremo $(s=L)$, relativas al sistema de referencia local de la barra, se pueden expresar como:

$$
\left[\begin{array}{c}
H(L) \\
V(L) \\
M(L)
\end{array}\right]_{\left(X_{q}, Y_{q}, Z_{q}\right)}=-\left[\begin{array}{ccc}
k_{u} & 0 & 0 \\
0 & k_{v} & 0 \\
0 & 0 & k_{\theta}
\end{array}\right] \cdot\left[\begin{array}{l}
u(L) \\
v(L) \\
\theta(L)
\end{array}\right]_{\left(X_{q}, Y_{q}, Z_{q}\right)}
$$

Esta representación de las condiciones de contorno, incluye entre otras, las más habituales: apoyo rígido $\left(k_{i}=\infty\right)$, extremo libre $\left(k_{i}=0\right)$, apoyos semirrígidos $\left(0<k_{i}<\infty\right)$, etc.

El siguiente paso será resolver el conjunto de ecuaciones ((1)-(4)) de todas las barras de la estructura junto con las condiciones de contorno correspondientes para determinar el valor de la carga de pandeo. 


\section{4.- OPTIMIZACIÓN}

El esfuerzo en este trabajo se ha enfocado hacia formular un método numérico que permita dimensionar de forma óptima las dimensiones de la sección transversal en ciertas cotas de las barras que conforman un pórtico. Para ello, entre otras posibilidades, se ha planteado una función objetivo en términos del factor de carga crítico, sujeta a restricciones relativas al estado tensional, desplazamientos, cantidad de material utilizado, etc. Esto permite resolver problemas condicionados por fenómenos de inestabilidad, al igual que otros gobernados por el comportamiento a flexión. Tanto la función objetivo, como las restricciones, en general son no lineales, motivo por el que se ha elegido como técnica de optimización un método de Programación No Lineal, concretamente el algoritmo de Programación Cuadrática Sucesiva (SQP, Sequential Quadratic Programming[10][12]) junto con la herramienta de computación técnica MATLABß[2][5]. Además, el código de programación se ha desarrollado de forma que la elección de variables resulte sencilla y versátil, con posibilidad de modificar la función objetivo, y por último, de manera que sea muy simple tanto eliminar como añadir nuevas restricciones al problema.

\subsection{Variables de decisión. Parámetros de diseño.}

Con ánimo de no complicar la exposición del método y buscando una fácil visualización de los resultados se plantea que la sección transversal de las barras dependa de un solo parámetro $r$, que puede ser el radio si la sección es circular, el canto para sección en doble T, etc. Este parámetro puede variar a lo largo de la coordenada espacial $s$ de cada barra $r(s)$. Se supone sin pérdida de generalidad, un tipo de variación polinómica en función de un número finito de parámetros $\left(a_{i}\right)$, si bien es posible plantear cualquier otra función:

$$
r(s)=a_{0}+a_{1} s+a_{2} s^{2}+\ldots+a_{n} s^{n}
$$

Estas simplificaciones permiten ilustrar de forma clara la metodología y comparar algunos resultados con los disponibles en la literatura[1]. Con este procedimiento se valida el método (apartado 5.1) antes de extender su aplicación a casos de mayor interés (ejemplos 5.2 y 5.3). En 5.1 se busca la variación óptima del diámetro de la sección transversal de una columna circular maciza, y en 5.2 y 5.3 el canto en los extremos de los pilares y dinteles de pórticos, donde por razones constructivas se asume que la ley de variación es lineal.

\subsection{Función objetivo}

Con el propósito de poder optimizar incluso perfiles sometidos a compresión centrada, donde el criterio que condiciona el diseño es precisamente la aparición del fenómeno de pandeo con deformaciones de flexión planteado en los apartados previos, se establece como función objetivo:

$$
\max _{a_{i}} \lambda_{c r i}\left(a_{i}\right)
$$

Se trata por tanto de buscar el máximo valor del factor $\lambda_{\text {rri }}$ que provoca el pandeo del conjunto.

\subsection{Restricciones}

La primera restricción que se ha considerado es la de limitar la cantidad de material empleado. Un posible planteamiento consiste en fijar o limitar dicho valor de tal manera que la solución final con barras de sección variable no pese más que la inicial construida con barras de sección uniforme:

$$
\sum_{i=1}^{b} \int_{0}^{L_{i}} \pi r_{i}^{2}(s) d s \leq \sum_{i=1}^{b} \int_{0}^{L_{i}} \pi r_{0, i}^{2} d s=V_{0}
$$

siendo $b$, el número de barras o elementos que forman el pórtico.

La segunda restricción es que la tensión equivalente máxima no supere en ningún punto de la estructura la máxima tensión de trabajo del material, es decir, que sea siempre menor que la tensión de fluencia. Para ello se debe adoptar algún criterio de plastificación, como por ejemplo el de Von-Mises para materiales dúctiles:

$$
\begin{aligned}
& \sigma=\frac{\left|H_{i}(s)\right|}{A_{i}(s)}+\frac{\left|M_{i}(s) \cdot r_{i}(s)\right|}{2 \cdot I_{z, i}(s)} \\
& \tau=\frac{V_{i}(s) \cdot Q_{z, i}(s, y)}{b_{i}(y) \cdot I_{z, i}(s)} \\
& \sigma_{e q v}=\sqrt{\sigma^{2}+3 \cdot \tau^{2}} \leq \sigma_{F}
\end{aligned}
$$


En estas expresiones se consideran las tensiones normales debidas al esfuerzo axil y momento flector, y las tensiones tangenciales debidas al esfuerzo cortante como usualmente se hace en el cálculo de estructuras metálicas. Para estructuras de hormigón armado se hacen necesarias otras comprobaciones, tanto globales como locales, y otros criterios, y en principio no se consideran en este trabajo. De igual modo, y con ánimo de no complicar la exposición del método de optimización, no se han considerado los coeficientes de mayoración de las acciones y/o minoración de las resistencias del material comunmente incluidos en las normativas (CTE, Eurocódigos, etc.), y se ha trabajado por lo tanto únicamente con valores nominales.

Nótese que las restricciones (9) y (10), al igual que la función objetivo (8) son funciones fuertemente no lineales.

Se pueden añadir las condiciones de diseño que resulten necesarias como nuevas restricciones del problema. Por ejemplo, entre otras se puede limitar la respuesta de la estructura en deformaciones y desplazamientos, en frecuencias propias, etc. Incluso cabe la posibilidad de otros planteamientos del problema como puede ser fijar un valor mínimo de la carga crítica (nueva restricción) y buscar la solución geométrica del pórtico en función de los parámetros de diseño escogidos que minimiza la cuantía de material (nueva función objetivo).

\section{5.- RESULTADOS NUMÉRICOS}

Se presentan a continuación varios ejemplos de complejidad creciente, con los que se pretende mostrar la metodología de cálculo expuesta en los puntos anteriores, los valores numéricos comunes para todos los ejemplos son:

$$
\begin{array}{lll}
E=2.1 \cdot 10^{5} \mathrm{MPa} & \rho=7850 \frac{\mathrm{kg}}{\mathrm{m}^{3}} & \alpha=1.2 \cdot 10^{-5} \frac{\mathrm{m}}{\mathrm{m}^{\circ} \mathrm{C}} \\
\sigma_{F}=275 \mathrm{MPa} ; & T_{1}=40^{\circ} \mathrm{C} & T_{2}=20^{\circ} \mathrm{C}
\end{array}
$$

\subsection{Viga empotrada-libre}

En este ejemplo, a modo de validación, se resuelve el problema de una barra (de sección circular maciza) empotrada-libre sometida a carga de compresión $(P)$, carga distribuida transversal $(q)$, peso propio $(\rho)$ y una variación lineal de la temperatura en el canto $\left(T_{1}, T_{2}\right)$ mostrado en Figura 3. La geometría óptima corresponde a un fuste con una variación del radio según una curva tipo cicloide tal y como demostraron los trabajos de Clausen, Keller[7] y Tadjbakhsh[18], y que queda claramente justificado mediante la teoría matemática de análisis funcional en el reciente trabajo de Atanackovic[1]. La carga crítica (función objetivo a maximizar) se obtiene en cada iteración del proceso de optimización como el menor de los valores propios del problema de frontera correspondiente, que en este caso concreto queda descrito por el siguiente sistema de ecuaciones diferenciales:

$$
\begin{aligned}
& \left\{\begin{array}{l}
M^{\prime \prime}(s)+\lambda P \theta(s)+\lambda q+\rho g A(s)=0 \\
v^{\prime}(s)=\theta(s) \\
M(s)=E I_{z}(s)\left(\theta^{\prime}(s)+\frac{\alpha}{h(s)}\left(T_{1}-T_{2}\right)\right)
\end{array}\right. \\
& \text { s.a. }:\left\{\begin{array}{l}
v(0)=0, \theta(0)=0 \\
V(L)=0, M(L)=0
\end{array}\right.
\end{aligned}
$$

Como referencia con la que comparar los resultados obtenidos en los casos de inercia variable se incluyen los resultados correspondientes al caso de sección uniforme de radio $r(s)=r_{0}=12 \mathrm{~cm}$, para el caso numérico: $L=4 m, P_{1}=10^{5} \mathrm{~N}$ y $q=10^{4} \frac{\mathrm{N}}{\mathrm{m}}$.

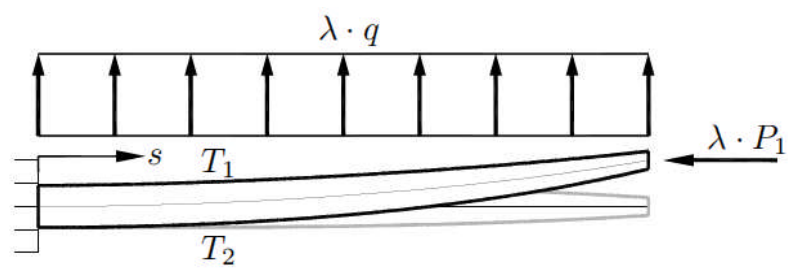

Figura 3.- Viga empotrada-libre, modo de pandeo. 
Buscando la forma óptima, se presentan resultados para los casos de variación lineal, cuadrática y cúbica del canto a lo largo de la longitud de la viga. Los valores óptimos calculados se muestran en la Tabla 1 y se dibujan en la Figura 4. Se comprueba algo que es bien sabido, que la carga transversal $(q)$, el peso propio $(\rho)$ y la variación térmica $\left(T_{1}, T_{2}\right)$ no influyen en el valor de la carga crítica, aunque lógicamente si que afecta a la respuesta en tensiones y/o desplazamientos de la viga.

\begin{tabular}{|c|c|c|l|c|}
\cline { 2 - 5 } \multicolumn{1}{c|}{} & uniforme & \multicolumn{1}{c|}{ lineal } & \multicolumn{1}{c|}{ cuadrática } & \multicolumn{1}{c|}{ cúbica } \\
\cline { 2 - 5 } & $r(s)=r_{0}$ & $r(0)=1.25142 \cdot r_{0}$ & $r(0)=1.10858 \cdot r_{0}$ & $r(0)=1.13642 \cdot r_{0}$ \\
\cline { 3 - 5 } & & $r(L)=0.725333 \cdot r_{0}$ & $r(L / 2)=1.065 \cdot r_{0}$ & $r(L / 3)=1.124 \cdot r_{0}$ \\
\cline { 3 - 5 } & & & $r(L)=0.531 \cdot r_{0}$ & $r(2 L / 3)=0.956833 \cdot r_{0}$ \\
\cline { 3 - 5 } & & & & $r(L)=0.480083 \cdot r_{0}$ \\
\hline$\lambda_{\text {cri }}$ & 52.7418 & 65.623 & 69.309 & 69.741 \\
\hline
\end{tabular}

Tabla 1.- Viga empotrada-libre, resultados.

De los resultados se puede concluir que la solución para variaciones polinómicas de mayor grado se aproxima a la solución teórica presentada por Keller[7], si bien en dicha publicación no se ha considerado la restricción en tensiones, de ahí que en el extremo libre el canto no sea nulo como ocurre en [7].

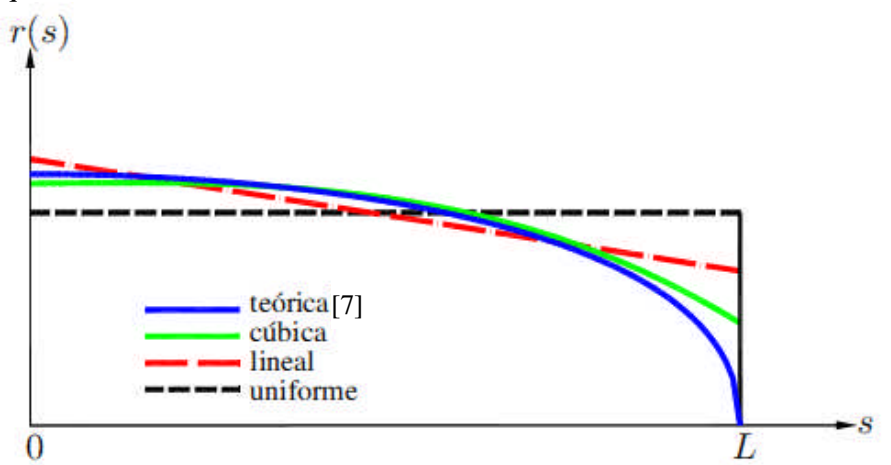

Figura 4.- Viga empotrada-libre, variación longitudinal del canto (escala: vertical (10:1), horizontal (1:1)).

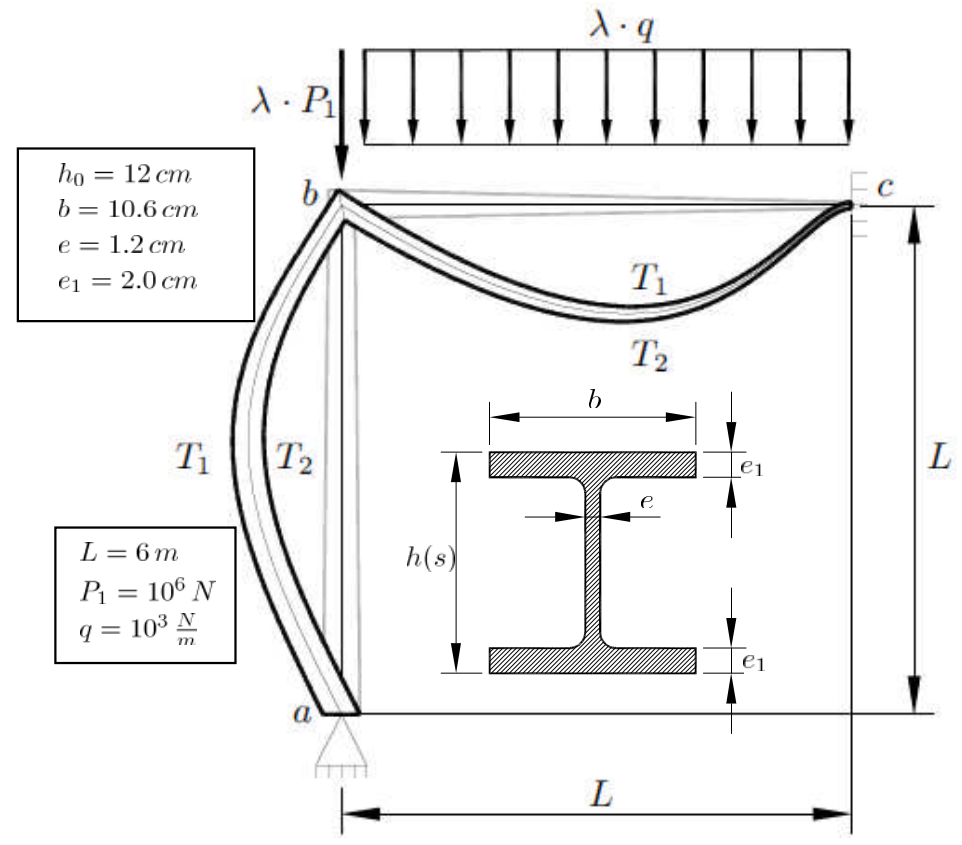

Figura 5.- Pórtico de Lee, modo de pandeo. 


\subsection{Pórtico de Lee}

La estructura denominada en la literatura 'Pórtico de Lee'[13], permite ilustrar de forma clara y sencilla el objetivo de este trabajo y las posibilidades de generalización de la técnica numérica empleada.

Se considera inicialmente que el pórtico está formado por barras iguales en cuanto a longitud $(L)$, material $(E, \rho, \alpha)$ y perfil (sección tipo doble T, correspondiente a un HEM100: $\left.h_{0}, b, e, e_{1}\right)$, y se supone unión rígida pilar-dintel. Desde el punto de vista práctico, puede resultar muy interesante diseñar el pórtico con perfiles de inercia variable. Para una variación lineal del canto cabe preguntarse cómo dimensionar dichos perfiles para que la estructura tenga máxima resistencia al pandeo. Para ello se han considerado tres parámetros (valor del canto en las secciones $-a, b, c-$ ), y tras el proceso numérico se obtienen los resultados indicados en la Tabla 2 para las condiciones de contorno mostradas en la Figura 5.

\begin{tabular}{|ll|ll|}
\hline \multicolumn{2}{|l|}{ perfil uniforme } & \multicolumn{2}{|c|}{ variación lineal del canto } \\
$r_{a}=h_{0}$ & $\lambda_{c r i}=0.957$ & $r_{a}=20.841 \mathrm{~cm}$ & $\lambda_{c r i}=1.568$ \\
$r_{b}=h_{0}$ & $\sigma_{e q v}=217.3864 \mathrm{MPa}$ & $r_{b}=9.893 \mathrm{~cm}$ & $\sigma_{e q v}=213.2783 \mathrm{MPa}$ \\
$r_{c}=h_{0}$ & $r_{c}=7.373 \mathrm{~cm}$ & \\
\hline
\end{tabular}

Tabla 2.- Pórtico de Lee, resultados.

Como muestra de las posibilidades del método se puede cambiar de manera simple las condiciones de contorno y del tipo de unión entre barras. Sse contempla la posibilidad de considerar cualquier tipo de libertad interna entre cada una de las barras que concurran en un mismo nudo, lo que permite dotar al método de análisis de una gran versatilidad. Así, por ejemplo, suponiendo empotrada la base del pilar (sección $a$ ) y unión articulada pilar-dintel (sección $b$ ) la solución hubiera sido:

\begin{tabular}{|ll|ll|}
\hline perfil uniforme & variación lineal del canto \\
$r_{a}=h_{0}$ & $\lambda_{c r i}=1.323$ & $r_{a}=23.370 \mathrm{~cm}$ & $\lambda_{\text {cri }}=2.333$ \\
$r_{b}=h_{0}$ & $\sigma_{\text {eqv }}=192.7055 \mathrm{MPa}$ & $r_{b}=9.310 \mathrm{~cm}$ & $\sigma_{\text {eqv }}=205.4909 \mathrm{MPa}$ \\
$r_{c}=h_{0}$ & $r_{c}=6.010 \mathrm{~cm}$ & \\
\hline
\end{tabular}

Tabla 3.- Pórtico de Lee, base del pilar empotrado y unión articulada pilar-ditel.

\subsection{Pórtico simple a dos aguas}

Se aplica finalmente la técnica de análisis a un pórtico simple a dos aguas, sometido a las cargas mostradas en la Figura 6 y suponiendo que todos los nudos son rígidos. Se buscará la solución simétrica de mayor resistencia al pandeo suponiendo, como en el ejemplo anterior, la posibilidad de variación lineal del canto. En estas condiciones la solución para $r$ en las secciones -a,b,c- se muestra en la Tabla 4.

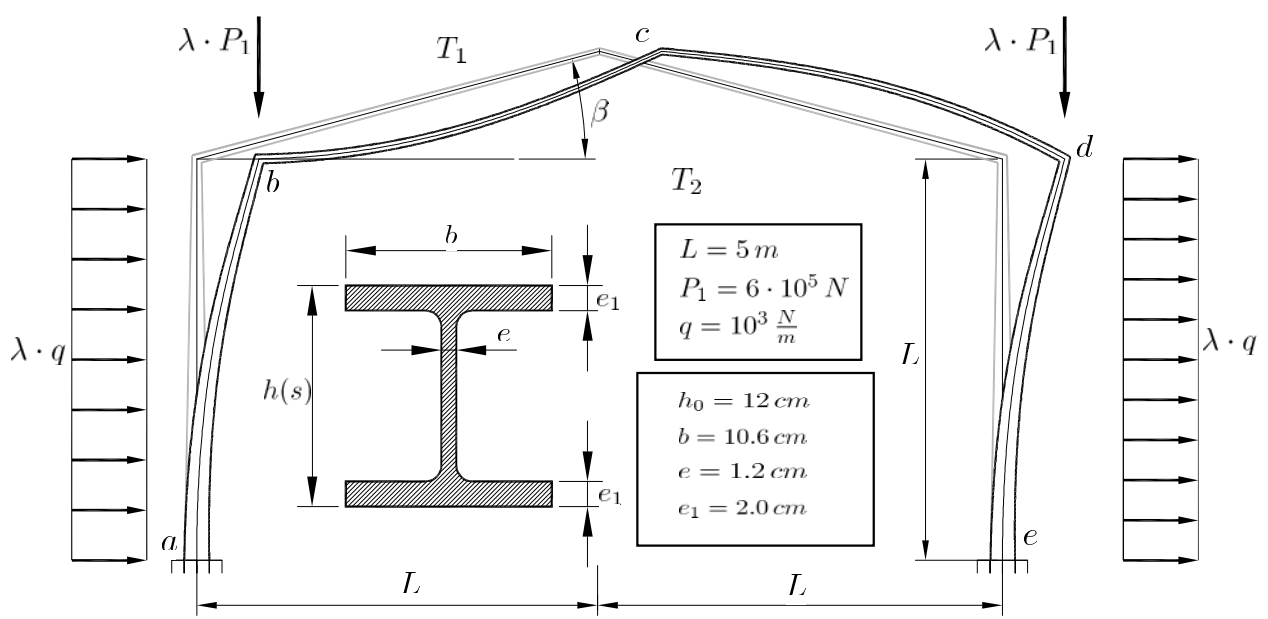

Figura 6.- Pórtico simple a dos aguas, resultados y modo de pandeo. 


\begin{tabular}{|ll|ll|}
\hline \multicolumn{2}{|l|}{ perfil uniforme } & \multicolumn{2}{|c|}{ variación lineal del canto } \\
$r_{a}=r_{e}=h_{0}$ & $\lambda_{c r i}=0.939$ & $r_{a}=r_{e}=24.0 \mathrm{~cm} \quad \lambda_{c r i}=1.516$ \\
$r_{b}=r_{d}=h_{0}$ & $\sigma_{e q v}=165.6357 \mathrm{MPa}$ & $r_{b}=r_{d}=9.151 \mathrm{~cm}$ & $\sigma_{e q v}=113.8735 \mathrm{MPa}$ \\
$r_{c}=h_{0}$ & & $r_{c}=5.698 \mathrm{~cm}$ & \\
\hline
\end{tabular}

Tabla 4.- Pórtico simple a dos aguas, resultados.

De nuevo, y a modo de ejemplo, se puede modificar alguna de las condiciones de contorno iniciales del problema y ver cómo afecta al grado de estabilidad de la estructura y al nivel máximo de tensiones, así, suponiendo pórtico triarticulado (en secciones $a, c$ y $e$ ) la solución se modifica y lleva a los resultados indicados en la Tabla 5:

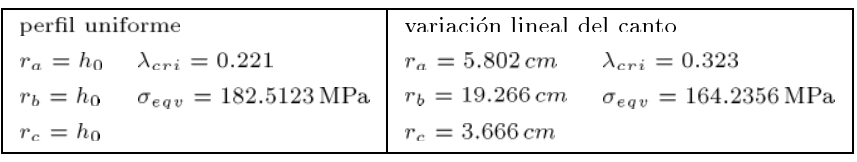

Tabla 5.- Pórtico simple a dos aguas, pilares apoyados y clave en el dintel.

\section{6.- CONCLUSIONES}

En primer lugar, recordar que todo problema de diseño se reduce siempre a un problema de optimización, en general con uno o más objetivos ponderados y con distintos tipos de restricciones: funcionalidad, seguridad, estabilidad, precio, estética, etc., lo que justifica el interés de este tipo de aportaciones y más hoy en día donde se tiende a abordar problemas de multifísica con equipos multidisciplinares.

Respecto a la metodología, el punto de partida ha sido la teoría de pandeo de Euler, que asume pequeños desplazamientos y pequeñas deformaciones junto con el planteamiento del equilibrio en la configuración deformada (Teoría de Segundo Orden), y que da lugar a los conceptos de carga crítica y modo de pandeo, consecuencia del modelo matemático planteado. Se trata de un método de cálculo que no se puede englobar dentro de los métodos de rigidez (o de equilibrio) ni como un método de flexibilidad (o de compatibilidad), sino que es un método novedoso basado en la formulacion diferencial a nivel de barra y en el cumplimiento riguroso de las condiciones de equilibrio y compatibilidad a nivel de estructura. Este plantemiento tiene la gran ventaja de poder considerar sección transversal variable a lo largo de cada barra sin el requisito de los usuales métodos de cálculo de calcular la matriz de rigidez o flexibilidad, y por lo tanto, sin la necesidad de actualizar dicha matriz para cada barra en cada iteración, lo que supone un gran ahorro de cómputo en el proceso de optimización. Así mismo, también permite considerar en el análisis cualquier tipo de carga, incluido el efecto del peso propio y de la temperatura.

Con el método propuesto, el cálculo de la respuesta de la estructurase hace muy sencillo y directo. Además el planteamiento matemático de la inestabilidad como un problema de autovalores gracias a las ecuaciones de estabilidad junto con las de equilibrio, compatibilidad y condiciones de contorno, permite obtener como resultado adicional del análisis la carga crítica y el modo de pandeo con esa misma metodología.

La herramienta de análisis desarrollada es sistemática, sencilla, y permite incluir otros modelos teóricos (lineal, no-lineal, grandes desplazamientos, etc.), cargas de cualquier tipo dentro del elemento, todo tipo de libertades en la uniones, cualquier tipo de apoyo incluido apoyos elásticos, además de lógicamente barras no prismáticas.

La principal desventaja viene dada por las características típicas de un método de optimización no lineal, sobre todo en lo relativo a la limitación en el número de parámetros para que los tiempos de cómputo no sean excesivos. De hecho en programación no lineal, en la actualidad, existe toda una línea de investigación con el objetivo de desarrollar técnicas numéricas orientadas a dar solución a problemas con gran número de parámetros. Algo que se está consiguiendo gracias al desarrollo de algoritmos cada vez más eficientes y al continuo avance de la capacidad de cálculo. 


\section{7.- Referencias}

[1] Atanackovic T. "Optimal shape of an elastic rod in flexural-torsional buckling". Z. Angew. Math. Mech. Vol.87-6 p.399-405 2007.

[2] Cleve M. "Numerical Computing with MATLAB". SIAM Philadelphia 2004.

[3] Cacho M, Lorenzana A. "Cálculo directo de la carga crítica de pandeo de pórticos. Parte II". Rev. Int. Mét. Num. Cálc. Dis. Ing. Vol.26 p.31-8 2010.

[4] Fu K, Ren D. "Optimization of axially loaded non-prismatic column". Computers and Structures Vol.431 p. $159-621992$.

[5] Gilat A. "MATLAB: An Introduction With Applications". 3a ed. John Wiley \& Sons, Inc. 2008. ISBN: 978-0-470-10877-2.

[6] Hernandez-Vazquez J. "ESTRUCTURAS Y EFECTOS DINAMICOS DEL VIENTO ". DYNA Ingeniería e Industria Vol.80-2 p.09-12 2005

[7] Keller J. "The shape of the strongest column". Arch. Ration. Mech. Anal. Vol.5 p.275-85 1960.

[8] Lorenzana A, Cacho M. "Cálculo directo de la carga crítica de pandeo de pórticos. Parte I". Rev. Int. Mét. Num. Cálc. Dis. Ing. Vol.25-3 p.247-58 2009.

[9] Monleón S. "Análisis de vigas, arcos, placas y láminas: una presentación unificada". Universidad Politécnica de Valencia 1999.

[10] Maciel M. "Introducción a la Optimización Numérica". Ph. D in Mathematical Sciences Rice University Houston Texas 2000.

[11] Miralbes-Buil R, Castejon-Herrer L. "Diseño y optimización de plumines para carretillas manipuladoras". DYNA Ingeniería e Industria Vol.83-5 p.182-190 2009.

[12] Nocedal J, Wright S. "Numerical Optimization". Springer-Verlag New York Inc 1999. ISBN: 0-38798793-2.

[13] Ortega M, Romero J, Rosa E. "Un estudio histórico del problema de las piezas prismáticas rectas sometidas a compresión. Parte I y II". Informes de la Construcción Vol.59, 507 p.69-81 y 508 p.61-71 2007.

[14] Sebastián-Sanz J, Casado-Sanchez C, Lorenzana-Iban A, et al. "Sistemas de control de vibraciones para gruas torre". DYNA Ingeniería e Industria Vol.84-3 p.237-244 2009.

[15] Simitses G, Hodges D. "Fundamentals of Structural Stability". Elsevier Inc. 2006. ISBN: 978-0-75067875-9.

[16] Spillers W, Levy R. "Optimal design for axisymmetric cylindrical shell buckling". J. Engng. Mech. Vol.115 p.1683-90 1989.

[17] Spillers W, Levy R. "Optimal design for plate buckling". J. Struct. Engng. Vol.116 p.850-8 1990.

[18] Tadjbakhsh T, Keller J. "Strongest column and isoparametric inequalities for eigenvalues". J. Appl. Math. Vol.9 p.159-64 1962.

[19] Taylor J. "The strongest column - an energy approach". J. Appl. Mech. Vol.34 p.486-7 1962.

[20] Timoshenko S. "History of Strength of Materials". Dover Publ. Inc. New York 1953.

[21] Timoshenko S, Goodier J. "Teoría de la Elasticidad". Urmo 1975.

[22] Truesdell C. "Ensayos de historia de la Mecánica". Tecnos Madrid Spain 1975. 

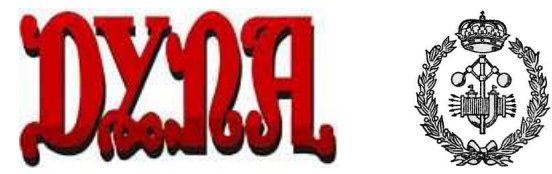

\section{CONFIRMACION DE APROBACION DE ARTICULO PARA SU PUBLICACION EN LA REVISTA DYNA}

El artículo y autor que figura al final de este párrafo, ha sido aprobado para su próxima publicación en la revista DYNA Ingeniería e Industria.

OPTIMIZACIÓN GLOBAL DE PÓRTICOS 2D CON BARRAS DE SECCIÓN VARIABLE MARIANO CACHO PÉREZ

Y para que así conste, a petición del interesado, expedimos el presente certificado.

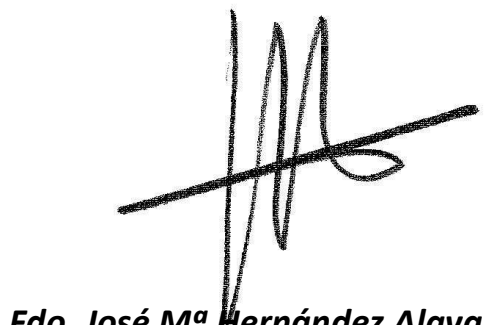

Director

Bilbao,

jueves, 16 de septiembre de 2010 



\section{Bibliografía}

[1] E. Alarcón. Leyes de comportamiento de los materiales. Curso de Especialización Superior y Aplicación Práctica del Método de los Elementos Finitos, 1994.

[2] F. Armero y D. Ehrlich. Finite element methods for the analysis of softening plastic hinges in beams and frames. Comput. Mech., 35:237-264, 2005.

[3] T.M. Atanackovic. Optimal shape of an elastic rod in flexural-torsional buckling. $Z$. Angew. Math. Mech., 87(6):399-405, 2007.

[4] B. Balachandran. Vibraciones. Thomson, México, 2006.

[5] M. Cacho y A. Lorenzana. Cálculo directo de la carga crítica de pandeo de pórticos. Parte II. Rev. Int. Mét. Num. Cálc. Dise. Ing., 26:31-38, 2010.

[6] J. Cañas. Análisis y diseño elastoplástico de estructuras planas formadas por barras prismáticas. PhD thesis, Universidad de Sevilla, 1986.

[7] J. Chakrabarty. Theory of Plasticity. Elsevier, 2006.

[8] E. Chica. Modelo de comportamiento elastoplástico degradable: aplicación al elemento barra 2D. Tesis Doctoral, Dpto. C. A., I.T. y Mecánica de Medios Continuos y Teoría de Estructuras, Universidad de Valladolid, 2009.

[9] B.M. Cleve. Numerical Computing with MATLAB. Society for Industrial and Applied Mathematics, 2004.

[10] Z.Ma. Cohn y A. Franchi. A computer system for structural plasticity. J. Strut. Div. ASCE, 105(4):789-804, 1979.

[11] J.A.T. de Feitas y D. Lloyd Smith. Elastoplastic analysis of planar structures for large displacements. J. Struct. Mech., 12:419-445, 1995.

[12] J.C. del Caño y J.A. Garrido. Elasticidad. Dpto. C.A., I.T. y Mecánica de los Medios Continuos y Teoría de Estructuras, Escuela de Ingenierías Industriales, Universidad de Valladolid, 2002.

[13] M. Doblaré y E. Alarcón. Elementos de plasticidad. 1994. 
[14] R.V. Dukkipati. Vibrations : problem solving companion. Alpha Science International, 2007 .

[15] K.C. Fu y D. Ren. Optimization of axially loaded non-prismatic column. Computers and Structures, 43(1):159-162, 1992.

[16] A. Gilat. MATLAB: An Introduction With Applications. John Willey \& Sons, Inc., 2005.

[17] E. Graff y Eisenberger. Non-linear analysis of framed structures with axial constraints. Computers and Structures, 38:345-351, 1991.

[18] M.R. Horne. Plastic theory of structures. Nelson, London, 1971.

[19] D.J. Inman. Engineering vibration. Pearson Prentice-Hall, New Jersey, 3rd ed., 2009.

[20] W. Jiang. A new constitutive model in the theory of plasticity. Pressure Vessel Thechnol., 117:365-371, 1995.

[21] J.B. Keller. The shape of the strongest column. Arch. Ration. Mech. Anal., 5:275-285, 1960.

[22] S. Kim, M.K. Kim, y W. Chen. Improved refined plastic hinge analysis accounting for strain reversal. Engineering Structures, 20:15-25, 2000.

[23] S. Krenk, C. Vissing-Jörgensenm, y L. Thesbbjerg. Efficient collapse analysis for framed structures. Computers and Structures, 72:481-496, 1999.

[24] A. Lorenzana y M. Cacho. Cálculo directo de la carga crítica de pandeo de pórticos. Parte I. Rev. Int. Mét. Num. Cálc. Dise. Ing., 25(3):247-258, 2009.

[25] A. Lorenzana y J.A. Garrido. Analysis of the elastoplastic problem involving finite plastic strain using the boundary element method. Computers and Structures, 73:147-159, 1999.

[26] J. Lubliner. Plasticity theory. Maxwell Macmillan International Editions, 1990.

[27] M.C. Maciel. Introducción a la Optimización Numérica. PhD in Mathematical Sciences, Rice University, Houston, Texas, 2000.

[28] G. Maier y al. Inelastic analysis of reinforced concrete frames by quadratic programming. Simposium on Inelasticity and Nonlinearities in Strut. Concr., Univ. of Waterloo, Canada, 1973.

[29] G. Maier y G. Cocchetti. Elastic-plastic and limit-state analyses of frames with softening plastic-hinge models by mathematical programming. International Journal of Solids and Structures, 40:7219-7244, 2003.

[30] L. Meirovitch. Elements of vibration analysis. MacGraw-Hill, 2nd ed., New York, 1986. 
[31] O. Möller y M. Rubinstein. Análisis dinámico no lineal físico y geométrico de barras: discusión del campo de aplicación de teorías aproximadas. Revista Internacional de Métodos Numéricos para Cálculo y Diseño en Ingeniería, 11:151-182, 1995.

[32] S. Monleón. Análisis de vigas, arcos, placas y láminas, una presentación unificada. Universidad Politécnica de Valencia, 1999.

[33] R. Muralidhar y J.R. Jagannatha. New models for optimal truss topology in limit design based on unified elastic/plastic analysis. Comput. Methods Appl. Mech. Engrg., 140:109$138,1997$.

[34] B.G. Neal. The plastic methods of structural analysis. Science Paperbacks,London, 3rd edition, 1977.

[35] J. Nocedal y S.J. Wright. Numerical Optimization. Springer Series in Operations Research, 1999.

[36] E. Oñate. Cálculo de estructuras por el Método de Elementos Finitos. Análisis estático lineal. Centro Internacional de Métodos Numéricos en Ingeniería, 1992.

[37] M.A. Ortega, J.L. Romero, y E. Rosa. A historical study of the problem of straight prismatic elements subjected to compression. Informes de la Construcción, 59, 507:69-81, 1997.

[38] D. Reynolds, J. McConnachie, P. Bettess, W.C. Christie, y J.W. Bull. Reverse adaptivity. A new evolutionary tool for structural optimization. International Journal of Numerical Methods in Engineering, 45:529-552, 1999.

[39] J.Y. Richard-Liew, H. Chen, N.E. Shanmugam, y W.F Chen. Improved nonlinear hinge analysis of space frame structures. Engineering Structures, 22:1324-1338, 2000.

[40] J.Y. Richard-Liew, D.W. White, y W.F. Chen. Limit states design of semi-rigid frames using advanced analysis. Part 2: analysis and desing. J. Const. Steel Res., 26:29-57, 1993.

[41] M. Roufaiel y C. Meyer. Analytical modeling of histeretic behavior of R/C frames. $J$. Strut. Div. ASCE, 113(3):429-443, 1987.

[42] M.P. Saka y M.S. Hayalioglu. Optimum design of geometrically nonlinear elastic-plastic steel frames. Computers and Structures, 38(3):329-344, 1991.

[43] G.J. Simitses y D.H. Hodges. Fundamentals of Structural Stability. Elsevier Inc., 2006.

[44] W.R. Spillers y R. Levy. Optimal design for axisymmetric cylindrical shell buckling. J. Engng. Mech., 115:1683-1690, 1989.

[45] W.R. Spillers y R. Levy. Optimal design for plate buckling. J. Struct. Engng., 116:850-858, 1990. 
[46] P. Symonds y B. Neal. The calculation of collapse loads for frame structures. Jour. Instn. Civil Engrs, 35:21-40, 1951.

[47] T. Tadjbakhsh y J.B. Keller. Strongest column and isoparametric inequalities for eigenvalues. J. Appl. Math., 9:159-164, 1962.

[48] T. Takeda y al. Reinforced concrete response to simulated earthquakes. J. Strut. Div. ASCE, 96:2557-2573, 1970.

[49] J.E. Taylor. The strongest column: an energy approach. J. Appl. Mech., 34:486-487, 1962.

[50] J.M.G. Terán. Formulación y análisis del comportamiento elastoplástico acoplado y no lineal geométrico de sistemas estructurales de barras. Tesis Doctoral, Dpto. Resistencia de Materiales y Estructuras, Universidad de Valladolid, 2002.

[51] S.P. Timoshenko. Vibration Problems In Engineering. D. Van Nostrand Company, Inc. New York, 1937.

[52] S.P. Timoshenko. History of Strength of Materials. Dover Publ. Inc., New York, 1953.

[53] S.P. Timoshenko y J.N. Goodier. Teoría de la Elasticidad. Urmo, 1975.

[54] F. Tin-Loi y J.S. Misa. Large displacement elastoplastic analysis of semirigid steel frames. International Journal for Numerical Methods in Engineering, 87:741-762, 1996.

[55] F. Tin-Loi y S. Tangaramvong. Limit analysis of elastoplastic frames considering 2nd-order geometricnonlinearity and displacement constraints. International Journal of Mechanical Sciences, 51:179-191, 2009.

[56] C. Truesdell. Ensayos de Historia de la Mecánica. Tecnos, Madrid, Spain, 1975.

[57] H. Weimin y B.D. Reddy. Plasticity. Mathematical Theory and numerical analysis. Springer, 1999.

[58] D.W. White. Plastic hinge methods for advanced analysis of steel frames. J. Const. Steel Res., 24:121-152, 1993.

[59] M.B. Wong. Plastic frame analysis under fire conditions. J. of Structural Engng., 127-133, 2001.

[60] M.B. Wong y F. Tin-Loi. Analysis of frames involving geometrical and material nonlinearities. Computers Structures, 34:641-646, 1990.

[61] O.C. Zienkiewicz y R.L. Taylor. El método de los elementos finitos. Centro Intern. de Métodos Numéricos en Ingeniería, 1995. 
Anexo I

Trabajos en desarrollo 
Artículos de investigación en proceso de elaboración, pendientes de evaluación y, en su caso, de publicación:

a) AnÁlisis LÍmite De PóRticos Planos INCLUYENDO ESTABILIDAD Y COMPORTAMIENTO VIBRATORIO. PARTE I

M. Cacho y A. Lorenzana

Rev. Int. Mét. Num. Cálc. Dise. Ing.

b) AnÁlisis límite DE PÓRTiCos Planos INCLUYENDO ESTABILIDAD Y COMPORTAMIENTO VIBRATORIO. PARTE II

M. Cacho y A. Lorenzana

Rev. Int. Mét. Num. Cálc. Dise. Ing.

c) INFLUENCIA DE LA PLASTIFICACiÓN EN LA CARGA DE PANDEO DE PÓRTiCOS 2D M. Cacho Pérez y A. Lorenzana Ibán DYNA Ingeniería e Industria. 



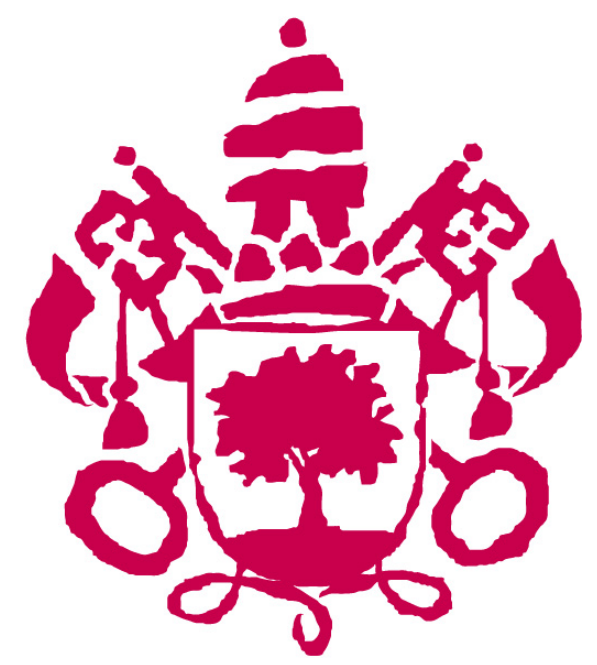

UVa 
